T. Kappeler and R. Montalto (2016) “Canonical Coordinates with Tame Estimates for the Defocusing NLS Equation on the Circle,"

International Mathematics Research Notices, Vol. 2018, No. 5, pp. 1473-1531

Advance Access Publication December 26, 2016

doi: 10.1093/imrn/rnw233

\title{
Canonical Coordinates with Tame Estimates for the Defocusing NLS Equation on the Circle
}

\section{Thomas Kappeler* and Riccardo Montalto}

Institut für Mathematik, Universität Zürich, Winterthurerstrasse 190, CH-8057 Zürich, Switzerland

*Correspondence to be sent to: e-mail: thomas.kappeler@math.uzh.ch

In a case study for integrable PDEs, we construct real analytic, canonical coordinates for the defocusing NLS equation on the circle, specifically tailored to the needs in perturbation theory. They are defined in neighbourhoods of families of finite-dimensional invariant tori and are shown to satisfy together with their derivatives tame estimates. When expressed in these coordinates, the defocusing NLS Hamiltonian is in normal form up to order three.

\section{Introduction}

In form of a case study for integrable PDEs (iPDEs), the goal of this paper is to construct canonical coordinates for the defocusing NLS (dNLS) equation, specifically tailored to the needs in perturbation theory. We consider the dNLS equation in one space dimension

$$
\mathrm{i} \partial_{t} u=-\partial_{x}^{2} u+2|u|^{2} u, \quad x \in \mathbb{T}:=\mathbb{R} / \mathbb{Z}
$$

on the Sobolev space $H_{\mathbb{C}}^{s} \equiv H^{s}(\mathbb{T}, \mathbb{C})$ of complex-valued functions on $\mathbb{T}$, whose distributional derivatives up to order $s \in \mathbb{Z}_{\geq 0}$ are in $L^{2}(\mathbb{T}, \mathbb{C})$. Equation (1.1) can be viewed as a Hamiltonian PDE, obtained by restricting the Hamiltonian system on the phase space

Received July 22, 2016; Revised July 22, 2016; Accepted September 22, 2016

Communicated by Prof. Jonatan Lenells

(C) The Author(s) 2016. Published by Oxford University Press. All rights reserved. For permissions, please e-mail: journals.permission@oup.com. 
1474 T. Kappeler and R. Montalto

$H_{c}^{s}:=H_{\mathbb{C}}^{s} \times H_{\mathbb{C}}^{s}$ with Poisson bracket and Hamiltonian given by

$$
\{\mathcal{F}, \mathcal{G}\}(u, v)=-\mathrm{i} \int_{0}^{1}\left(\partial_{u} \mathcal{F} \partial_{V} \mathcal{G}-\partial_{V} \mathcal{F} \partial_{u} \mathcal{G}\right) \mathrm{d} x, \quad \mathcal{H}^{\mathrm{nls}}(u, v)=\int_{0}^{1}\left(\partial_{x} u \partial_{x} v+u^{2} v^{2}\right) \mathrm{d} x
$$

to the real subspace $H_{r}^{s}$ of $H_{c}^{s}$ consisting of elements $(u, v)$ with $v=\bar{u}$. Here $\mathcal{F}, \mathcal{G}$ are $\mathcal{C}^{1}$-smooth complex-valued functionals on $H_{c}^{s}$ with sufficiently regular $L^{2}$-gradients. Equation (1.1) can then be rewritten as $\partial_{t} u=-\left.\mathrm{i} \partial_{V} \mathcal{H}^{\mathrm{nls}}\right|_{V=\bar{u}}$. The dNLS equation is an iPDE and according to [10], admits global Birkhoff coordinates on $H_{\mathbb{C}}^{s}$ with $s \in \mathbb{Z}_{\geq 0}$. To state the main results of this paper we first need to describe these coordinates in more detail: for any $s \in \mathbb{Z}_{\geq 0}$, let

$$
\begin{gathered}
h_{\mathbb{C}}^{s} \equiv h^{s}(\mathbb{Z}, \mathbb{C}):=\left\{x=\left(x_{n}\right)_{n \in \mathbb{Z}} \subseteq \mathbb{C}:\|x\|_{s}<+\infty\right\},\|x\|_{s}:=\sum_{n \in \mathbb{Z}}\langle n\rangle^{2 s}\left|x_{n}\right|^{2},\langle n\rangle:=\max \{1,|n|\}, \\
h^{s} \equiv h^{s}(\mathbb{Z}, \mathbb{R}):=\left\{\left(x_{n}\right)_{n \in \mathbb{Z}} \in h_{\mathbb{C}}^{s}: x_{n} \in \mathbb{R} \forall n \in \mathbb{Z}\right\}
\end{gathered}
$$

and

$$
h_{c}^{s}:=h_{\mathbb{C}}^{s} \times h_{\mathbb{C}^{\prime}}^{s} \quad h_{r}^{s}:=h^{s} \times h^{s}
$$

The Sobolev space $H_{\mathbb{C}}^{s}$ can then be described by

$$
H_{\mathbb{C}}^{s}=\left\{u=\sum_{n \in \mathbb{Z}} u_{n} \mathrm{e}^{2 \pi \mathrm{i} n x}:\left(u_{n}\right)_{n \in \mathbb{Z}} \in h_{\mathbb{C}}^{s}\right\}, \quad\|u\|_{s}:=\left\|\left(u_{n}\right)_{n \in \mathbb{Z}}\right\|_{s} .
$$

Furthermore let

$$
\begin{aligned}
\ell^{1,2} \equiv \ell^{1,2}(\mathbb{Z}, \mathbb{R}) & :=\left\{x=\left(x_{n}\right)_{n \in \mathbb{Z}} \subset \mathbb{R}:\|x\|_{1,2}:=\sum_{n \in \mathbb{Z}}\langle n\rangle^{2}\left|x_{n}\right|<+\infty\right\}, \\
\ell_{+}^{1,2} & :=\left\{\left(x_{n}\right)_{n \in \mathbb{Z}} \in \ell^{1,2}: \quad x_{n} \geq 0, \quad \forall n \in \mathbb{Z}\right\}
\end{aligned}
$$

and define the following version $F_{\text {nls }}$ of the Fourier transform, introduced in [10],

$$
F_{\mathrm{nls}}: H_{c}^{0} \rightarrow h_{c^{\prime}}^{0} \quad(u, v) \mapsto\left(-\frac{1}{\sqrt{2}}\left(u_{-n}+v_{n}\right),-\frac{\mathrm{i}}{\sqrt{2}}\left(u_{-n}-v_{n}\right)\right),
$$

where $u_{n}$ denotes the $n$th Fourier coefficient of $u, u_{n}:=\int_{0}^{1} u(x) \mathrm{e}^{-2 \pi \mathrm{in} x} \mathrm{~d} x$. Note that for $v=\bar{u}$, one has $v_{n}=\bar{u}_{-n}$ for any $n \in \mathbb{Z}$, implying that

$$
F_{\mathrm{nls}}(u, \bar{u})=\left(-\sqrt{2} \operatorname{Re}\left(u_{-n}\right), \sqrt{2} \operatorname{Im}\left(u_{-n}\right)\right)
$$


The inverse of $F_{\mathrm{nls}}$ is then given by

$F_{\text {nls }}^{-1}: h_{c}^{0} \rightarrow H_{c}^{0}, \quad\left(\left(x_{n}\right)_{n \in \mathbb{Z}},\left(y_{n}\right)_{n \in \mathbb{Z}}\right) \mapsto\left(-\frac{1}{\sqrt{2}} \sum_{n \in \mathbb{Z}}\left(x_{-n}-\mathrm{i} y_{-n}\right) \mathrm{e}^{2 \pi \mathrm{i} n x},-\frac{1}{\sqrt{2}} \sum_{n \in \mathbb{Z}}\left(x_{n}+\mathrm{i} y_{n}\right) \mathrm{e}^{2 \pi \mathrm{i} n x}\right)$.

Finally we recall that a possibly nonlinear map $F: U \rightarrow Y$ of a subset $U$ of a Banach space $X$ into another Banach space $Y$ is said to be bounded if $F(V)$ is bounded for any bounded subset $V$ in $U$.

Theorem $1.1([10,14])$. There exists a neighbourhood $\mathcal{W}$ of $H_{r}^{0}$ in $H_{c}^{0}$ and an analytic map

$$
\Phi^{\mathrm{nls}}: \mathcal{W} \rightarrow h_{c^{\prime}}^{0} \quad(u, v) \mapsto\left(\left(x_{n}\right)_{n \in \mathbb{Z}},\left(y_{n}\right)_{n \in \mathbb{Z}}\right)
$$

with $\Phi^{\text {nls }}(0)=0$ such that the following holds:

(B1) For any $s \in \mathbb{Z}_{\geq 0}, \Phi^{\mathrm{nls}}\left(H_{r}^{s}\right) \subseteq h_{r}^{s}$ and $\Phi^{\mathrm{nls}}: H_{r}^{s} \rightarrow h_{r}^{s}$ is a real analytic diffeomorphism.

(B2) The map $\Phi^{\text {nls }}$ is canonical, meaning that on $\mathcal{W},\left\{x_{n}, y_{n}\right\}=-1$ and all the other brackets between coordinate functions vanish.

(B3) The Hamiltonian $H^{\text {nls }}:=\mathcal{H}^{\text {nls }} \circ\left(\Phi^{\text {nls }}\right)^{-1}$, defined on $h_{r}^{1}$, is a function of the actions $I_{n}:=\left(x_{n}^{2}+y_{n}^{2}\right) / 2, n \in \mathbb{Z}$, only and $H^{\text {nls }}: \ell_{+}^{1,2} \rightarrow \mathbb{R}, I \mapsto H^{\text {nls }}(I)$ is real analytic.

(B4) The differential $d_{0} \Phi^{\text {nls }}$ of $\Phi^{\text {nls }}$ at 0 is the Fourier transform $F_{\text {nls }}$ defined in (1.3).

(B5) The nonlinear parts $A^{\text {nls }}:=\Phi^{\text {nls }}-F_{\text {nls }}$ of $\Phi^{\text {nls }}$ and $B^{\text {nls }}:=\Psi^{\text {nls }}-F_{\text {nls }}^{-1}$ of $\Psi^{\text {nls }}:=$ $\left(\Phi^{\text {nls }}\right)^{-1}$ are one smoothing, meaning that for any $s \in \mathbb{Z}_{\geq 1}$,

$$
A^{\mathrm{nls}}: H_{r}^{s} \rightarrow h_{r}^{s+1} \quad \text { and } \quad B^{\mathrm{nls}}: h_{r}^{s} \rightarrow H_{r}^{s+1}
$$

are real analytic and bounded.

The maps $\Phi^{\text {nls }}, \Psi^{\text {nls }}$ are referred to as Birkhoff maps and the coordinates $\left(\left(x_{n}, Y_{n}\right)\right)_{n \in \mathbb{Z}}$ as Birkhoff coordinates for the dNLS equation.

Birkhoff coordinates are a tool to study perturbations of the dNLS equation far away from the equilibrium. In particular, in [2] they were used to show the existence of finite-dimensional invariant tori of large size for Hamiltonian perturbations of this equation, involving no derivatives of $u$. So far, no such results have been obtained for 
perturbations involving $\partial_{x} u$ (and possibly $\partial_{x}^{2} u$ ) - see $[2,4-7,9,12,17]$ for results on perturbations of the dNLS equation on the circle obtained so far. In view of the recent results in [1] concerning the existence of small quasi-periodic solutions of quasi-linear Hamiltonian perturbations of the Karteweg de Vries (KdV) equation and our results in [2] described above, we expect that Hamiltonian perturbations of the dNLS equation, involving $\partial_{x} u$ (and possibly $\partial_{x}^{2} u$ ), also admit large quasi-periodic solutions, also referred to as multi-solitons. For this purpose, the scheme developed in [2] has to be considerably refined. In particular, canonical coordinates are needed which together with their derivatives satisfy tame estimates. In [19], such estimates were derived for $\Phi^{\text {nls }}: H_{r}^{0} \rightarrow h_{r}^{0}$ on the real subspaces $H_{r}^{s}$ and for its inverse $\Psi^{\text {nls }}: h_{r}^{0} \rightarrow H_{r}^{0}$ on the real subspaces $h_{r}^{s}$ where $s \in \mathbb{Z}_{s \geq 2}$. But so far they are not available for their derivatives. In this paper, we prove how to use the Birkhoff coordinates to construct near bounded, integrable, finite-dimensional subsystems (iSS) of the dNLS equation, local canonical coordinates so that they satisfy tame estimates and the dNLS Hamiltonian, when expressed in these coordinates, is in normal form up to order three-see Theorem 1.2 for a precise statement. In future work, we will use these coordinates as a starting point for applying a KAM scheme to reduce certain linear operators with tame estimates, which come up in the Nash Moser iteration, to operators with constant coefficients. Recently, such schemes have been further developed in significant ways. In the context of the dNLS equation, results of this type in [3] will be particularly relevant.

To state our main result, we need to introduce some more notation. For any $S \subseteq \mathbb{Z}$ with $|S|<+\infty$, let $S^{\perp}:=\mathbb{Z} \backslash S$. By a slight abuse of notation, we identify $h_{c}^{s}$ with $\mathbb{C}^{S} \times \mathbb{C}^{S} \times h_{\perp c}^{s}$ and $h_{r}^{s}$ with $\mathbb{R}^{S} \times \mathbb{R}^{S} \times h_{\perp r}^{s}$ where

$$
h_{\perp C}^{s}:=h^{s}\left(S^{\perp}, \mathbb{C}\right) \times h^{s}\left(S^{\perp}, \mathbb{C}\right), \quad h_{\perp r}^{s}:=h^{s}\left(S^{\perp}, \mathbb{R}\right) \times h^{s}\left(S^{\perp}, \mathbb{R}\right)
$$

Accordingly, an element $z \in h_{c}^{0}$ is written as

$$
z=\left(z_{S}, z_{\perp}\right), \quad z_{S}=\left(\left(x_{j}\right)_{j \in S},\left(y_{j}\right)_{j \in S}\right), \quad z_{\perp}=\left(\left(x_{j}\right)_{j \in S^{\perp}},\left(y_{j}\right)_{j \in S^{\perp}}\right),
$$

and as norm we choose $\|z\|_{S}:=\left\|z_{S}\right\|+\left\|z_{\perp}\right\|_{S}$ where

$$
\left\|z_{S}\right\| \equiv\left\|z_{S}\right\|_{0}:=\left(\sum_{j \in S}\left|x_{j}\right|^{2}+\left|y_{j}\right|^{2}\right)^{\frac{1}{2}}, \quad\left\|z_{\perp}\right\|_{s}:=\left(\sum_{j \in S^{\perp}}\langle j\rangle^{2 s}\left(\left|x_{j}\right|^{2}+\left|y_{j}\right|^{2}\right)\right)^{\frac{1}{2}} .
$$

Furthermore, we introduce the bilinear form

$$
\left(z_{\perp}, z_{\perp}^{\prime}\right)_{r}:=\sum_{j \in S^{\perp}} x_{j} x_{j}^{\prime}+y_{j} y_{j}^{\prime}, \quad z_{\perp}=\left(x_{\perp}, y_{\perp}\right), z_{\perp}^{\prime}=\left(x_{\perp}^{\prime}, y_{\perp}^{\prime}\right) \in h_{\perp c}
$$


and write the sequence of actions $I=\left(I_{k}\right)_{k \in \mathbb{Z}}$ as $\left(I_{S}, I_{\perp}\right)$ where

$$
I_{S}:=\left(I_{k}\right)_{k \in S}, \quad I_{\perp}:=\left(I_{k}\right)_{k \in S^{\perp}}, \quad I_{k} \equiv I_{k}(z)=\frac{\left|z_{k}\right|^{2}}{2}=\frac{x_{k}^{2}+y_{k}^{2}}{2}, \quad \forall k \in \mathbb{Z} .
$$

Finally, we introduce the dNLS frequencies

$$
\omega_{k}^{\mathrm{nls}}(I):=\partial_{I_{k}} H^{\mathrm{nls}}(I), \quad k \in \mathbb{Z}
$$

They satisfy asymptotics of the form $\omega_{k}(I)=4 k^{2} \pi^{2}+O(1)$ as $k \rightarrow \pm \infty$. More precisely, the map

$$
\ell_{+}^{1,2} \rightarrow \ell^{\infty},\left(I_{k}\right)_{k \in \mathbb{Z}} \mapsto\left(\omega_{n}^{\mathrm{nls}}(I)-4 \pi^{2} n^{2}\right)_{n \in \mathbb{Z}}
$$

is real analytic and bounded-see Proposition 5.3 in Section 5.2. The main result of this paper is the following one.

Theorem 1.2. Let $S \subseteq \mathbb{Z}$ be finite. For any compact subset $\mathcal{K} \subseteq \mathbb{R}^{S} \times \mathbb{R}^{S}$, there exists an open, bounded, complex neighbourhood $\mathcal{V} \subseteq h_{c}^{0}$ of $\mathcal{K} \times\{0\}$ and a bounded analytic map

$$
\Psi: \mathcal{V} \rightarrow H_{c}^{0}, \quad\left(z_{n}\right)_{n \in \mathbb{Z}} \mapsto W
$$

so that the following holds:

(C1) For any $s \in \mathbb{Z}_{\geq 0}, \Psi\left(\mathcal{V} \cap h_{r}^{s}\right) \subseteq H_{r}^{s}$ and $\Psi: \mathcal{V} \cap h_{r}^{s} \rightarrow H_{r}^{s}$ is a real analytic diffeomorphism onto its image.

(C2) $\Psi$ is canonical, meaning that on $\Psi\left(\mathcal{V} \cap h_{r}^{0}\right),\left\{x_{n}, y_{n}\right\}=-1$ for any $n \in \mathbb{Z}$, whereas all the other brackets between coordinate functions vanish.

(C3) The transformation $\Psi$ is related to $\Psi^{\mathrm{nls}}=\left(\Phi^{\mathrm{nls}}\right)^{-1}$ by

$$
\left.\Psi\right|_{\mathcal{K} \times\{0\}}=\left.\Psi^{\mathrm{nls}}\right|_{\mathcal{K} \times\{0\}}, \quad \mathrm{d} \Psi(z)=\mathrm{d} \Psi^{\mathrm{nls}}(z), \quad \forall z \in \mathcal{K} \times\{0\} .
$$

(C4) The Hamiltonian $\mathcal{H}:=\mathcal{H}^{\text {nls }} \circ \Psi$, defined on $\mathcal{V} \cap h_{r}^{1}$, is in normal form up to order three. More precisely,

$$
\mathcal{H}(z)=H^{\mathrm{nls}}\left(I_{S}, 0\right)+\sum_{n \in S^{\perp}} \omega_{n}^{\mathrm{nls}}\left(I_{S}, 0\right) I_{n}(z)+\mathcal{P}_{3}(z),
$$

where the Hamiltonian $\mathcal{P}_{3}: \mathcal{V} \cap h_{r}^{0} \rightarrow \mathbb{R}$ is real analytic. Furthermore, $\mathcal{P}_{3}$ satisfies the following tame estimates: for any $s \in \mathbb{Z}_{\geq 0}, z \in \mathcal{V} \cap h_{r}^{s}, \widehat{z} \in h_{c^{\prime}}^{s}$

$$
\left\|\nabla \mathcal{P}_{3}(z)\right\|_{s} \lesssim_{s}\left\|z_{\perp}\right\|_{s}\left\|z_{\perp}\right\|_{0}, \quad\left\|d \nabla \mathcal{P}_{3}(z)[\widehat{z}]\right\|_{s} \lesssim_{s}\left\|z_{\perp}\right\|_{s}\|\widehat{Z}\|_{0}+\left\|z_{\perp}\right\|_{0}\|\widehat{Z}\|_{s}
$$


and for any $k \in \mathbb{Z}_{\geq 2}, \widehat{z}_{1}, \ldots, \widehat{z}_{k} \in h_{c^{\prime}}^{s}$

$$
\left\|d^{k} \nabla \mathcal{P}_{3}(z)\left[\widehat{z}_{1}, \ldots, \widehat{z}_{k}\right]\right\|_{s} \lesssim s \sum_{j=1}^{k}\left\|\widehat{z}_{j}\right\|_{s} \prod_{i \neq j}\left\|\widehat{\boldsymbol{z}}_{i}\right\|_{0}+\left\|\boldsymbol{z}_{\perp}\right\|_{s} \prod_{j=1}^{k}\left\|\widehat{\boldsymbol{z}}_{j}\right\|_{0}
$$

Here, the meaning of $\lesssim_{s}$ is the standard one. So, for example, $\left\|\nabla \mathcal{P}_{3}(z)\right\|_{s} \lesssim_{s}$ $\left\|z_{\perp}\right\|_{s}\left\|z_{\perp}\right\|_{0}$ says that there exists a constant $C \equiv C(s)>0$ so that

$$
\left\|\nabla \mathcal{P}_{3}(z)\right\|_{s} \leq C\left\|z_{\perp}\right\|_{s}\left\|z_{\perp}\right\|_{0}, \quad \forall z \in \mathcal{V} \cap h_{r}^{s}
$$

(C5) The nonlinear maps $B:=\Psi-F_{\mathrm{nls}}^{-1}: \mathcal{V} \cap h_{r}^{0} \rightarrow H_{r}^{0}$ and $A:=\Psi^{-1}-F_{\mathrm{nls}}$ : $\Psi(\mathcal{V}) \cap H_{r}^{0} \rightarrow h_{r}^{0}$ are real analytic maps and so is

$$
\mathcal{A}: \mathcal{V} \cap h_{r}^{0} \rightarrow \mathcal{L}\left(H_{c}^{0}, h_{c}^{0}\right), z \mapsto \mathcal{A}(z):=\mathrm{d} \Psi(z)^{-1}-F_{\mathrm{nls}}
$$

On $\mathcal{V} \cap h_{r}^{0}$, the maps $B$ and $\mathcal{A}$ satisfy the following estimates: for any $z \in \mathcal{V} \cap h_{r}^{0}$, $k \in \mathbb{Z}_{\geq 1}, \widehat{z}_{1}, \ldots, \widehat{z}_{k} \in h_{c}^{0}$, and $\widehat{w} \in H_{c}^{0}$ ，

$$
\begin{gathered}
\|B(z)\|_{0} \lesssim 1, \quad\left\|d^{k} B(z)\left[\widehat{z}_{1}, \ldots, \widehat{z}_{k}\right]\right\|_{0} \lesssim_{k} \prod_{j=1}^{k}\left\|\widehat{z}_{j}\right\|_{0} \\
\|\mathcal{A}(z)[\widehat{w}]\|_{0} \lesssim\|\widehat{W}\|_{0}, \quad\left\|d^{k}(\mathcal{A}(z)[\widehat{w}])\left[\widehat{z}_{1}, \ldots, \widehat{z}_{k}\right]\right\|_{0} \lesssim_{k}\|\widehat{w}\|_{0} \prod_{j=1}^{k}\left\|\widehat{z}_{j}\right\|_{0} .
\end{gathered}
$$

Furthermore, $B$ is one smoothing, meaning that for any $s \in \mathbb{Z}_{\geq 1}, B: \mathcal{V} \cap h_{r}^{s} \rightarrow$ $H_{r}^{s+1}$ is real analytic, and satisfies the following tame estimates: for any $k \in \mathbb{Z}_{\geq 1}, z \in \mathcal{V} \cap h_{r}^{s}$, and $\widehat{z}_{1}, \ldots, \widehat{z}_{k} \in h_{c^{\prime}}^{s}$

$$
\begin{aligned}
& \|B(z)\|_{s+1} \lesssim_{s} 1+\left\|z_{\perp}\right\|_{s} \\
& \left\|d^{k} B(z)\left[\widehat{z}_{1}, \ldots, \widehat{z}_{k}\right]\right\|_{s+1} \lesssim_{s, k} \sum_{j=1}^{k}\left\|\widehat{z}_{j}\right\|_{s} \prod_{i \neq j}\left\|\widehat{z}_{i}\right\|_{0}+\left\|z_{\perp}\right\|_{s} \prod_{j=1}^{k}\left\|\widehat{z}_{j}\right\|_{0} .
\end{aligned}
$$

Similarly, the maps $A$ and $\mathcal{A}$ are one smoothing, meaning that for any $s \in \mathbb{Z}_{\geq 1}$, $A: \Psi\left(\mathcal{V} \cap h_{r}^{s}\right) \rightarrow h_{r}^{s+1}$ and $\mathcal{A}: \mathcal{V} \cap h_{r}^{s} \rightarrow \mathcal{L}\left(H_{c}^{s}, h_{c}^{s+1}\right)$ are real analytic. Moreover, $\mathcal{A}$ satisfies the following tame estimates: for any $z \in \mathcal{V} \cap h_{r}^{s}, \widehat{w} \in H_{c}^{s}$,

$$
\|\mathcal{A}(z)[\widehat{W}]\|_{s+1} \lesssim_{s}\left\|z_{\perp}\right\|_{s}\|\widehat{W}\|_{0}+\|\widehat{W}\|_{s}
$$


and for any $k \in \mathbb{Z}_{\geq 1}, \widehat{z}_{1}, \ldots, \widehat{z}_{k} \in h_{c^{\prime}}^{s}$

$$
\begin{gathered}
\left\|d^{k}(\mathcal{A}(z)[\widehat{W}])\left[\widehat{z}_{1}, \ldots, \widehat{z}_{k}\right]\right\|_{s+1} \lesssim_{s, k}\left(\left\|z_{\perp}\right\|_{s}\|\widehat{W}\|_{0}+\|\widehat{W}\|_{s}\right) \prod_{j=1}^{k}\left\|\widehat{z}_{j}\right\|_{0} \\
+\|\widehat{W}\|_{0} \sum_{j=1}^{k}\left\|\widehat{z}_{j}\right\|_{s} \prod_{i \neq j}\left\|\widehat{z}_{i}\right\|_{0} .
\end{gathered}
$$

Remark 1.1. In Theorem 1.2, apart from being compact, no further assumptions on $\mathcal{K}$ are being made. In particular, $\mathcal{K}$ may contain the equilibrium point 0 in which case $\mathcal{K}$ does not admit action-angle coordinates. In subsequent work, the estimates for $\mathcal{A}(z)=$ $\mathrm{d} \Psi(z)^{-1}-F_{\text {nls }}$ will be used to study perturbations of the dNLS equation. Since such estimates are not needed for $A(\Psi(z))$, we have not included them in Theorem 1.2.

Remark 1.2. In Section 7 we present additional results about the map $\Psi$. In particular we study the restrictions of $\Psi$ to $\mathcal{V} \cap h_{r, 1}^{0}$ and $\mathcal{V} \cap h_{r, 2}^{0}$ where $h_{r, 1}^{0}$ and $h_{r, 2}^{0}$ are the dNLS invariant subspaces, corresponding via the Birkhoff map $\Phi^{\text {nls }}$ to potentials $\varphi \in H_{r}^{0}$ which are even and, respectively, odd.

Outline of the construction of $\Psi$ : Let $\mathcal{V}$ be of the form $\mathcal{V}=\mathcal{V}_{S} \times \mathcal{V}_{\perp} \subset h_{c}^{0}$ where $\mathcal{V}_{S}$ is a bounded, open neighbourhood of $\mathcal{K}$ in $\mathbb{C}^{S} \times \mathbb{C}^{S}$ and $\mathcal{V}_{\perp}$ an open ball in $h_{\perp C^{\prime}}^{0}$ centred at $\{0\}$. By Theorem 1.1, $\mathcal{V}_{S}$ and $\mathcal{V}_{\perp}$ can be chosen so that the Birkhoff map $\Psi^{\text {nls }}$ is defined on $\mathcal{V}$ and all the estimates of $\Psi^{\text {nls }}$ and its derivatives used in the sequel are uniform on $\mathcal{V}$. The canonical map $\Psi$ is then defined to be the composition $\Psi:=\Psi_{L} \circ \Psi_{C}$ where $\Psi_{L}$ is the Taylor expansion of $\Psi^{\text {nls }}$ of order one in the normal directions $z_{\perp}$ around $\left(z_{s}, 0\right)$,

$$
\Psi_{L}\left(z_{S}, z_{\perp}\right):=\Psi^{\mathrm{nls}}\left(z_{S}, 0\right)+\mathrm{d} \Psi^{\mathrm{nls}}\left(z_{S}, 0\right)\left[0, z_{\perp}\right],
$$

and $\Psi_{C}$, referred to as symplectic corrector, is chosen so that $\Psi_{L} \circ \Psi_{C}$ becomes symplectic and satisfies the claimed tame estimates.

In his pioneering work [16], Kuksin presents a general scheme for proving KAM-type theorems for semilinear Hamiltonian perturbations of iPDEs in one space dimension, such as the KdV or the sine Gordon (sG) equations, which possess a Lax pair formulation and admit finite-dimensional integrable subsystems, foliated by invariant tori. One of the key elements of his work is a normal form theory for such PDEs. Expanding on work of Krichever [15], Kuksin considers bounded iSS of such an iPDE which admit action-angle coordinates. In the case of the $\mathrm{KdV}$ and the $\mathrm{sG}$ equations, the angle variables are given by 
the celebrated Its Matveev formulas. These action-angle coordinates are complemented by infinitely many coordinates whose construction is based on a set of time periodic solutions, referred to as Floquet solutions, of the PDE obtained by linearizing iPDE along solutions in iSS. The resulting coordinate transformation, denoted in [16] by $\Phi$, is typically not symplectic and to obtain canonical coordinates, an additional coordinate transformation needs to be applied. In [16], Kuksin constructs such a transformation, which he denotes by $\phi$, using arguments of Moser and Weinstein in the given infinite dimensional setup-see [16, Lemma 1.4 and Section 7.1]. To construct the map $\Psi_{C}$ we follow the same scheme of proof. Actually, the following result holds.

Theorem 1.3. Assume that in addition to the assumptions made in Theorem 1.2, the set $\mathcal{K}$ is contained in $(\mathbb{R} \backslash 0)^{S} \times(\mathbb{R} \backslash 0)^{S}$. Then, up to normalizations and natural identifications, $\Psi_{L}$ coincides with the map $\Phi$, obtained by applying the scheme of construction in [16] to the dNLS equation. As a consequence, so does $\Psi=\Psi_{L} \circ \Psi_{C}$ with $\Phi \circ \phi$.

Since Birkhoff coordinates provide a concise, self-contained, and efficient framework for proving Theorem 1.2-in particular the claimed tame estimates, the main goal of our study-Theorem 1.3 also provides in the case of the dNLS equation a valuable alternative for proving the normal form result for this equation, obtained by applying the scheme of proof in [16]. Note also that the assumptions on $\mathcal{K}$ in Theorem 1.2 are slightly weaker than the ones made in the setup of [16].

Organization: The maps $\Psi_{L}$ and $\Psi_{C}$ are introduced and studied in Sections 3 and 4, respectively, after a short Section 2, describing the Hamiltonian setup. In Section 5, we prove Theorem 1.2: in Section 5.1, we show that the composition $\Psi=\Psi_{L} \circ \Psi_{C}$ satisfies the analytic properties, stated in Theorem 1.2, and in the subsequent Section 5.2, the expansion of the dNLS Hamiltonian in the new coordinates is computed up to order three. In Section 5.3 we summarize the proof of Theorem 1.2. Finally, in Section 6 we prove Theorem 1.3 and in Section 7 results, concerning the restriction of $\Psi$ to subsets, satisfying symmetry conditions. In Appendix 1, we recall an infinite-dimensional version of the Poincaré Lemma, needed in Section 4 (cf. from [16], [18]).

Notation: For any $C^{1}$ map $F: h_{c}^{0} \rightarrow X$ with $X$ being a Banach space, we denote by $d_{\perp} F(z)$ the differential of $F$ at $z$ with respect to the variable $z_{\perp}$,

$$
d_{\perp} F(z)\left[\widehat{z}_{\perp}\right]=\sum_{j \in S^{\perp}} \widehat{x}_{j} \partial_{x_{j}} F(z)+\widehat{Y}_{j} \partial_{Y_{j}} F(z), \quad \widehat{z}_{\perp}:=\left(\left(\widehat{x}_{j}\right)_{j \in S^{\perp}},\left(\widehat{y}_{j}\right)_{j \in S^{\perp}}\right) \in h_{\perp C^{\prime}}^{0}
$$


where for any $j \in S^{\perp}, \partial_{X_{j}} F, \partial_{Y_{j}} F \in X$ denote the partial derivatives of $F$ with respect to the variables $x_{j}$ respectively $y_{j}$. Similarly, we define the gradient with respect to the variable $z_{\perp}$ as

$$
\nabla_{\perp} F:=\left(\left(\partial_{X_{j}} F\right)_{j \in S^{\perp}},\left(\partial_{Y_{j}} F\right)_{j \in S^{\perp}}\right)
$$

The gradient of $F$ with respect to $z_{S}$ is denoted by

$$
\nabla_{S} F:=\left(\left(\partial_{x_{j}} F\right)_{j \in S},\left(\partial_{y_{j}} F\right)_{j \in S}\right)
$$

and the differential of $F$ at $z$ with respect to $z_{S}$ by $d_{S} F(z)$,

$$
d_{S} F(z)\left[\widehat{z}_{S}\right]=\sum_{j \in S} \widehat{x}_{j} \partial_{x_{j}} F(z)+\widehat{Y}_{j} \partial_{Y_{j}} F(z), \quad \widehat{z}_{S}:=\left(\left(\widehat{x}_{j}\right)_{j \in S},\left(\widehat{Y}_{j}\right)_{j \in S}\right) \in \mathbb{C}^{S} \times \mathbb{C}^{S}
$$

For the partial derivatives of $F$ with respect to $z_{j}, j \in S$, we use the multi-index notation and write for any $\alpha, \beta \in \mathbb{Z}_{\geq 0}^{S}$

$$
\partial_{S}^{\alpha, \beta} F:=\left(\prod_{j \in S} \partial_{X_{j}}^{\alpha_{j}} \partial_{Y_{j}}^{\beta_{j}}\right) F .
$$

If not stated otherwise, $\mathcal{K}$ denotes a compact subset of $\mathbb{R}^{S} \times \mathbb{R}^{S}$ and $\mathcal{V}$ an open, bounded neighbourhood of $\mathcal{K} \times\{0\}$ in $h_{c}^{0}$ of the form $\mathcal{V}_{S} \times \mathcal{V}_{\perp}$ where $\mathcal{V}_{\perp}$ is a ball in $h_{\perp c}$, centred at 0 We write $\mathcal{V}_{\perp}(\delta)$ to indicate that the radius of the ball $\mathcal{V}_{\perp}$ is $\delta>0$. Finally, we frequently will use the symbols $\lesssim_{s}, \ldots$ to express that a quantity is bounded by another one up to a constant which is "universal," respectively, depends only on the Sobolev index $s$. For example, given two real valued functionals $A, B$ on $\mathcal{V}$ we write $A \lesssim_{s} B$ if there is a constant $C \equiv C(s)$ so that $A(z) \leq C B(z)$ for any $z \in \mathcal{V} \cap h_{r}^{s}$.

\section{Hamiltonian Setup}

In this preliminary section we discuss the Hamiltonian setup, introduced in Section 1 , in more detail and introduce some additional notations.

The Hamiltonian vector field associated with a sufficiently smooth functional $\mathcal{F}: H_{c}^{0} \rightarrow$ $\mathbb{C}$ and the Poisson bracket (1.2) on $H_{c}^{0}$ is denoted by

$$
X_{\mathcal{F}}=\mathrm{i} \rrbracket \nabla \mathcal{F}, \quad \nabla \mathcal{F}:=\left(\nabla_{u} \mathcal{F}, \nabla_{v} \mathcal{F}\right),
$$


1482 T. Kappeler and R. Montalto

where $\nabla_{u} \mathcal{F}, \nabla_{v} \mathcal{F}$ denote the $L^{2}$ gradients with respect to $u$ and $v$, namely

$$
\begin{gathered}
\mathrm{d} \mathcal{F}[(\widehat{u}, 0)]=\int_{\mathbb{T}} \nabla_{u} \mathcal{F} \widehat{u} \mathrm{~d} x, \quad \mathrm{~d} \mathcal{F}[(0, \widehat{v})]=\int_{\mathbb{T}} \nabla_{V} \mathcal{F} \widehat{V} \mathrm{~d} x, \\
\mathbb{J}:=\left(\begin{array}{cc}
0 & -\mathrm{Id} \\
\mathrm{Id} & 0
\end{array}\right): H_{c}^{0} \rightarrow H_{c}^{0}
\end{gathered}
$$

and Id $: H_{\mathbb{C}}^{0} \rightarrow H_{\mathbb{C}}^{0}$ is the identity operator. Furthermore we introduce the non-degenerate bilinear form

$$
\langle\cdot, \cdot\rangle_{r}: H_{c}^{0} \times H_{c}^{0} \rightarrow \mathbb{C}
$$

defined for any $w=(u, v), w^{\prime}=\left(u^{\prime}, v^{\prime}\right) \in H_{c}^{0}$ by

$$
\left\langle W, w^{\prime}\right\rangle_{r}:=\int_{\mathbb{T}} u(x) u^{\prime}(x) \mathrm{d} x+\int_{\mathbb{T}} v(x) v^{\prime}(x) \mathrm{d} x
$$

The subscript $r$ indicates that in the latter integrals, no complex conjugation appears. The Poisson bracket (1.2) then reads

$$
\{\mathcal{F}, \mathcal{G}\}=\langle\nabla \mathcal{F}, \mathrm{i} \mathbb{J} \nabla \mathcal{G}\rangle_{r}
$$

and the symplectic form, associated with it, is the two form

$$
\begin{aligned}
& \Lambda\left[\widehat{w}, \widehat{w}^{\prime}\right]:=-\mathrm{i}\left\langle\mathbb{J}^{-1} \widehat{W}, \widehat{w}^{\prime}\right\rangle_{r}=\mathrm{i}\left\langle\mathbb{d} \widehat{W}, \widehat{w}^{\prime}\right\rangle_{r}=\mathrm{i} \int_{\mathbb{T}}\left(\widehat{u} \widehat{v}^{\prime}-\widehat{v} \widehat{u}^{\prime}\right) \mathrm{d} x, \\
& \forall \widehat{W}=(\widehat{u}, \widehat{v}), \widehat{w}^{\prime}=\left(\widehat{u}^{\prime}, \widehat{v}^{\prime}\right) \in H_{c}^{0} .
\end{aligned}
$$

For any sufficiently smooth functionals $\mathcal{F}, \mathcal{G}: H_{c}^{0} \rightarrow \mathbb{C}$, one has

$$
\Lambda\left(X_{\mathcal{F}}, X_{\mathcal{G}}\right)=\{\mathcal{F}, \mathcal{G}\}
$$

In terms of the Fourier coefficients of $\widehat{w}$ and $\widehat{w}^{\prime}, \Lambda\left[\widehat{w}, \widehat{w}^{\prime}\right]$ can be expressed as

$$
\Lambda\left[\widehat{W}, \widehat{w}^{\prime}\right]=\mathrm{i} \sum_{k \in \mathbb{Z}}\left(\widehat{u}_{k} \widehat{V}_{-k}^{\prime}-\widehat{V}_{-k} \widehat{u}_{k}^{\prime}\right)
$$

and hence $\Lambda$ can be conveniently written as

$$
\Lambda=\mathrm{i} \sum_{k \in \mathbb{Z}} \mathrm{d} u_{k} \wedge \mathrm{d} v_{-k}
$$


where

$$
\left(\mathrm{d} u_{k} \wedge \mathrm{d} v_{-k}\right)\left[(\widehat{u}, \widehat{v}),\left(\widehat{u}^{\prime}, \widehat{v}^{\prime}\right)\right]=\widehat{u}_{k} \widehat{v}_{-k}^{\prime}-\widehat{v}_{-k} \widehat{u}_{k}^{\prime}, \quad \forall k \in \mathbb{Z}
$$

In addition, we define the one form $\lambda$ on $H_{c}^{0}$ as

$$
\lambda \equiv \lambda(w)=\mathrm{i} \sum_{k \in \mathbb{Z}} u_{k} \mathrm{~d} v_{-k}
$$

Its action on a function $\widehat{w}=(\widehat{u}, \widehat{v}) \in H_{c}^{0}$ is given by

$$
\lambda[\widehat{W}]=\mathrm{i} \int_{\mathbb{T}} u(x) \widehat{V}(x) \mathrm{d} x=\mathrm{i} \sum_{k \in \mathbb{Z}} u_{k} \widehat{V}_{-k}
$$

The exterior differential of $\lambda$, defined by $d \lambda=\mathrm{i} \sum_{k \in \mathbb{Z}} \mathrm{d} u_{k} \wedge \mathrm{d} v_{-k}$, thus satisfies $d \lambda=\Lambda$.

The Poisson bracket on the model space $h_{c}^{0}$ is determined by defining it for the coordinate functions,

$$
\left\{x_{n}, y_{m}\right\}_{M}=-\delta_{n m}, \quad\left\{y_{n}, x_{m}\right\}_{M}=\delta_{n m}, \quad\left\{x_{n}, x_{m}\right\}_{M}=0, \quad\left\{y_{n}, y_{m}\right\}_{M}=0, \quad \forall n, m \in \mathbb{Z} .
$$

By a slight abuse of terminology in connection with the definition (1.4), we also denote by $(\cdot, \cdot)_{r}$ the non-degenerate bilinear form $(\cdot, \cdot)_{r}: h_{c}^{0} \times h_{c}^{0} \rightarrow \mathbb{C}$

$$
\left(z, z^{\prime}\right)_{r}:=x \cdot x^{\prime}+y \cdot y^{\prime}, \quad \forall z=(x, y), z^{\prime}=\left(x^{\prime}, y^{\prime}\right) \in h_{c}^{0}
$$

where $x \cdot x^{\prime}:=\sum_{k \in \mathbb{Z}} x_{k} x_{k}^{\prime}$. Given two sufficiently smooth functionals $F, G: h_{c}^{0} \rightarrow \mathbb{C}$, one has

$$
\{F, G\}_{M}=-\sum_{k}\left(\partial_{x_{k}} F \partial_{y_{k}} G-\partial_{y_{k}} F \partial_{x_{k}} G\right)=(\nabla F, J \nabla G)_{r}
$$

where

$$
J:=\left(\begin{array}{cc}
0 & -\mathrm{Id} \\
\mathrm{Id} & 0
\end{array}\right): h_{c}^{0} \rightarrow h_{c^{\prime}}^{0}
$$

Id $: h_{c}^{0} \rightarrow h_{c}^{0}$ is the identity operator and

$$
\nabla F=\left(\nabla_{X} F, \nabla_{Y} F\right), \quad \nabla_{X} F=\left(\partial_{x_{k}} F\right)_{k \in \mathbb{Z}}, \quad \nabla_{Y} F=\left(\partial_{Y_{k}} F\right)_{k \in \mathbb{Z}}
$$


1484 T. Kappeler and R. Montalto

The Hamiltonian vector field $X_{F}$ of $F: h_{c}^{0} \rightarrow \mathbb{C}$, corresponding to the Poisson bracket $\{\cdot, \cdot\}_{M}$, is then given by

$$
X_{F}=J \nabla F
$$

and the symplectic form $\Lambda_{M}$, associated with it, by

$$
\Lambda_{M}\left[\widehat{z}, \widehat{z}^{\prime}\right]:=\left(J^{-1} \widehat{z}, \widehat{z}^{\prime}\right)_{r}=\widehat{y} \cdot \widehat{x}^{\prime}-\widehat{x} \cdot \widehat{y}^{\prime}, \quad \forall \widehat{z}=(\widehat{x}, \widehat{y}), \widehat{z}^{\prime}=\left(\widehat{x}^{\prime}, \widehat{y}^{\prime}\right) \in h_{c}^{0}
$$

Note that

$$
\Lambda_{M}=-\sum_{k \in \mathbb{Z}} \mathrm{d} x_{k} \wedge d y_{k}
$$

where as above, for any $k \in \mathbb{Z}$, the two form $\mathrm{d} x_{k} \wedge \mathrm{d} y_{k}$ is defined as

$$
\left(\mathrm{d} x_{k} \wedge \mathrm{d} y_{k}\right)\left[(\widehat{x}, \widehat{y}),\left(\widehat{x}^{\prime}, \widehat{y}^{\prime}\right)\right]=\widehat{x}_{k} \widehat{y}_{k}^{\prime}-\widehat{y}_{k} \widehat{x}_{k}^{\prime}
$$

Then

$$
\Lambda_{M}\left(X_{F}, X_{G}\right)=(\nabla F, J \nabla G)_{r}=\{F, G\}_{M}
$$

The one form associated with $\Lambda_{M}$ is defined as

$$
\lambda_{M} \equiv \lambda_{M}(z):=\sum_{k \in \mathbb{Z}} y_{k} \mathrm{~d} x_{k}
$$

Its action on a vector $\widehat{z}=(\widehat{x}, \widehat{y}) \in h_{c}^{0}$ is given by

$$
\lambda_{M}[\widehat{Z}]=\sum_{k \in \mathbb{Z}} y_{k} \widehat{X}_{k} .
$$

The exterior differential of $\lambda_{M}$ then satisfies $\mathrm{d} \lambda_{M}=\Lambda_{M}$.

\section{The Map $\Psi_{L}$}

In this section, we study the map $\Psi_{L}$ introduced in (1.8). In particular, we prove tame estimates and one smoothing properties for $\Psi_{L}$. First we introduce some more notations. Denote by $\Pi_{S}$ and $\Pi_{\perp}$ the standard projections

$$
\Pi_{S}:\left(\mathbb{C}^{S} \times \mathbb{C}^{S}\right) \times h_{\perp C}^{0} \rightarrow\left(\mathbb{C}^{S} \times \mathbb{C}^{S}\right) \times\{0\}, z=\left(z_{S}, z_{\perp}\right) \mapsto\left(z_{S}, 0\right)
$$




$$
\Pi_{\perp}:\left(\mathbb{C}^{S} \times \mathbb{C}^{S}\right) \times h_{\perp c}^{0} \rightarrow\{0\} \times h_{\perp c}^{0}, z=\left(z_{S}, z_{\perp}\right) \mapsto\left(0, z_{\perp}\right)
$$

The formula (1.8) for $\Psi_{L}(z)$ with $z=\left(z_{S}, z_{\perp}\right)$ then reads

$$
\Psi_{L}(z)=\Psi^{\mathrm{nls}}\left(\Pi_{S} z\right)+d_{\perp} \Psi^{\mathrm{nls}}\left(\Pi_{S} z\right)\left[z_{\perp}\right]
$$

For a quite explicit formula for $d_{\perp} \Psi^{\mathrm{nls}}\left(\Pi_{S} z\right)\left[z_{\perp}\right]$, we refer to Appendix 2. The map $\Psi_{L}$ is defined on

$$
\mathcal{V}^{\max }:=\mathcal{V}_{S}^{\max } \times h_{\perp c^{\prime}}^{s}, \quad \mathcal{V}_{S}^{\max }:=\Pi_{S} \Phi^{\mathrm{nls}}(\mathcal{W}),
$$

where $\mathcal{W} \subseteq H_{c}^{0}$ is the domain of definition of the Birkhoff map $\Phi^{\text {nls }}$ of Theorem 1.1. Note that

$$
\mathbb{R}^{S} \times \mathbb{R}^{S} \subseteq \mathcal{V}_{S}^{\max } \subseteq \mathbb{C}^{S} \times \mathbb{C}^{S}, \quad h_{r}^{0} \subset \mathcal{V}^{\max } \subset h_{c^{\prime}}^{0} \quad \Psi_{L}(0)=0
$$

Furthermore, the differential $d \Psi_{L}(z)$ of $\Psi_{L}$ at $z=\left(z_{S}, z_{\perp}\right) \in \mathcal{V}^{\max }$ applied to a vector $\widehat{z}=\left(\widehat{z}_{S}, \widehat{z}_{\perp}\right) \in h_{c}^{0}$ is given by

$$
\begin{aligned}
d \Psi_{L}(z)\left[\widehat{z}_{S}, \widehat{z}_{\perp}\right] & =d_{S} \Psi^{\mathrm{nls}}\left(\Pi_{S} z\right)\left[\widehat{z}_{S}\right]+d_{\perp} \Psi^{\mathrm{nls}}\left(\Pi_{S} z\right)\left[\widehat{z}_{\perp}\right]+d_{S}\left(d_{\perp} \Psi^{\mathrm{nls}}\left(\Pi_{S} z\right)\left[z_{\perp}\right]\right)\left[\widehat{z}_{S}\right] \\
& =\mathrm{d} \Psi^{\mathrm{nls}}\left(\Pi_{S} z\right)[\widehat{z}]+d^{2} \Psi^{\mathrm{nls}}\left(\Pi_{S} z\right)\left[\Pi_{S} \widehat{z}, \Pi_{\perp} z\right] .
\end{aligned}
$$

The latter expression is independent of $\Pi_{\perp} \widehat{z}$ and that by Theorem 1.1, $d \Psi_{L}(0)=$ $\mathrm{d} \Psi^{\mathrm{nls}}(0)=F_{\mathrm{nls}}^{-1}$. First we establish the following auxiliary results.

Lemma 3.1. (i) The map $\Psi_{L}: \mathcal{V}^{\max } \rightarrow H_{c}^{0}$ is analytic and for any $s \in \mathbb{Z}_{\geq 0}$, the restriction $\left.\Psi_{L}\right|_{h_{r}^{s}}: h_{r}^{s} \rightarrow H_{r}^{s}$ is real analytic. Furthermore, for any $z_{S} \in \mathbb{R}^{S} \times \mathbb{R}^{S}$ and any $s \in \mathbb{Z}_{\geq 0}$, $d \Psi_{L}\left(z_{S}, 0\right): h_{c}^{s} \rightarrow H_{c}^{s}$ is a linear isomorphism.

(ii) For any compact subset $\mathcal{K} \subseteq \mathbb{R}^{S} \times \mathbb{R}^{S}$, there exists a ball $\mathcal{V}_{\perp}$ in $h_{\perp r}^{0}$, centred at 0 , so that the restriction $\Psi_{L}: \mathcal{K} \times \mathcal{V}_{\perp} \rightarrow H_{r}^{0}$ is one to one. Furthermore, after shrinking the radius of the ball $\mathcal{V}_{\perp}$, if necessary, the map $\Psi_{L}: \mathcal{K} \times \mathcal{V}_{\perp} \rightarrow H_{r}^{0}$ is a local diffeomorphism.

Proof. (i) The claimed analyticity follows from the definition of $\Psi_{L}$ and the corresponding properties of $\Psi^{\text {nls }}$, stated in Theorem 1.1. Concerning the statement on the differential $d \Psi_{L}\left(z_{S}, 0\right)$, note that by (3.5), $d \Psi_{L}\left(z_{S}, 0\right)=\mathrm{d} \Psi^{\text {nls }}\left(z_{S}, 0\right)$ and hence by Theorem $1.1, d \Psi_{L}\left(z_{S}, 0\right): h_{c}^{s} \rightarrow H_{c}^{s}$ is a linear isomorphism for any $s \in \mathbb{Z}_{\geq 0}$. 
(ii) Let $\mathcal{K} \subseteq \mathbb{R}^{S} \times \mathbb{R}^{S}$ be a given compact subset. Assume that there exists no ball $\mathcal{V}_{\perp}$ in $h_{\perp r}^{0}$, centred at 0 , so that $\left.\Psi_{L}\right|_{\mathcal{K} \times \mathcal{V}_{\perp}}$ is one to one. Then there exist two sequences $z^{(j)}=\left(z_{S}^{(j)}, z_{\perp}^{(j)}\right)$, $j \geq 1$, and $\tilde{z}^{(j)}=\left(\tilde{z}_{S}^{(j)}, \tilde{z}_{\perp}^{(j)}\right), j \geq 1$, in $\mathcal{K} \times h_{\perp r}^{0}$ such that for any $j \geq 1$

$$
z^{(j)} \neq \tilde{z}^{(j)}, \quad \Psi_{L}\left(z^{(j)}\right)=\Psi_{L}\left(\tilde{z}^{(j)}\right), \quad \lim _{j \rightarrow \infty} z_{\perp}^{(j)}=\lim _{j \rightarrow \infty} \tilde{z}_{\perp}^{(j)}=0 .
$$

Since by assumption $\mathcal{K}$ is compact, there exist subsequences of $\left(z^{(j)}\right)_{j \geq 1},\left(\tilde{z}^{(j)}\right)_{j \geq 1}$, denoted for simplicity in the same way, such that $\left(z_{S}^{(j)}\right)_{j \geq 1},\left(\tilde{z}_{S}^{(j)}\right)_{j \geq 1}$ converge. Denote their limits by $z_{S}^{(\infty)}$ and $\tilde{z}_{S}^{(\infty)}$, respectively. Then

$$
\lim _{j \rightarrow \infty} z^{(j)}=\left(z_{S}^{(\infty)}, 0\right), \quad \lim _{j \rightarrow \infty} \tilde{z}^{(j)}=\left(\tilde{z}_{S}^{(\infty)}, 0\right)
$$

are elements in $\mathcal{K} \times\{0\}$. By the continuity of $\Psi_{L}$, one has $\Psi_{L}\left(z_{S}^{(\infty)}, 0\right)=\Psi_{L}\left(\tilde{z}_{S}^{(\infty)}, 0\right)$ and since $\Psi_{L}$ and $\Psi^{\text {nls }}$ coincide on $\mathcal{V}_{S}^{\max } \times\{0\}$ it then follows from Theorem 1.1 that $z_{S}^{(\infty)}=\tilde{z}_{S}^{(\infty)}$. By item (i) and the local inversion theorem one then concludes that in contradiction to our assumption, $z^{(j)}=\tilde{z}^{(j)}$ for $j$ sufficiently large. This proves the first part of item (ii). Since according to item (i), for any given $z_{S} \in \mathcal{K}, d \Psi_{L}\left(z_{S}, 0\right): h_{c}^{0} \rightarrow H_{c}^{0}$ is a linear isomorphism, $d \Psi_{L}(z)$ is such an operator for $z$ in a whole neighbourhood of $\left(z_{S}, 0\right)$. Using that $\mathcal{K}$ is compact it then follows that after shrinking the radius of the ball $\mathcal{V}_{\perp}$, if necessary, $\Psi_{L}: \mathcal{K} \times \mathcal{V}_{\perp} \rightarrow H_{r}^{0}$ is a local diffeomorphism.

Proposition 3.1. For any compact subset $\mathcal{K} \subseteq \mathbb{R}^{S} \times \mathbb{R}^{S}$ there exists an open complex neighbourhood $\mathcal{V}$ of $\mathcal{K} \times\{0\}$ in $h_{c}^{0}$ of the form $\mathcal{V}_{S} \times \mathcal{V}_{\perp}$ where $\overline{\mathcal{V}}_{S}$ is compact with $\overline{\mathcal{V}}_{S} \subseteq \mathcal{V}_{S}^{\text {max }}$ and $\mathcal{V}_{\perp} \subset h_{\perp c}^{0}$ is an open ball, centred at 0 , so that the restriction of $\Psi_{L}$ to $\mathcal{V}$ has the following properties:

$(L 1) \Psi_{L}$ is analytic on $\mathcal{V}$ and

$$
\Psi_{L}\left|\mathcal{V}_{S} \times\{0\}=\Psi^{\mathrm{nls}}\right|_{\mathcal{V}_{S} \times\{0\}}, \quad \mathrm{d} \Psi_{L}\left(z_{S}, 0\right)=\mathrm{d} \Psi^{\mathrm{nls}}\left(z_{S}, 0\right), \quad \forall z_{S} \in \mathcal{V}_{S}
$$

Furthermore, $\Psi_{L}: \mathcal{V} \cap h_{r}^{0} \rightarrow H_{r}^{0}$ is a real analytic diffeomorphism onto its image.

(L2) The map $B_{L}:=\Psi_{L}-F_{\text {nls }}^{-1}: \mathcal{V} \rightarrow H_{c}^{0}$ is analytic and one smoothing. More precisely, the analytic map $B_{L}$ is given by

$$
B_{L}(z)=B^{\mathrm{nls}}\left(\Pi_{S} z\right)+d_{\perp} B^{\mathrm{nls}}\left(\Pi_{S} z\right)\left[z_{\perp}\right]
$$

with $B^{\text {nls }}$ being the map introduced in Theorem 1.1, and for any $s \in \mathbb{Z}_{\geq 1}, B_{L}: \mathcal{V} \cap h_{r}^{s} \rightarrow H_{r}^{s+1}$ is real analytic. Furthermore

$$
d_{\perp} B_{L}(z)=d_{\perp} B^{\mathrm{nls}}\left(\Pi_{S} z\right), \quad d_{\perp}^{2} B_{L}(z)=0, \quad \forall z \in \mathcal{V}
$$


and for any $z \in \mathcal{V} \cap h_{r}^{0}, \alpha, \beta \in \mathbb{Z}_{\geq 0}^{S}$,

$$
\left\|\partial_{S}^{\alpha, \beta} B_{L}(z)\right\|_{0} \lesssim_{\alpha, \beta} \quad 1, \quad\left\|\partial_{S}^{\alpha, \beta} d_{\perp} B_{L}(z)\left[\widehat{z}_{\perp}\right]\right\|_{0} \lesssim_{\alpha, \beta}\left\|\widehat{z}_{\perp}\right\|_{0}, \quad \forall \widehat{z}_{\perp} \in h_{\perp c}^{0}
$$

and for any $s \in \mathbb{Z}_{\geq 1}, z \in \mathcal{V} \cap h_{r}^{s}$

$$
\left\|\partial_{S}^{\alpha, \beta} B_{L}(z)\right\|_{s+1} \lesssim_{s, \alpha, \beta} 1+\left\|z_{\perp}\right\|_{s,} \quad\left\|\partial_{S}^{\alpha, \beta} d_{\perp} B_{L}(z)\left[\widehat{z}_{\perp}\right]\right\|_{s+1} \lesssim_{s, \alpha, \beta}\left\|\widehat{z}_{\perp}\right\|_{s,} \quad \forall \widehat{z}_{\perp} \in h_{\perp c}^{s} .
$$

(L3) For any $s \in \mathbb{Z}_{\geq 1}$, the restriction $\left.\Psi_{L}\right|_{\mathcal{V} \cap h_{r}^{s}}$ is a map $\mathcal{V} \cap h_{r}^{s} \rightarrow H_{r}^{s}$ which is a real analytic diffeomorphism onto its image.

(L4) The map $A_{L}:=\Psi_{L}^{-1}-F_{\mathrm{nls}}: \Psi_{L}(\mathcal{V}) \rightarrow h_{c}^{0}$ is analytic and one smoothing, meaning that for any $s \in \mathbb{Z}_{\geq 1}, A_{L}: \Psi_{L}(\mathcal{V}) \cap H_{r}^{s} \rightarrow h_{r}^{s+1}$ is real analytic.

Remark 3.1. For convenience, in the sequel, we always choose $\mathcal{V}_{\perp}$ to be a ball of radius smaller than one.

Proof. Choose $\mathcal{V}_{S}$ to be an open bounded neighbourhood of $\mathcal{K}$ in $\mathbb{C}^{S} \times \mathbb{C}^{S}$ so that $\overline{\mathcal{V}}_{S} \subseteq \mathcal{V}_{S}^{\max }$ and let $\mathcal{V}_{\perp}$ be an open ball in $h_{\perp C^{\prime}}^{0}$, centred at 0, so that item (ii) of Lemma 3.1 applies to $\mathcal{V}:=\mathcal{V}_{S} \times \mathcal{V}_{\perp}$, implying that $\Psi_{L}: \mathcal{V} \cap h_{r}^{0} \rightarrow H_{r}^{0}$ is one to one and a local diffeomorphism. The identities (3.6) hold by the definition of $\Psi_{L}$ and the analyticity of $\Psi_{L}$, stated in ( $\left.L 1\right)$, follows by Lemma 3.1(i). One then concludes that

$$
\Psi_{L}: \mathcal{V} \cap h_{r}^{0} \rightarrow H_{r}^{0}
$$

is a real analytic diffeomorphism on to its image. (L2) follows from the definition of $\Psi_{L}$, Theorem 1.1, the compactness of $\overline{\mathcal{V}}_{S}$, and standard estimates in Sobolev spaces. Concerning ( $L 3$ ), first note that by Theorem 1.1, for any $s \in \mathbb{Z}_{\geq 1}$, the restriction $\left.\Psi_{L}\right|_{\mathcal{V} \cap h_{r}^{s}}$ is a map with values in $H_{r}^{s}$ and as such real analytic. By item (L1), $\left.\Psi_{L}\right|_{\mathcal{V} \cap h_{r}^{s}}$ is one to one and so is its differential $d \Psi_{L}(z): h_{c}^{s} \rightarrow H_{c}^{s}$ at any point $z \in \mathcal{V} \cap h_{r}^{s}$. Since by (L2) the map $B_{L}$ is one smoothing, $d \Psi_{L}(z): h_{c}^{s} \rightarrow H_{c}^{s}$ is Fredholm and hence a linear isomorphism, implying that $\Psi_{L}: \mathcal{V} \cap h_{r}^{s} \rightarrow H_{r}^{s}$ is a real analytic diffeomorphism on to its image. Finally, item (L4) follows from (L3) and Theorem 1.1.

Whereas the tame estimates (3.9) for $B_{L}$ are an immediate consequence of the definition of $\Psi_{L}$, Theorem 1.1 and the compactness of $\overline{\mathcal{V}}_{S}$, this is not so for $A_{L}$. Actually, for the applications in perturbation theory considered in subsequent work, we only need to derive tame estimates for

$$
\mathcal{A}_{L}: \mathcal{V} \cap h_{r}^{0} \rightarrow \mathcal{L}\left(H_{c}^{0}, h_{c}^{0}\right), \quad z \mapsto \mathcal{A}_{L}(z):=\mathrm{d} A_{L}\left(\Psi_{L}(z)\right)=\mathrm{d} \Psi_{L}(z)^{-1}-F_{\mathrm{nls}}
$$


with $\mathcal{V}$ denoting the neighbourhood of $\mathcal{K} \times\{0\}$ of Proposition 3.1. By formula (3.5), for any $z \in \mathcal{V} \cap h_{r}^{0}$, the operator $d \Psi_{L}(z) \in \mathcal{L}\left(h_{c}^{0}, H_{c}^{0}\right)$ can be written as

$$
\mathrm{d} \Psi_{L}(z)=\mathcal{T}(z)+\mathcal{R}(z), \quad \mathcal{T}(z):=\mathrm{d} \Psi^{\mathrm{nls}}\left(\Pi_{S} z\right)
$$

with $\mathcal{R}(z) \in \mathcal{L}\left(h_{c}^{0}, H_{c}^{0}\right)$ given by

$$
\mathcal{R}(z): h_{c}^{0} \rightarrow H_{c}^{0}, \quad \widehat{z} \mapsto \mathcal{R}(z)[\widehat{z}]:=\mathrm{d}^{2} \Psi^{\mathrm{nls}}\left(\Pi_{S} z\right)\left[\Pi_{S} \widehat{z}, \Pi_{\perp} z\right]=\mathrm{d}^{2} B^{\mathrm{nls}}\left(\Pi_{S} z\right)\left[\Pi_{S} \widehat{z}, \Pi_{\perp} z\right]
$$

Since by Theorem 1.1, respectively Proposition 3.1, the operators $\mathcal{T}(z), \mathrm{d} \Psi_{L}(z): h_{c}^{0} \rightarrow H_{c}^{0}$ are invertible, so is $\mathcal{T}(z)^{-1} \mathrm{~d} \Psi_{L}(z)=\mathrm{Id}+\mathcal{T}(z)^{-1} \mathcal{R}(z)$, implying that

$$
\mathrm{d} \Psi_{L}(z)^{-1}=\left(\operatorname{Id}+\mathcal{T}(z)^{-1} \mathcal{R}(z)\right)^{-1} \mathcal{T}(z)^{-1},=\mathcal{T}(z)^{-1}-\mathcal{T}(z)^{-1} \mathcal{R}(z) \mathcal{S}(z)
$$

where

$$
\mathcal{S}(z):=\left(\operatorname{Id}+\mathcal{T}(z)^{-1} \mathcal{R}(z)\right)^{-1} \mathcal{T}(z)^{-1} \in \mathcal{L}\left(H_{c}^{0}, h_{c}^{0}\right)
$$

Furthermore, by Theorem 1.1

$$
\mathcal{T}(z)^{-1}=\left(\mathrm{d} \Psi^{\mathrm{nls}}\left(\Pi_{S} z\right)\right)^{-1}=\mathrm{d} \Phi^{\mathrm{nls}}\left(\Pi_{S} z\right)=F_{\mathrm{nls}}+\mathrm{d} A^{\mathrm{nls}}\left(\Psi^{\mathrm{nls}}\left(\Pi_{S} z\right)\right)
$$

Altogether, it follows that for any $z \in \mathcal{V} \cap h_{r}^{0}$, the operator $\mathcal{A}_{L}(z)=\mathrm{d} \Psi_{L}(z)^{-1}-F_{\mathrm{nls}}: h_{c}^{0} \rightarrow H_{c}^{0}$ can be written as

$$
\mathcal{A}_{L}(z)=\mathrm{d} A^{\mathrm{nls}}\left(\Psi^{\mathrm{nls}}\left(\Pi_{S} z\right)\right)-\mathcal{T}(z)^{-1} \mathcal{R}(z) \mathcal{S}(z)
$$

Finally we note that by ( $L 4)$ of Proposition 3.1, $\mathcal{A}_{L}=\mathrm{d} A_{L} \circ \Psi_{L}$ is one smoothing. More precisely, for any $s \in \mathbb{Z}_{\geq 1}$, the restriction of $\mathcal{A}_{L}$ to $\mathcal{V} \cap h_{r}^{s}$ is a real analytic map,

$$
\mathcal{A}_{L}: \mathcal{V} \cap h_{r}^{s} \rightarrow \mathcal{L}\left(H_{c}^{s}, h_{c}^{s+1}\right), \quad z \mapsto \mathcal{A}_{L}(z)
$$

Proposition 3.2 (Tame estimates for $\mathcal{A}_{L}$ ). After shrinking, if necessary, the radius of the ball $\mathcal{V}_{\perp}$ in $\mathcal{V}=\mathcal{V}_{S} \times \mathcal{V}_{\perp}$ of Proposition 3.1, the map $\mathcal{A}_{L}$ satisfies for any $z \in \mathcal{V} \cap h_{r}^{0}$, $\widehat{W} \in h_{c^{\prime}}^{0}$

$$
\left\|\mathcal{A}_{L}(z)[\widehat{W}]\right\|_{0} \lesssim\|\widehat{W}\|_{0}
$$


and for any $k \in \mathbb{Z}_{\geq 1}, \widehat{z}_{1}, \ldots, \widehat{z}_{k} \in h_{c^{\prime}}^{0}$

$$
\left\|d^{k}\left(\mathcal{A}_{L}(z)[\widehat{W}]\right)\left[\widehat{z}_{1}, \ldots, \widehat{Z}_{k}\right]\right\|_{0} \lesssim_{k}\|\widehat{W}\|_{0} \prod_{j=1}^{k}\left\|\widehat{z}_{j}\right\|_{0}
$$

Furthermore, for any $s \in \mathbb{Z}_{\geq 1}, z \in \mathcal{V} \cap h_{r}^{s}, \widehat{w} \in H_{c}^{s}$,

$$
\left\|\mathcal{A}_{L}(z)[\widehat{W}]\right\|_{s+1} \lesssim_{s}\left\|z_{\perp}\right\|_{s}\|\widehat{W}\|_{0}+\|\widehat{W}\|_{s}
$$

and for any $k \geq 1, \widehat{z}_{1}, \ldots, \widehat{z}_{k} \in h_{c^{\prime}}^{s}$

$$
\left\|d^{k}\left(\mathcal{A}_{L}(z)[\widehat{w}]\right)\left[\widehat{z}_{1}, \ldots, \widehat{z}_{k}\right]\right\|_{s+1} \lesssim_{s, k} \quad\left(\left\|z_{\perp}\right\|_{s}\|\widehat{w}\|_{0}+\|\widehat{w}\|_{s}\right) \prod_{j=1}^{k}\left\|\widehat{z}_{j}\right\|_{0}+\|\widehat{w}\|_{0} \sum_{j=1}^{k}\left\|\widehat{z}_{j}\right\|_{s} \prod_{i \neq j}\left\|\widehat{z}_{i}\right\|_{0} .
$$

Proof. First we prove estimate (3.16). The starting point is formula (3.15) for $\mathcal{A}_{L}(z)$. The two terms $d A^{\text {nls }}\left(\Psi^{\text {nls }}\left(\Pi_{S} z\right)\right)$ and $\mathcal{T}(z)^{-1} \mathcal{R}(z) \mathcal{S}(z)$ are estimated separately. By Theorem $1.1,\left\{\Psi^{\text {nls }}\left(\Pi_{S} z\right) \mid z \in \mathcal{V} \cap h_{r}^{0}\right\}$ is a relatively compact subset of $H_{r}^{s}$ for any $s \in \mathbb{Z}_{\geq 0}$, and $A^{\text {nls }}$, $B^{\text {nls }}$ are one smoothing maps. It implies that for any $s \in \mathbb{Z}_{\geq 1}$,

$$
\left\|\mathrm{d} A^{\mathrm{nls}}\left(\Psi^{\mathrm{nls}}\left(\Pi_{S} z\right)\right)[\widehat{W}]\right\|_{s+1} \lesssim s\|\widehat{W}\|_{s}, \quad \forall z \in \mathcal{V} \cap h_{r}^{0}, \quad \forall \widehat{W} \in H_{c}^{s}
$$

Since $\left\|\Pi_{S} \widehat{z}\right\|_{s} \lesssim_{s}\left\|\Pi_{S} \widehat{z}\right\|_{0}$ for any $z \in h_{c}^{0}$, the linear operator $\mathcal{R}(z)$, defined in (3.12), satisfies

$$
\|\mathcal{R}(z)[\widehat{z}]\|_{s+1} \lesssim_{s}\left\|z_{\perp}\right\|_{s}\left\|\Pi_{S} \widehat{z}\right\|_{s} \lesssim_{s}\left\|z_{\perp}\right\|_{s}\left\|\Pi_{S} \widehat{z}\right\|_{0} \quad \forall z \in \mathcal{V} \cap h_{r}^{0} \quad \forall \widehat{z} \in h_{c}^{0}
$$

Furthermore, also by Theorem 1.1, one has for any $s \in \mathbb{Z}_{\geq 0}$,

$$
\left\|\mathcal{T}(z)^{-1}[\widehat{W}]\right\|_{s} \lesssim s\|\widehat{W}\|_{s}, \quad \forall z \in \mathcal{V} \cap h_{r}^{0}, \quad \forall \widehat{W} \in H_{c}^{s}
$$


Combining (3.18-3.20), formula (3.15) leads to the estimate

$$
\begin{aligned}
\left\|\mathcal{A}_{L}(z)[\widehat{w}]\right\|_{s+1} & \leq\left\|\mathrm{d} A^{\mathrm{nls}}\left(\Psi^{\mathrm{nls}}\left(\Pi_{S} z\right)\right)[\widehat{w}]\right\|_{s+1}+\left\|\mathcal{T}(z)^{-1} \mathcal{R}(z) \mathcal{S}(z)[\widehat{w}]\right\|_{s+1} \\
& \lesssim s \widehat{w}\left\|_{s}+\right\| z_{\perp}\left\|_{s}\right\| \Pi_{S} \mathcal{S}(z)[\widehat{w}] \|_{0}
\end{aligned}
$$

It remains to estimate $\|\mathcal{S}(z)[\widehat{W}]\|_{0}$. Recall that by (3.14), $\mathcal{S}(z)=\left(\operatorname{Id}+\mathcal{T}(z)^{-1} \mathcal{R}(z)\right)^{-1} \mathcal{T}(z)^{-1}$. By Theorem 1.1 there exists $C_{0}>0$ so that

$$
\left\|\mathcal{T}(z)^{-1} \mathcal{R}(z)[\widehat{z}]\right\|_{0} \leq C_{0}\left\|z_{\perp}\right\|_{0}\left\|\Pi_{S} \widehat{z}\right\|_{0} \quad \forall z \in \mathcal{V} \cap h_{r}^{0}, \quad \forall \widehat{z} \in h_{c}^{0} .
$$

Shrinking the radius of the ball $\mathcal{V}_{\perp}$ in $h_{\perp C^{\prime}}^{0}$, if necessary, so that $C_{0}\left\|z_{\perp}\right\|_{0} \leq 1 / 2$ for any $z_{\perp} \in \mathcal{V}_{\perp}$, the Neumann series of the operator $\left(\operatorname{Id}+\mathcal{T}(z)^{-1} \mathcal{R}(z)\right)^{-1}$ absolutely converges in $\mathcal{L}\left(h_{c}^{0}, h_{c}^{0}\right)$ and the operator norm of $\left(\operatorname{Id}+\mathcal{T}(z)^{-1} \mathcal{R}(z)\right)^{-1}$ in $\mathcal{L}\left(h_{c}^{0}, h_{c}^{0}\right)$ is bounded by 2. Hence

$$
\|\mathcal{S}(z)[\widehat{W}]\|_{0} \lesssim_{s}\|\widehat{W}\|_{0}, \quad \forall z \in \mathcal{V} \cap h_{r}^{0}, \quad \forall \widehat{W} \in H_{c}^{0}
$$

implying together with (3.21) the claimed estimate (3.16).

Finally let us prove the estimate (3.17) for the derivatives of $\mathcal{A}_{L}(z)$. By formula (3.15) for any $k, s \in \mathbb{Z}_{\geq 1}, z \in \mathcal{V} \cap h_{r}^{s}, \widehat{w} \in H_{c}^{s}$, and $\widehat{z}_{1}, \ldots, \widehat{z}_{k} \in h_{c}^{s}$,

$$
\begin{aligned}
\left\|\mathrm{d}^{k}\left(\mathcal{A}_{L}(z)[\widehat{w}]\right)\left[\widehat{z}_{1}, \ldots, \widehat{z}_{k}\right]\right\|_{s+1} \leq & \left\|\mathrm{d}^{k}\left(\mathrm{~d} A^{\mathrm{nls}}\left(\Psi^{\mathrm{nls}}\left(\Pi_{S} z\right)\right)[\widehat{w}]\right)\left[\widehat{z}_{1}, \ldots, \widehat{z}_{k}\right]\right\|_{s+1} \\
& +\left\|\mathrm{d}^{k}\left(\mathcal{T}(z)^{-1} \mathcal{R}(z) \mathcal{S}(z)[\widehat{w}]\right)\left[\widehat{z}_{1}, \ldots, \widehat{z}_{k}\right]\right\|_{s+1}
\end{aligned}
$$

By Theorem 1.1, one concludes that

$$
\left\|\mathrm{d}^{k}\left(\mathrm{~d} A^{\mathrm{nls}}\left(\Psi_{L}\left(\Pi_{S} z\right)\right)[\widehat{w}]\right)\left[\widehat{z}_{1}, \ldots, \widehat{z}_{k}\right]\right\|_{s+1} \lesssim s, k\|\widehat{W}\|_{s} \prod_{j=1}^{k}\left\|\widehat{z}_{j}\right\|_{0}
$$

Furthermore

$$
\begin{gathered}
\left\|\mathrm{d}^{k}\left(\mathcal{T}(z)^{-1}[\widehat{W}]\right)\left[\widehat{z}_{1}, \ldots, \widehat{z}_{k}\right]\right\|_{s} \lesssim_{s, k}\|\widehat{W}\|_{s} \prod_{j=1}^{k}\left\|\widehat{Z}_{j}\right\|_{0}, \\
\left\|\mathrm{~d}^{k}(\mathcal{R}(\boldsymbol{z})[\widehat{Z}])\left[\widehat{Z}_{1}, \ldots, \widehat{Z}_{k}\right]\right\|_{s+1} \lesssim_{s, k}\left\|z_{\perp}\right\|_{s}\|\widehat{Z}\|_{0} \prod_{j=1}^{k}\left\|\widehat{z}_{j}\right\|_{0}+\|\widehat{Z}\|_{0} \sum_{j=1}^{k}\left\|\widehat{z}_{j}\right\|_{s} \prod_{i \neq j}\left\|\widehat{Z}_{j}\right\|_{0},
\end{gathered}
$$


and

$$
\left\|\mathrm{d}^{k}(\mathcal{S}(z)[\widehat{w}])\left[\widehat{z}_{1}, \ldots, \widehat{z}_{k}\right]\right\|_{0} \lesssim s\|\widehat{W}\|_{0} \prod_{j=1}^{k}\left\|\widehat{z}_{j}\right\|_{0} .
$$

Combining the estimates (3.26-3.28) and using the product rule implies that

$$
\begin{aligned}
& \left\|\mathrm{d}^{k}\left(\mathcal{T}(z)^{-1} \mathcal{R}(z) \mathcal{S}(z)[\widehat{W}]\right)\left[\widehat{Z}_{1}, \ldots, \widehat{Z}_{k}\right]\right\|_{s+1} \lesssim_{s, k} \\
& \left(\left\|\boldsymbol{Z}_{\perp}\right\|_{s}\|\widehat{W}\|_{0}+\|\widehat{W}\|_{s}\right) \prod_{j=1}^{k}\left\|\widehat{Z}_{j}\right\|_{0}+\|\widehat{W}\|_{0} \sum_{j=1}^{k}\left\|\widehat{Z}_{j}\right\|_{s} \prod_{i \neq j}\left\|\widehat{Z}_{i}\right\|_{0} .
\end{aligned}
$$

The three estimates (3.24), (3.25), (3.29) together yield (3.17).

In the remaining part of this section we describe the pullback $\Psi_{L}^{*} \Lambda$ by $\Psi_{L}$ of the standard symplectic form $\Lambda$ on $H_{r}^{0}$, introduced in (2.3). It turns out that $\Psi_{L}^{*} \Lambda$ is not the symplectic form $\Lambda_{M}$ of (2.7), making it necessary to construct the symplectic corrector $\Psi_{C}$ (see Section 4).

Given a bounded linear operator $\mathcal{P}: h_{c}^{0} \rightarrow h_{c}^{0}$, its transpose $\mathcal{P}^{t}: h_{c}^{0} \rightarrow h_{c}^{0}$ is defined to be the operator determined by

$$
\left(\mathcal{P}[\widehat{z}], \widehat{z}^{\prime}\right)_{r}=\left(\widehat{z}, \mathcal{P}^{t}\left[\widehat{z}^{\prime}\right]\right)_{r}, \quad \forall \widehat{z}, \widehat{z}^{\prime} \in h_{c}^{0}
$$

where the bilinear form $(\cdot, \cdot)_{r}$ on $h_{c}^{0}$ is defined in (2.4). Similarly, for a bounded linear operator $\mathcal{Q}: h_{c}^{0} \rightarrow H_{c}^{0}$, we denote its transpose by $\mathcal{Q}^{t}: H_{c}^{0} \rightarrow h_{c}^{0}$, determined by

$$
\langle\mathcal{Q}[\widehat{z}], \widehat{W}\rangle_{r}=\left(\widehat{z}, \mathcal{Q}^{t}[\widehat{W}]\right)_{r}, \quad \forall \widehat{Z} \in h_{c}^{0}, \widehat{W} \in H_{c}^{0}
$$

where the bilinear form $\langle\cdot, \cdot\rangle_{r}$ on $H_{c}^{0}$ is the one introduced in (2.2). We now compute the pullback $\Psi_{L}^{*} \Lambda(z)$ at $z=\left(z_{S}, z_{\perp}\right) \in h_{c}^{0}$ applied to $\widehat{z}=\left(\widehat{z}_{S}, \widehat{z}_{\perp}\right), \widehat{z}^{\prime}=\left(\widehat{z}_{S}^{\prime}, \widehat{z}_{\perp}^{\prime}\right)$. By the definition of the pullback and the one of $\Lambda$ in (2.3) we have

$$
\Psi_{L}^{*} \Lambda(z)\left[\widehat{z}, \widehat{z}^{\prime}\right]=\Lambda\left(\Psi_{L}(z)\right)\left[\mathrm{d} \Psi_{L}(z)[\widehat{z}], \mathrm{d} \Psi_{L}(z)\left[\widehat{z}^{\prime}\right]\right]=\mathrm{i}\left\langle J \mathrm{~d} \Psi_{L}(z)[\widehat{z}], \mathrm{d} \Psi_{L}(z)\left[\widehat{z}^{\prime}\right]\right\rangle_{r}
$$

By formula (3.5) for $d \Psi_{L}(z)$,

$$
\mathrm{d} \Psi_{L}(z)[\widehat{z}]=\mathrm{d} \Psi^{\mathrm{nls}}\left(\Pi_{S} z\right)[\widehat{z}]+d_{S}\left(d_{\perp} \Psi^{\mathrm{nls}}\left(\Pi_{S} z\right)\left[z_{\perp}\right]\right)\left[\widehat{z}_{S}\right],
$$

one gets

$$
\Psi_{L}^{*} \Lambda(z)\left[\widehat{z}, \widehat{z}^{\prime}\right]=(\mathrm{I})+(\mathrm{II})+(\mathrm{III})+(\mathrm{IV}),
$$


where

$$
\begin{gathered}
(\mathrm{I}):=\mathrm{i}\left\langle d \mathrm{~d} \Psi^{\mathrm{nls}}\left(\Pi_{S} z\right)[\widehat{z}], \mathrm{d} \Psi^{\mathrm{nls}}\left(\Pi_{S} z\right)\left[\widehat{z}^{\prime}\right]\right\rangle_{r}=\left(\left(\Psi^{\mathrm{nls}}\right)^{*} \Lambda\right)\left(\Pi_{S} z\right)\left[\widehat{z}, \widehat{z}^{\prime}\right], \\
(\mathrm{II}):=\mathrm{i}\left\langle\int \mathrm{d} \Psi^{\mathrm{nls}}\left(\Pi_{S} z\right)[\widehat{z}], d_{S}\left(d_{\perp} \Psi^{\mathrm{nls}}\left(\Pi_{S} z\right)\left[z_{\perp}\right]\right)\left[\widehat{z}_{S}^{\prime}\right]\right\rangle_{r} .
\end{gathered}
$$

Writing $d \Psi^{\mathrm{nls}}\left(\Pi_{S} z\right)[\widehat{z}]$ as $d_{S} \Psi^{\mathrm{nls}}\left(\Pi_{S} z\right)\left[\widehat{z}_{S}\right]+d_{\perp} \Psi^{\mathrm{nls}}\left(\Pi_{S} z\right)\left[\widehat{z}_{\perp}\right]$ one gets

$$
\begin{aligned}
(\mathrm{II}) & =\mathrm{i}\left\langle\int d_{S} \Psi^{\mathrm{nls}}\left(\Pi_{S} z\right)\left[\widehat{z}_{S}\right], d_{S}\left(d_{\perp} \Psi^{\mathrm{nls}}\left(\Pi_{S} z\right)\left[z_{\perp}\right]\right)\left[\widehat{z}_{S}^{\prime}\right]\right\rangle_{r} \\
& +\mathrm{i}\left\langle d d_{\perp} \Psi^{\mathrm{nls}}\left(\Pi_{S} z\right)\left[\widehat{z}_{\perp}\right], d_{S}\left(d_{\perp} \Psi^{\mathrm{nls}}\left(\Pi_{S} z\right)\left[z_{\perp}\right]\right)\left[\widehat{z}_{S}^{\prime}\right]\right\rangle_{r} .
\end{aligned}
$$

Similarly one has

$$
\begin{aligned}
(\mathrm{III}):=\mathrm{i} & \left.\left\langle\mathbb{J} d_{S}\left(d_{\perp} \Psi^{\mathrm{nls}}\left(\Pi_{S} z\right)\left[z_{\perp}\right]\right)\left[\widehat{z}_{S}\right], \mathrm{d} \Psi^{\mathrm{nls}}\left(\Pi_{S} z\right)\left[\widehat{z}^{\prime}\right]\right)\right\rangle_{r} \\
& =\mathrm{i}\left\langle\mathbb{J} d_{S}\left(d_{\perp} \Psi^{\mathrm{nls}}\left(\Pi_{S} z\right)\left[z_{\perp}\right]\right)\left[\widehat{z}_{S}\right], d_{S} \Psi^{\mathrm{nls}}\left(\Pi_{S} z\right)\left[\widehat{z}_{S}^{\prime}\right]\right\rangle_{r} \\
& +\mathrm{i}\left\langle J d_{S}\left(d_{\perp} \Psi^{\mathrm{nls}}\left(\Pi_{S} z\right)\left[z_{\perp}\right]\right)\left[\widehat{z}_{S}\right], d_{\perp} \Psi^{\mathrm{nls}}\left(\Pi_{S} z\right)\left[\widehat{z}_{\perp}^{\prime}\right]\right\rangle_{r}
\end{aligned}
$$

and finally

$$
(\mathrm{IV}):=\mathrm{i}\left\langle\mathbb{J} d_{S}\left(d_{\perp} \Psi^{\mathrm{nls}}\left(\Pi_{S} z\right)\left[z_{\perp}\right]\right)\left[\widehat{z}_{S}\right], d_{S}\left(d_{\perp} \Psi^{\mathrm{nls}}\left(\Pi_{S} z\right)\left[z_{\perp}\right]\right)\left[\widehat{z}_{S}^{\prime}\right]\right\rangle_{r}
$$

Since by Theorem $1.1, \Psi^{\text {nls }}$ is symplectic, one has $\left(\Psi^{\text {nls }}\right)^{*} \Lambda=\Lambda_{M}$. Hence for any $z \in \mathcal{V}$, $\Psi_{L}^{*} \Lambda(z)$ can be written as

$$
\Psi_{L}^{*} \Lambda(z)=\Lambda_{M}+\Lambda_{L}(z), \quad \Lambda_{L}(z)\left[\widehat{z}, \widehat{z}^{\prime}\right]:=\left(L(z)[\widehat{z}], \widehat{z}^{\prime}\right)_{r^{\prime}}
$$

where $L(z): \mathbb{C}^{S} \times \mathbb{C}^{S} \times h_{\perp c}^{0} \rightarrow \mathbb{C}^{S} \times \mathbb{C}^{S} \times h_{\perp c}^{0}$ is the linear operator of the form

$$
L(z)=\left(\begin{array}{cc}
L_{S}^{S}(z) & L_{S}^{\perp}(z) \\
L_{\perp}^{S}(z) & 0
\end{array}\right)
$$

By the computations above, $L_{S}^{S}(z): \mathbb{C}^{S} \times \mathbb{C}^{S} \rightarrow \mathbb{C}^{S} \times \mathbb{C}^{S}, L_{S}^{\perp}(z): h_{\perp c}^{0} \rightarrow \mathbb{C}^{S} \times \mathbb{C}^{S}$, and $L_{\perp}^{S}(z): \mathbb{C}^{S} \times \mathbb{C}^{S} \rightarrow h_{\perp C}^{0}$ are the linear operators defined by $\left(z \in \mathcal{V} \cap h_{r}^{0}, \widehat{z}_{S} \in \mathbb{C}^{S} \times \mathbb{C}^{S}, \widehat{z}_{\perp} \in h_{\perp c}^{0}\right)$

$$
\begin{aligned}
& L_{S}^{S}(z)\left[\widehat{z}_{S}\right]:=\mathrm{i}\left(\begin{array}{l}
\left(\left\langle J d_{S} \Psi^{\mathrm{nls}}\left(\Pi_{S} z\right)\left[\widehat{z}_{S}\right], \partial_{x_{j}} d_{\perp} \Psi^{\mathrm{nls}}\left(\Pi_{S} z\right)\left[z_{\perp}\right]\right\rangle_{r}\right)_{j \in S} \\
\left(\left\langle J d_{S} \Psi^{\mathrm{nls}}\left(\Pi_{S} z\right)\left[\widehat{z}_{S}\right], \partial_{Y_{j}} d_{\perp} \Psi^{\mathrm{nls}}\left(\Pi_{S} z\right)\left[z_{\perp}\right]\right\rangle_{r}\right)_{j \in S}
\end{array}\right) \\
& +\mathrm{i}\left(\begin{array}{l}
\left(\left\langle\mathbb{J} d_{S}\left(d_{\perp} \Psi^{\mathrm{nls}}\left(\Pi_{S} z\right)\left[z_{\perp}\right]\right)\left[\widehat{z}_{S}\right], \partial_{x_{j}} \Psi^{\mathrm{nls}}\left(\Pi_{S} z\right)\right\rangle_{r}\right)_{j \in S} \\
\left(\left\langle d d_{S}\left(d_{\perp} \Psi^{\mathrm{nls}}\left(\Pi_{S} z\right)\left[z_{\perp}\right]\right)\left[\widehat{z}_{S}\right], \partial_{Y_{j}} \Psi^{\mathrm{nls}}\left(\Pi_{S} z\right)\right\rangle_{r}\right)_{j \in S}
\end{array}\right)
\end{aligned}
$$




$$
+\mathrm{i}\left(\begin{array}{l}
\left(\left\langle\mathbb{d} d_{S}\left(d_{\perp} \Psi^{\mathrm{nls}}\left(\Pi_{S} z\right)\left[z_{\perp}\right]\right)\left[\widehat{z}_{S}\right], \partial_{x_{j}} d_{\perp} \Psi^{\mathrm{nls}}\left(\Pi_{S} z\right)\left[z_{\perp}\right]\right\rangle_{r}\right)_{j \in S} \\
\left(\left\langle\mathbb{J} d_{S}\left(d_{\perp} \Psi^{\mathrm{nls}}\left(\Pi_{S} z\right)\left[z_{\perp}\right]\right)\left[\widehat{z}_{S}\right], \partial_{Y_{j}} d_{\perp} \Psi^{\mathrm{nls}}\left(\Pi_{S} z\right)\left[z_{\perp}\right]\right\rangle_{r}\right)_{j \in S}
\end{array}\right)
$$

and similarly

$$
\begin{aligned}
& L_{S}^{\perp}(z)\left[\widehat{z}_{\perp}\right]:=\mathrm{i}\left(\begin{array}{l}
\left(\left\langle\mathbb{J} d_{\perp} \Psi^{\mathrm{nls}}\left(\Pi_{S} z\right)\left[\widehat{z}_{\perp}\right], \partial_{x_{j}} d_{\perp} \Psi^{\mathrm{nls}}\left(\Pi_{S} z\right)\left[z_{\perp}\right]\right\rangle_{r}\right)_{j \in S} \\
\left(\left\langle\mathbb{J} d_{\perp} \Psi^{\mathrm{nls}}\left(\Pi_{S} z\right)\left[\widehat{z}_{\perp}\right], \partial_{Y_{j}} d_{\perp} \Psi^{\mathrm{nls}}\left(\Pi_{S} z\right)\left[z_{\perp}\right]\right\rangle_{r}\right)_{j \in S}
\end{array}\right), \\
& L_{\perp}^{S}(z)\left[\widehat{z}_{S}\right]:=\mathrm{i}\left(\begin{array}{l}
\left(\left\langle J d_{S}\left(d_{\perp} \Psi^{\mathrm{nls}}\left(\Pi_{S} z\right)\left[z_{\perp}\right]\right)\left[\widehat{z}_{S}\right], \partial_{X_{j}} \Psi^{\mathrm{nls}}\left(\Pi_{S} z\right)\right\rangle_{r}\right)_{j \in S^{\perp}} \\
\left(\left\langle\mathbb{J} d_{S}\left(d_{\perp} \Psi^{\mathrm{nls}}\left(\Pi_{S} z\right)\left[z_{\perp}\right]\right)\left[\widehat{z}_{S}\right], \partial_{y_{j}} \Psi^{\mathrm{nls}}\left(\Pi_{S} z\right)\right\rangle_{r}\right)_{j \in S^{\perp}}
\end{array}\right) .
\end{aligned}
$$

The operator valued map $z \mapsto L(z)$ has the following properties:

Lemma 3.2. The map $L: \mathcal{V} \cap h_{r}^{0} \rightarrow \mathcal{L}\left(h_{c}^{0}, h_{c}^{0}\right), z \mapsto L(z)$ is real analytic. For any $z \in \mathcal{V} \cap h_{r}^{0}$, $\widehat{z} \in h_{C^{\prime}}^{0}$

$$
\|L(z)[\widehat{z}]\|_{0} \lesssim\left\|z_{\perp}\right\|_{0}\|\widehat{z}\|_{0}
$$

and for any $k \in \mathbb{Z}_{\geq 1}, \widehat{z}_{1}, \ldots, \widehat{z}_{k} \in h_{c^{\prime}}^{0}$

$$
\left\|d^{k}(L(z)[\widehat{z}])\left[\widehat{z}_{1}, \ldots, \widehat{z}_{k}\right]\right\|_{0} \lesssim_{k}\|\widehat{z}\|_{0} \prod_{j=1}^{k}\left\|\widehat{z}_{j}\right\|_{0}
$$

Furthermore, the map $L$ is one smoothing, meaning that for any $s \in \mathbb{Z}_{\geq 1}, L: \mathcal{V} \cap h_{r}^{s} \rightarrow$ $\mathcal{L}\left(h_{c}^{0}, h_{c}^{s+1}\right), z \mapsto L(z)$ is real analytic and satisfies the following estimates: for any $z \in$ $\mathcal{V} \cap h_{r}^{s}, \widehat{z} \in h_{c}^{s}$

$$
\|L(z)[\widehat{z}]\|_{s+1} \lesssim_{s}\left\|z_{\perp}\right\|_{s}\|\widehat{z}\|_{0}
$$

and for any $k \in \mathbb{Z}_{\geq 1}, z \in \mathcal{V} \cap h_{r}^{s}, \widehat{z}_{1}, \ldots, \widehat{z}_{k} \in h_{c}^{s}$

$$
\left\|d^{k}(L(z)[\widehat{z}])\left[\widehat{z}_{1}, \ldots, \widehat{z}_{k}\right]\right\|_{s+1} \lesssim_{s, k}\|\widehat{Z}\|_{0} \sum_{j=1}^{k}\left\|\widehat{z}_{j}\right\|_{s} \prod_{i \neq j}\left\|\widehat{Z}_{i}\right\|_{0}+\|\widehat{Z}\|_{0}\left\|z_{\perp}\right\|_{s} \prod_{j=1}^{k}\left\|\widehat{z}_{j}\right\|_{0} .
$$

In particular, $L(z)=0$ for any $z \in \mathcal{V} \cap h_{r}^{0}$ with $z_{\perp}=0$. Finally, $L(z)=-L(z)^{t}$ or, more explicitly, for any $z \in \mathcal{V} \cap h_{r}^{0}$,

$$
L_{S}^{S}(z)^{t}=-L_{S}^{S}(z), \quad L_{S}^{\perp}(z)^{t}=-L_{\perp}^{S}(z), \quad L_{\perp}^{S}(z)^{t}=-L_{S}^{\perp}(z) .
$$


1494 T. Kappeler and R. Montalto

Proof. The analyticity of $L$ follows by Theorem 1.1, using again that $d_{S} d_{\perp} \Psi^{\text {nls }}=$ $d_{S} d_{\perp} B^{\text {nls }}$. Since $\mathbb{J}^{t}=-\mathbb{J}$, one reads off from the expressions (3.41-3.43) that (3.46) holds. The estimates (3.44) and (3.45) follow from Theorem 1.1 by differentiating the expressions in the definitions of $L_{S}^{S}(z), L_{S}^{\perp}(z)$, and $L_{\perp}^{S}(z)$ with respect to $z$.

\section{The Symplectic Corrector $\Psi_{C}$}

In this section we construct the coordinate transformation $\Psi_{C}$ on $\mathcal{V} \cap h_{r}^{0}$ so that the composition $\Psi_{L} \circ \Psi_{C}$ is symplectic. As mentioned in the introduction, we follow Kuksin's scheme of proof in [16], which uses arguments of Moser and Weinstein in the given infinite-dimensional setup. The map $\Psi_{C}$ will be defined as the time-one flow of an appropriately chosen non-autonomous vector field. In the sequel, $\mathcal{V}$ denotes the neighbourhood of $\mathcal{K} \times 0$, given by Propositions 3.1 and 3.2.

For any $z \in \mathcal{V}$ define the following two- and one-forms on $h_{c^{\prime}}^{0}$

$$
\begin{gathered}
\Lambda_{0}:=\Lambda_{M}, \quad \Lambda_{1}(z):=\Psi_{L}^{*} \Lambda(z)=\Lambda_{M}+\Lambda_{L}(z), \\
\lambda_{0}:=\lambda_{M}, \quad \lambda_{1}(z):=\Psi_{L}^{*} \lambda(z) .
\end{gathered}
$$

\subsection{Analysis of the two-form $\Lambda_{1}(z)$}

Note that $d \lambda_{i}=\Lambda_{i}, i=0,1$, and

$$
\Lambda_{1}-\Lambda_{0}=\Lambda_{L}=d\left(\lambda_{1}-\lambda_{0}\right)
$$

In particular, the two-form $\Lambda_{L}$ is closed. By (2.7), (3.39) one has

$$
\Lambda_{1}(z)\left[\widehat{z}, \widehat{z}^{\prime}\right]=\left(\mathcal{L}_{1}(z)[\widehat{z}], \widehat{z}^{\prime}\right)_{r^{\prime}}, \quad \mathcal{L}_{1}(z):=J^{-1}+L(z) .
$$

For any $\tau \in[0,1]$, define the two-form $\Lambda_{\tau}=\Lambda_{\tau}(z)$,

$$
\Lambda_{\tau}:=\tau \Lambda_{1}+(1-\tau) \Lambda_{0},
$$

which can be written as

$$
\Lambda_{\tau}(z)\left[\widehat{z}, \widehat{z}^{\prime}\right]=\left(\mathcal{L}_{\tau}(z)[\widehat{z}], \widehat{z}^{\prime}\right)_{r^{\prime}}, \quad \mathcal{L}_{\tau}(z)=J^{-1}+\tau L(z)
$$

It turns out that for any $\tau \in[0,1]$ and $z \in \mathcal{V} \cap h_{r}^{0}$, the map $\mathcal{L}_{\tau}(z)$ is invertible and one smoothing. More precisely, the following holds:

Lemma 4.1. After shrinking the ball $\mathcal{V}_{\perp} \subset h_{\perp c}^{0}$ in $\mathcal{V}=\mathcal{V}_{S} \times \mathcal{V}_{\perp}$, if necessary, one has that for any $s \in \mathbb{Z}_{\geq 0}, z \in \mathcal{V} \cap h_{r}^{s}$, and $\tau \in[0,1]$, the operator $\mathcal{L}_{\tau}(z): h_{c}^{s} \rightarrow h_{c}^{s}$ is invertible and 
for any $k \in \mathbb{Z}_{\geq 1}, z \in \mathcal{V} \cap h_{r}^{0}, \widehat{z}, \widehat{z}_{1}, \ldots, \widehat{z}_{k} \in h_{c^{\prime}}^{0}$

$$
\left\|\left(\mathcal{L}_{\tau}(z)^{-1}-J\right)[\widehat{z}]\right\|_{0} \lesssim\left\|z_{\perp}\right\|_{0}\|\widehat{z}\|_{0}, \quad\left\|d^{k}\left(\mathcal{L}_{\tau}(z)^{-1}[\widehat{z}]\right)\left[\widehat{z}_{1}, \ldots, \widehat{z}_{k}\right]\right\|_{0} \lesssim_{k}\|\widehat{z}\|_{0} \prod_{j=1}^{k}\left\|\widehat{z}_{j}\right\|_{0}
$$

Moreover for any $s \in \mathbb{Z}_{\geq 1}$ and $\tau \in[0,1]$, the map

$$
\mathcal{L}_{\tau}^{-1}-J: \mathcal{V} \cap h_{r}^{s} \rightarrow \mathcal{L}\left(h_{c^{\prime}}^{s} h_{c}^{s+1}\right), \quad z \mapsto \mathcal{L}_{\tau}(z)^{-1}-J
$$

is real analytic and the following tame estimates hold: for any $k \in \mathbb{Z}_{\geq 1}, z \in \mathcal{V} \cap h_{r}^{s}$, $\widehat{z}, \widehat{z}_{1}, \ldots, \widehat{z}_{k} \in h_{c}^{s}$

$$
\begin{gathered}
\left\|\left(\mathcal{L}_{\tau}(z)^{-1}-J\right)\lceil\widehat{z}]\right\|_{s+1} \lesssim_{s}\left\|z_{\perp}\right\|_{s}\|\widehat{z}\|_{0} \\
\left\|d^{k}\left(\mathcal{L}_{\tau}(z)^{-1}[\widehat{z}]\right)\left[\widehat{z}_{1}, \ldots, \widehat{z}_{k}\right]\right\|_{s+1} \lesssim_{s, k}\|\widehat{z}\|_{0} \sum_{j=1}^{k}\left\|\widehat{z}_{j}\right\|_{s} \prod_{i \neq j}\left\|\widehat{z}_{i}\right\|_{0}+\|\widehat{z}\|_{0}\left\|_{\perp}\right\|_{s} \prod_{j=1}^{k}\left\|\widehat{z}_{j}\right\|_{0} .
\end{gathered}
$$

Proof. For any $\tau \in[0,1]$, we write

$$
\mathcal{L}_{\tau}(z)=J^{-1}\left(\operatorname{Id}+L_{\tau}(z)\right), \quad L_{\tau}(z):=\tau J L(z) .
$$

By (3.40) and Theorem 1.1, the operator $L_{\tau}(z)$ satisfies the estimate $\left\|L_{\tau}(z)[\widehat{z}]\right\|_{0} \leq$ $C_{0}\left\|z_{\perp}\right\|_{0}\|\widehat{z}\|_{0}$, for any $z \in \mathcal{V} \cap h_{r}^{0}$ and $\widehat{z} \in h_{c}^{0}$ for some constant $C_{0}>0$. By shrinking the ball $\mathcal{V}_{\perp}$, if necessary, one has that for any $z_{\perp} \in \mathcal{V}_{\perp}, C_{0}\left\|z_{\perp}\right\|_{0} \leq 1 / 2$, implying that the operator $\mathcal{L}_{\tau}(z)$ is invertible and its inverse $\mathcal{L}_{\tau}(z)^{-1}$ is given by the Neumann series

$$
\mathcal{L}_{\tau}(z)^{-1}=J+\sum_{n \geq 1}(-1)^{n} L_{\tau}(z)^{n} J
$$

By Lemma 3.2, for any $s, n \in \mathbb{Z}_{\geq 1}$ and $\tau \in[0,1]$, one has

$$
\left\|L_{\tau}(z)^{n} J[\widehat{z}]\right\|_{s+1} \leq C(s)\left\|z_{\perp}\right\|_{s}\left\|L_{\tau}(z)^{n-1} J[\widehat{z}]\right\|_{0} \leq C(s)\left(C_{0}\left\|z_{\perp}\right\|_{0}\right)^{n-1}\left\|z_{\perp}\right\|_{s}\|\widehat{z}\|_{0}
$$

for some constant $C(s)>0$. Since $C_{0}\left\|z_{\perp}\right\|_{0} \leq 1 / 2$, one gets

$$
\left\|\left(\mathcal{L}_{\tau}(z)^{-1}-J\right)[\widehat{z}]\right\|_{s+1} \lesssim_{s}\left\|z_{\perp}\right\|_{s}\|\widehat{Z}\|_{0} .
$$

The estimates for the derivatives $d^{k}\left(\mathcal{L}_{\tau}(z)^{-1}[\widehat{z}]\right)$ follow by differentiating the expression (4.6) with respect to $z$ and applying the estimates for $d^{k}(L(z)[\widehat{z}])$ of Lemma 3.2. 
Since by (4.3), the two-form $\Lambda_{L}=\Lambda_{1}-\Lambda_{0}$ is closed and by Lemma 3.2, for any $z \in \mathcal{V} \cap h_{r}^{0}$, $\Lambda_{L}\left(\Pi_{S} z\right)=0$, we can apply Lemma A.1 in Appendix 1. It says that the one-form

$$
\lambda_{L}(z)[\widehat{z}]:=\int_{0}^{1} \Lambda_{L}\left(z_{S}, t z_{\perp}\right)\left[\left(0, z_{\perp}\right),\left(\widehat{z}_{S}, t \widehat{z}_{\perp}\right)\right] \mathrm{d} t
$$

satisfies $d \lambda_{L}=\Lambda_{L}$. By (3.39), (3.40), the one-form $\lambda_{L}(z)$ can be written as

$$
\lambda_{L}(z)[\widehat{z}]=\int_{0}^{1}\left(L\left(z_{S}, t z_{\perp}\right)\left(0, z_{\perp}\right),\left(\widehat{z}_{S}, t \widehat{z}_{\perp}\right)\right)_{r} \mathrm{~d} t=\int_{0}^{1} L_{S}^{\perp}\left(z_{S}, t z_{\perp}\right)\left[z_{\perp}\right] \cdot \widehat{z}_{S} \mathrm{~d} t
$$

Moreover, using that by (3.42), $L_{S}^{\perp}\left(z_{S}, t z_{\perp}\right)=t L_{S}^{\perp}\left(z_{S}, z_{\perp}\right)$, it turns out that

$$
\lambda_{L}(z)[\widehat{z}]=(E(z), \widehat{z})_{r^{\prime}} \quad E(z):=\left(E_{S}(z), 0\right) \in \mathbb{C}^{S} \times \mathbb{C}^{S} \times h_{\perp C^{\prime}}^{0}
$$

where

$$
E_{S}(z):=\frac{1}{2} L_{S}^{\perp}(z)\left[z_{\perp}\right]=\frac{\mathrm{i}}{2}\left(\begin{array}{c}
\left(\left\langle\int \mathrm{d}_{\perp} \Psi^{\mathrm{nls}}\left(\Pi_{S} z\right)\left[z_{\perp}\right], \partial_{X_{j}} \mathrm{~d}_{\perp} \Psi^{\mathrm{nls}}\left(\Pi_{S} z\right)\left[z_{\perp}\right]\right\rangle_{r}\right)_{j \in S} \\
\left(\left\langle\mathbb{J} \mathrm{d}_{\perp} \Psi^{\mathrm{nls}}\left(\Pi_{S} z\right)\left[z_{\perp}\right], \partial_{y_{j}} \mathrm{~d}_{\perp} \Psi^{\mathrm{nls}}\left(\Pi_{S} z\right)\left[z_{\perp}\right]\right\rangle_{r}\right)_{j \in S}
\end{array}\right) .
$$

One of the features of $\lambda_{L}(z)$ is that it is quadratic in $z_{\perp}$. In more detail, we have the following

Lemma 4.2. For any $s \in \mathbb{Z}_{\geq 0}$, the map $E: \mathcal{V} \cap h_{r}^{0} \rightarrow h_{r}^{s}$ is real analytic and satisfies the following tame estimates: for any $z \in \mathcal{V} \cap h_{r}^{0}, \widehat{z} \in h_{c}^{0}$,

$$
\|E(z)\|_{s} \lesssim s\left\|z_{\perp}\right\|_{0}^{2}, \quad\|\mathrm{~d} E(z)[\widehat{z}]\|_{s} \lesssim_{s}\left\|z_{\perp}\right\|_{0}\|\widehat{z}\|_{0},
$$

and any $k \geq 2, \widehat{z}_{1}, \ldots, \widehat{z}_{k} \in h_{c^{\prime}}^{0}$

$$
\left\|\mathrm{d}^{k} E(z)\left[\widehat{z}_{1}, \ldots, \widehat{z}_{k}\right]\right\|_{s} \lesssim_{s, k} \prod_{j=1}^{k}\left\|\widehat{z}_{j}\right\|_{0}
$$

Proof. The lemma follows by the properties of the map $\Psi^{\text {nls }}$, stated in Theorem 1.1, and the fact that $E=\Pi_{S} E,\left\|\Pi_{S} z\right\|_{s} \lesssim_{s}\|z\|_{0}$ for any vector $z \in h_{C}^{0}$, and $\mathcal{V}_{\perp} \subset h_{\perp c}^{0}$ is a ball of radius smaller than 1 . 


\subsection{Outline of the construction of $\Psi_{C}$}

Following arguments of Moser and Weinstein, our candidate for $\Psi_{C}$ is $\Psi_{X}^{0,1}$ where $X \equiv$ $X(z, \tau) \in h_{r}^{0}$ is a non-autonomous vector field with well-defined flow $\Psi_{X}^{\tau_{0}, \tau}, 0 \leq \tau_{0}, \tau \leq 1$, so that $\left(\Psi_{X}^{0,1}\right)^{*} \Lambda_{1}=\Lambda_{0}$. Here $z \in \mathcal{V}$ and the flow is normalized by $\Psi_{X}^{\tau_{0}, \tau_{0}}(z)=z$. To see how to choose $X(z, \tau)$, consider the pullback of the two-form $\Lambda_{\tau}$ by $\Psi_{X}^{0, \tau},\left(\Psi_{X}^{0, \tau}\right)^{*} \Lambda_{\tau}$. Since $\left(\Psi_{X}^{0,0}\right)^{*}=$ $I d$, one has $\left(\Psi_{X}^{0,0}\right)^{*} \Lambda_{0}=\Lambda_{0}$. The desired identity $\left(\Psi_{X}^{0,1}\right)^{*} \Lambda_{1}=\Lambda_{0}$ then follows provided that $\left(\Psi_{X}^{0, \tau}\right)^{*} \Lambda_{\tau}$ is independent of $\tau$, that is, $\partial_{\tau}\left(\left(\Psi_{X}^{0, \tau}\right)^{*} \Lambda_{\tau}\right)=0$. Since $\partial_{\tau} \Lambda_{\tau}=\Lambda_{1}-\Lambda_{0}=d \lambda_{L}$, it turns out that the latter identity holds if $\lambda_{L}+\Lambda_{\tau}[X(\cdot, \tau), \cdot]=0$. When expressed in terms of the bilinear form $(\cdot, \cdot)_{r}$ and taking into account the representation (4.5) of $\Lambda_{\tau}$ and (4.9) of $\lambda_{L}$, the latter identity reads

$$
(E(z), \widehat{z})_{r}+\left(\mathcal{L}_{\tau}(z)[X(z, \tau)], \widehat{z}\right)_{r}=0
$$

We choose the vector field $X(z, \tau)$ so that (4.11) is satisfied.

\subsection{Vector field $X(z, \tau)$ and its flow}

Motivated by (4.11), the non-autonomous vector field $X(z, \tau)$ is defined by

$$
X(z, \tau):=-\mathcal{L}_{\tau}(z)^{-1} E(z), \quad z \in \mathcal{V}_{S} \times \mathcal{V}_{\perp}, \quad \tau \in[0,1]
$$

Lemmata 4.1 and 4.2 lead to the following

Lemma 4.3. The vector field $X:\left(\mathcal{V} \cap h_{r}^{0}\right) \times[0,1] \rightarrow h_{r}^{0}$ is real analytic and one smoothing, meaning that for any $s \in \mathbb{Z}_{\geq 1}$

$$
X:\left(\mathcal{V} \cap h_{r}^{s}\right) \times[0,1] \rightarrow h_{r}^{s+1}
$$

is real analytic. In addition, the following tame estimates hold: for any $\tau \in[0,1], z \in$ $\mathcal{V} \cap h_{r}^{0}, \widehat{z} \in h_{c^{\prime}}^{0}$

$$
\|X(z, \tau)\|_{0} \lesssim\left\|z_{\perp}\right\|_{0}^{2}, \quad\|\mathrm{~d} X(z, \tau)[\widehat{z}]\|_{0} \lesssim\left\|z_{\perp}\right\|_{0}\|\widehat{z}\|_{0}
$$

and for any $k \geq 2, \widehat{z}_{1}, \ldots, \widehat{z}_{k} \in h_{c^{\prime}}^{0}$

$$
\left\|\mathrm{d}^{k} X(\boldsymbol{z}, \tau)\left[\widehat{z}_{1}, \ldots, \widehat{z}_{k}\right]\right\|_{0} \lesssim_{k} \prod_{j=1}^{k}\left\|\widehat{z}_{j}\right\|_{0}
$$


Moreover, for any $s \in \mathbb{Z}_{\geq 1}, z \in \mathcal{V} \cap h_{r}^{s}, \widehat{z} \in h_{c^{\prime}}^{s}$

$$
\|X(z, \tau)\|_{s+1} \lesssim_{s}\left\|z_{\perp}\right\|_{s}\left\|z_{\perp}\right\|_{0}, \quad\|\mathrm{~d} X(z, \tau)[\widehat{z}]\|_{s+1} \lesssim_{s}\left\|z_{\perp}\right\|_{0}\|\widehat{Z}\|_{s}+\left\|z_{\perp}\right\|_{s}\|\widehat{Z}\|_{0}
$$

and for any $k \geq 2, \widehat{z}_{1}, \ldots, \widehat{z}_{k} \in h_{c^{\prime}}^{s}$

$$
\left\|\mathrm{d}^{k} X(z, \tau)\left[\widehat{Z}_{1}, \ldots, \widehat{Z}_{k}\right]\right\|_{s+1} \lesssim s, k \sum_{j=1}^{k}\left\|\widehat{Z}_{j}\right\|_{s} \prod_{i \neq j}\left\|\widehat{Z}_{i}\right\|_{0}+\left\|z_{\perp}\right\|_{s} \prod_{j=1}^{k}\left\|\widehat{Z}_{j}\right\|_{0}
$$

Proof. The lemma follows from Lemmata 4.1 and 4.2 .

We now want to study the flow of the non-autonomous differential equation

$$
\partial_{\tau} z=X(z, \tau)
$$

Recall that for any $r>0$, we denote by $\mathcal{V}_{\perp}(r)$ the ball in $h_{\perp c}^{0}$ of radius $r$, centred at 0 , and for any $\tau_{0}, \tau \in[0,1]$ by $\Psi_{X}^{\tau_{0}, \tau}$ the flow map of the differential equation (4.15), satisfying $\Psi_{X}^{\tau_{0}, \tau_{0}}(z)=z$. By a standard contraction argument, there exists an open neighbourhood $\mathcal{V}_{S}^{\prime} \subseteq \mathcal{V}_{S}$ of $\mathcal{K}$ in $\mathbb{C}^{S} \times \mathbb{C}^{S}$ and $\delta>0$ with $\mathcal{V}_{\perp}(2 \delta) \subset \mathcal{V}_{\perp}$ such that for any $\tau, \tau_{0} \in[0,1]$

$$
\Psi_{X}^{\tau_{0}, \tau}: \mathcal{V}_{\delta}^{\prime} \cap h_{r}^{0} \rightarrow \mathcal{V}_{2 \delta} \cap h_{r}^{0}, \quad \mathcal{V}_{\delta}^{\prime}:=\mathcal{V}_{S}^{\prime} \times \mathcal{V}_{\perp}(\delta), \quad \mathcal{V}_{2 \delta}:=\mathcal{V}_{S} \times \mathcal{V}_{\perp}(2 \delta)
$$

is well defined and real analytic. In the next lemma we state the smoothing estimates for $\Psi_{X}^{\tau_{0}, \tau}-\iota d$ where $\iota d$ denotes the identity map on $\mathcal{V}_{\delta}^{\prime} \cap h_{r}^{0}$.

Lemma 4.4. By choosing $0<\delta<1$ smaller, if necessary, it follows that for any $\tau$, $\tau_{0} \in$ $[0,1]$, the map $\Psi_{X}^{\tau_{0}, \tau}-\iota d: \mathcal{V}_{\delta}^{\prime} \cap h_{r}^{0} \rightarrow h_{r}^{0}$ is one smoothing, meaning that for any $s \in \mathbb{Z}_{\geq 1}$ ， the map

$$
\Psi_{X}^{\tau_{0}, \tau}-\iota d: \mathcal{V}_{\delta}^{\prime} \cap h_{r}^{s} \rightarrow h_{r}^{s+1}
$$

is real analytic. Furthermore, the following tame estimates hold: for any $z \in \mathcal{V}_{\delta}^{\prime} \cap h_{r}^{0}$, $\widehat{z} \in h_{c^{\prime}}^{0}$

$$
\left\|\Psi_{X}^{\tau_{0}, \tau}(z)-z\right\|_{0} \lesssim\left\|z_{\perp}\right\|_{0}^{2}, \quad\left\|\left(\mathrm{~d} \Psi_{X}^{\tau_{0}, \tau}(z)-\mathrm{Id}\right)[\widehat{z}]\right\|_{0} \lesssim\left\|z_{\perp}\right\|_{0}\|\widehat{z}\|_{0}
$$

and for any $k \geq 2, \widehat{z}_{1}, \ldots, \widehat{z}_{k} \in h_{c^{\prime}}^{0}$

$$
\left\|\mathrm{d}^{k} \Psi_{X}^{\tau_{0}, \tau}(z)\left[\widehat{Z}_{1}, \ldots, \widehat{z}_{k}\right]\right\|_{0} \lesssim_{k} \prod_{j=1}^{k}\left\|\widehat{z}_{j}\right\|_{0}
$$


whereas for any $s \in \mathbb{Z}_{\geq 1}, z \in \mathcal{V}_{\delta}^{\prime} \cap h_{r}^{s}, \widehat{z} \in h_{c^{\prime}}^{s}$

$$
\left\|\Psi_{X}^{\tau_{0}, \tau}(z)-z\right\|_{s+1} \lesssim_{s}\left\|z_{\perp}\right\|_{s}\left\|z_{\perp}\right\|_{0}, \quad\left\|\left(\mathrm{~d} \Psi_{X}^{\tau_{0}, \tau}(z)-\mathrm{Id}\right)[\widehat{z}]\right\|_{s+1} \lesssim_{s}\left\|z_{\perp}\right\|_{0}\|\widehat{Z}\|_{s}+\left\|z_{\perp}\right\|_{s}\|\widehat{Z}\|_{0}
$$

and for any $k \geq 2, \widehat{z}_{1}, \ldots, \widehat{z}_{k} \in h_{c^{\prime}}^{s}$

$$
\left\|\mathrm{d}^{k} \Psi_{X}^{\tau_{0}, \tau}(z)\left[\widehat{z}_{1}, \ldots, \widehat{z}_{k}\right]\right\|_{s+1} \lesssim s, k \sum_{j=1}^{k}\left\|\widehat{z}_{j}\right\|_{s} \prod_{i \neq j}\left\|\widehat{z}_{i}\right\|_{0}+\left\|z_{\perp}\right\|_{s} \prod_{j=1}^{k}\left\|\widehat{z}_{j}\right\|_{0} .
$$

Proof. For any $\tau_{0}, \tau \in[0,1]$ and $z \in \mathcal{V}_{\delta}^{\prime} \cap h_{r}^{0}$, the flow $\Psi_{X}^{\tau_{0}, \tau}(z)$ satisfies the integral equation

$$
\Psi_{X}^{\tau_{0}, \tau}(z)=z+\int_{\tau_{0}}^{\tau} X\left(\Psi_{X}^{\tau_{0}, t}(z), t\right) \mathrm{d} t
$$

In view of the estimate (4.14) of the vector field $X(z, \tau)$, we first estimate $\left\|\Pi_{\perp} \Psi_{X}^{\tau_{0}, \tau}(z)\right\|_{S}$ for $z \in \mathcal{V}_{\delta}^{\prime} \cap h_{r}^{s}$ with $s \in \mathbb{Z}_{\geq 0}$. Applying the operator $\Pi_{\perp}$ to both sides of the identity (4.19), one gets

$$
\Pi_{\perp} \Psi_{X}^{\tau_{0}, \tau}(z)=\Pi_{\perp} Z+\int_{\tau_{0}}^{\tau} \Pi_{\perp} X\left(\Psi_{X}^{\tau_{0}, t}(z), t\right) \mathrm{d} t
$$

By Lemma 4.3, for any $\tau, \tau_{0} \in[0,1]$, one has

$$
\left\|\Pi_{\perp} \Psi_{X}^{\tau_{0}, \tau}(z)\right\|_{s} \leq\left\|z_{\perp}\right\|_{s}+C(s)\left|\int_{\tau_{0}}^{\tau}\left\|\Pi_{\perp} \Psi_{X}^{\tau_{0}, t}(z)\right\|_{s}\left\|\Pi_{\perp} \Psi_{X}^{\tau_{0}, t}(z)\right\|_{0} \mathrm{~d} t\right|
$$

for some constant $C(s)>0$, only depending on $s$. Then by shrinking $\delta>0$, if necessary, so that for $z_{\perp} \in \mathcal{V}_{\perp}(\delta)$, we have $\sup _{\tau_{0, \tau \in[0,1]}}\left\|\Pi_{\perp} \Psi_{X}^{\tau_{0}, \tau}(z)\right\|_{0} \leq 1$, the above estimate becomes

$$
\left\|\Pi_{\perp} \Psi_{X}^{\tau_{0}, \tau}(z)\right\|_{s} \leq\left\|z_{\perp}\right\|_{s}+C(s)\left|\int_{\tau_{0}}^{\tau}\left\|\Pi_{\perp} \Psi_{X}^{\tau_{0}, t}(z)\right\|_{s} \mathrm{~d} t\right| .
$$

By the Gronwall inequality one then gets

$$
\sup _{\tau_{0, \tau \in[0,1]}}\left\|\Pi_{\perp} \Psi_{X}^{\tau_{0}, \tau}(z)\right\|_{s} \lesssim_{s}\left\|z_{\perp}\right\|_{s}, \quad \forall z \in \mathcal{V}_{\delta}^{\prime} \cap h_{r}^{s}
$$

Now let us prove (4.18). By (4.19), using again Lemma 4.3, one gets for any $s \in \mathbb{Z}_{\geq 1}$, $\tau_{0}, \tau \in[0,1]$, and $z \in \mathcal{V}_{\delta}^{\prime} \cap h_{r}^{s}$

$$
\begin{aligned}
\left\|\Psi_{X}^{\tau_{0}, \tau}(z)-z\right\|_{s+1} & \leq\left|\int_{\tau_{0}}^{\tau}\left\|X\left(\Psi_{X}^{\tau_{0}, t}(z), t\right)\right\|_{s+1} \mathrm{~d} t\right| \lesssim \sup _{t \in[0,1]}\left\|\Pi_{\perp} \Psi_{X}^{\tau_{0}, t}(z)\right\|_{s} \sup _{t \in[0,1]}\left\|\Pi_{\perp} \Psi_{X}^{\tau_{0}, t}(z)\right\|_{0} \\
& \stackrel{(4.22)}{\lesssim}\left\|z_{\perp}\right\|_{s}\left\|z_{\perp}\right\|_{0}
\end{aligned}
$$


which is the first claimed inequality in (4.18). To prove the one for the differential $d \Psi_{X}^{\tau_{0}, \tau}-$ Id, differentiate (4.19) with respect to $z$. Using the chain rule one gets

$$
\mathrm{d} \Psi_{X}^{\tau_{0}, \tau}(z)[\widehat{Z}]=\widehat{z}+\int_{\tau_{0}}^{\tau} \mathrm{d} X\left(\Psi_{X}^{\tau_{0}, t}(z), t\right)\left[\mathrm{d} \Psi_{X}^{\tau_{0}, t}(z)[\widehat{z}]\right] \mathrm{d} t .
$$

By applying the estimates of $\mathrm{d} X(\cdot, \tau)$ of Lemma 4.3, it follows that for any $s \in \mathbb{Z}_{\geq 0}$ there is a constant $C(s)>0$ such that

$$
\begin{aligned}
& \left\|\mathrm{d} \Psi_{X}^{\tau_{0}, \tau}(z)[\widehat{z}]\right\|_{s} \\
& \quad \leq\|\widehat{z}\|_{s}+C(s)\left|\int_{\tau_{0}}^{\tau}\left(\left\|\Pi_{\perp} \Psi_{X}^{\tau_{0}, t}(z)\right\|_{s}\left\|\mathrm{~d} \Psi_{X}^{\tau_{0}, t}(z)[\widehat{z}]\right\|_{0}+\left\|\Pi_{\perp} \Psi_{X}^{\tau_{0}, t}(z)\right\|_{0}\left\|\mathrm{~d} \Psi_{X}^{\tau_{0}, t}(z)[\widehat{z}]\right\|_{s}\right) \mathrm{d} t\right| \\
& \quad \stackrel{(4.22)}{\leq}\|\widehat{z}\|_{s}+C_{1}(s)\left|\int_{\tau_{0}}^{\tau}\left(\left\|z_{\perp}\right\|_{s}\left\|\mathrm{~d} \Psi_{X}^{\tau_{0}, t}(z)[\widehat{z}]\right\|_{0}+\left\|z_{\perp}\right\|_{0}\left\|\mathrm{~d} \Psi_{X}^{\tau_{0}, t}(z)[\widehat{z}]\right\|_{s}\right) \mathrm{d} t\right| .
\end{aligned}
$$

for some constant $C_{1}(s)>C(s)>0$. For $s=0$, using that $\left\|z_{\perp}\right\|_{0} \leq \delta<1$, (4.25) becomes

$$
\left\|\mathrm{d} \Psi_{X}^{\tau_{0}, \tau}(z)[\widehat{z}]\right\|_{0} \leq\|\widehat{Z}\|_{0}+2 C_{1}(0)\left|\int_{\tau_{0}}^{\tau}\left\|\mathrm{d} \Psi_{X}^{\tau_{0}, t}(z)[\widehat{z}]\right\|_{0} \mathrm{~d} t\right|
$$

and hence by the Gronwall inequality

$$
\left\|\mathrm{d} \Psi_{X}^{\tau_{0}, \tau}(z)[\widehat{Z}]\right\|_{0} \lesssim\|\widehat{Z}\|_{0}
$$

For $s \in \mathbb{Z}_{\geq 1}$, substitute the latter estimate into (4.25) to get, again using that $\left\|z_{\perp}\right\|_{0}<\delta<1$

$$
\left\|\mathrm{d} \Psi_{X}^{\tau_{0}, \tau}(z)[\widehat{Z}]\right\|_{s} \leq\|\widehat{Z}\|_{s}+C_{2}(s)\left\|z_{\perp}\right\|_{s}\|\widehat{Z}\|_{0}+C_{2}(s)\left|\int_{\tau_{0}}^{\tau}\left\|\mathrm{d} \Psi_{X}^{\tau_{0}, t}(z)[\widehat{Z}]\right\|_{s} \mathrm{~d} t\right|
$$

for some constant $C_{2}(s)>C_{1}(s)$. Then using again the Gronwall inequality one concludes that for any $0 \leq \tau_{0} \leq 1$,

$$
\sup _{\tau \in[0,1]}\left\|\mathrm{d} \Psi_{X}^{\tau_{0}, \tau}(z)[\widehat{Z}]\right\|_{s} \lesssim s\|\widehat{Z}\|_{s}+\left\|z_{\perp}\right\|_{s}\|\widehat{Z}\|_{0}
$$

We are now ready to prove the second estimate in (4.18). By (4.24) and the smoothing estimates on $\mathrm{d} X(\cdot, \tau)$ of Lemma 4.3 , one gets that for any $s \in \mathbb{Z}_{\geq 1}, 0 \leq \tau_{0} \leq 1$,

$$
\begin{gathered}
\left\|\left(\mathrm{d} \Psi_{X}^{\tau_{0}, \tau}(z)-\mathrm{Id}\right)[\widehat{z}]\right\|_{s+1} \lesssim_{s} \sup _{t \in[0,1]}\left\|\Pi_{\perp} \Psi_{X}^{\tau_{0}, t}(z)\right\|_{s} \sup _{t \in[0,1]}\left\|\mathrm{d} \Psi_{X}^{\tau_{0}, t}(z)[\widehat{z}]\right\|_{0} \\
\quad+\sup _{t \in[0,1]}\left\|\Pi_{\perp} \Psi_{X}^{\tau_{0}, t}(z)\right\|_{0} \sup _{t \in[0,1]}\left\|\mathrm{d} \Psi_{X}^{\tau_{0}, t}(z)[\widehat{z}]\right\|_{s} \\
\stackrel{(4.22),(4.27)}{\lesssim s_{S}}\left\|z_{\perp}\right\|_{s}\|\widehat{Z}\|_{0}+\left\|z_{\perp}\right\|_{0}\|\widehat{Z}\|_{s}
\end{gathered}
$$


where we used again that $\left\|z_{\perp}\right\|_{0}<\delta<1$. Hence the claimed estimate for $\mathrm{d} \Psi_{X}^{\tau_{0}, \tau}(z)-\operatorname{Id}$ in (4.18) is established. The estimates for the higher-order derivatives $\mathrm{d}^{k} \Psi_{X}^{\tau_{0}, \tau}, k \geq 2$, follow by similar arguments, differentiating $k$-times the equation (4.19) with respect to $z$.

\subsection{Definition of $\Psi_{C}$ and its properties}

Our candidate for the symplectic corrector is the time-one flow map of $X(z, \tau)$,

$$
\Psi_{C}:=\Psi_{X}^{0,1}: \mathcal{V}_{\delta}^{\prime} \cap h_{r}^{0} \rightarrow h_{r}^{0}
$$

Clearly, $\Psi_{C}$ is one to one and its inverse is given by the backward flow of the PDE (4.15), namely $\Psi_{C}^{-1}=\Psi_{X}^{1,0}$. Hence the maps $\Psi_{C}^{ \pm 1}$ satisfy the estimates stated in Lemma 4.4. Furthermore, recall that for any $\tau \in[0,1]$, the two-form $\Lambda_{\tau}$ admits the representation (4.5). Then the following Darboux lemma holds.

Proposition 4.1. The map $\Psi_{C}$ is a symplectic corrector, that is, for any $z \in \mathcal{V}_{\delta}^{\prime} \cap h_{r}^{0}$, $\Psi_{C}^{*} \Lambda_{1}(z)=\Lambda_{0}$.

Proof. For any $\tau \in[0,1]$, consider the two-form $\left(\Psi_{X}^{0, \tau}\right)^{*} \Lambda_{\tau}$. Since $\Psi_{X}^{0,0}=\operatorname{Id}$, one has $\left(\Psi_{X}^{0,0}\right)^{*} \Lambda_{0}=\Lambda_{0}$ and hence it suffices to prove that the map $\tau \mapsto\left(\Psi_{X}^{0, \tau}\right)^{*} \Lambda_{\tau}$ is constant or, equivalently,

$$
\partial_{\tau}\left(\left(\Psi_{X}^{0, \tau}\right)^{*} \Lambda_{\tau}\right)=0, \quad \forall \tau \in[0,1]
$$

By Cartan's identity (see, e.g., Lemma 1.2 in [16]) and the fact that $\Lambda_{\tau}$ is closed, it follows that

$$
\partial_{\tau}\left(\left(\Psi_{X}^{0, \tau}\right)^{*} \Lambda_{\tau}\right)=\left(\Psi_{X}^{0, \tau}\right)^{*}\left(\partial_{\tau} \Lambda_{\tau}+\mathrm{d}\left(\Lambda_{\tau}[X(\cdot, \tau), \cdot]\right)\right) .
$$

Since $\partial_{\tau} \Lambda_{\tau} \stackrel{(4.4)}{=} \Lambda_{1}-\Lambda_{0}=\Lambda_{L}$ and $\Lambda_{L} \stackrel{(4.8)}{=} \mathrm{d} \lambda_{L}$, it remains to prove that

$$
\mathrm{d}\left(\lambda_{L}+\Lambda_{\tau}[X(\cdot, \tau), \cdot]\right)=0
$$

By (4.5), (4.9), (4.12), one has for any $\tau \in[0,1], z \in \mathcal{V}_{\delta}^{\prime} \cap h_{r}^{0}$, and $\widehat{z} \in h_{c}^{0}$

$$
\lambda_{L}(z)[\widehat{z}]+\Lambda_{\tau}[X(z, \tau), \widehat{z}]=(E(z), \widehat{z})_{r}-\left(\mathcal{L}_{\tau}(z) \mathcal{L}_{\tau}(z)^{-1} E(z), \widehat{z}\right)_{r}=0 .
$$

It means that

$$
\lambda_{L}+\Lambda_{\tau}[X(\cdot, \tau), \cdot]=0, \quad \forall \tau \in[0,1]
$$

proving the proposition. 
As a consequence of Lemma 4.4 we get the following.

Corollary 4.1. (i) For any $s \in \mathbb{Z}_{\geq 0}$, the map $\Psi_{C}: \mathcal{V}_{\delta}^{\prime} \cap h_{r}^{s} \rightarrow h_{r}^{s}$ is a real analytic diffeomorphism onto its image and its nonlinear part is one smoothing, meaning that for any $s \in \mathbb{Z}_{\geq 1}$, the map $B_{C}:=\Psi_{C}-\iota d: \mathcal{V}_{\delta}^{\prime} \cap h_{r}^{s} \rightarrow h_{r}^{s+1}$ is real analytic. Furthermore, $B_{C}$ satisfies the following tame estimates: for any $z \in \mathcal{V}_{\delta}^{\prime} \cap h_{r}^{0}, \widehat{z} \in h_{c}^{0}$,

$$
\left\|B_{C}(z)\right\|_{0} \lesssim\left\|z_{\perp}\right\|_{0}^{2}, \quad\left\|d B_{C}(z)[\widehat{z}]\right\|_{0} \lesssim\left\|z_{\perp}\right\|_{0}\|\widehat{z}\|_{0}
$$

and for any $k \geq 2, \widehat{z}_{1}, \ldots, \widehat{z}_{k} \in h_{c}^{0}$

$$
\left\|\mathrm{d}^{k} B_{C}(z)\left[\widehat{z}_{1}, \ldots, \widehat{z}_{k}\right]\right\|_{0} \lesssim_{k} \prod_{j=1}^{k}\left\|\widehat{Z}_{j}\right\|_{0}
$$

whereas for any $s \in \mathbb{Z}_{\geq 1}, z \in \mathcal{V}_{\delta}^{\prime} \cap h_{r}^{s}, \widehat{z} \in h_{c^{\prime}}^{s}$

$$
\left\|B_{C}(z)\right\|_{s+1} \lesssim_{s}\left\|z_{\perp}\right\|_{s}\left\|z_{\perp}\right\|_{0}, \quad\left\|\mathrm{~d} B_{C}(z)[\widehat{z}]\right\|_{s+1} \lesssim_{s}\left\|z_{\perp}\right\|_{0}\|\widehat{z}\|_{s}+\left\|z_{\perp}\right\|_{s}\|\widehat{z}\|_{0}
$$

and for any $k \geq 2, \widehat{z}, \widehat{z}_{1}, \ldots, \widehat{z}_{k} \in h_{c}^{s}$,

$$
\left\|d^{k} B_{C}(z)\left[\widehat{z}_{1}, \ldots, \widehat{z}_{k}\right]\right\|_{s+1} \lesssim_{s, k} \sum_{j=1}^{k}\left\|\widehat{z}_{j}\right\|_{s} \prod_{i \neq j}\left\|\widehat{z}_{i}\right\|_{0}+\left\|z_{\perp}\right\|_{s} \prod_{j=1}^{k}\left\|\widehat{z}_{j}\right\|_{0} .
$$

(ii) The map $A_{C}:=\Psi_{C}^{-1}-\iota d: \Psi_{C}\left(\mathcal{V}_{\delta}^{\prime}\right) \cap h_{r}^{0} \rightarrow h_{r}^{0}$ is real analytic and satisfies the following tame estimates: for any $z \in \Psi_{C}\left(\mathcal{V}_{\delta}^{\prime}\right) \cap h_{r}^{0}, \widehat{z} \in h_{c^{\prime}}^{0}$

$$
\left\|A_{C}(z)\right\|_{0} \lesssim\left\|z_{\perp}\right\|_{0}^{2}, \quad\left\|\mathrm{~d} A_{C}(z)[\widehat{z}]\right\|_{0} \lesssim\left\|z_{\perp}\right\|_{0}\|\widehat{z}\|_{0}
$$

and for any $k \geq 2, \widehat{z}_{1}, \ldots, \widehat{z}_{k} \in h_{c}^{0}$,

$$
\left\|\mathrm{d}^{k} A_{C}(z)\left[\widehat{z}_{1}, \ldots, \widehat{z}_{k}\right]\right\|_{0} \lesssim k \prod_{j=1}^{k}\left\|\widehat{Z}_{j}\right\|_{0}
$$

Furthermore, for any $s \in \mathbb{Z}_{\geq 1}, A_{C}: \Psi_{C}\left(\mathcal{V}_{\delta}^{\prime}\right) \cap h_{r}^{s} \rightarrow h_{r}^{s+1}$ is real analytic and satisfies the following tame estimates: for any $z \in \Psi_{C}\left(\mathcal{V}_{\delta}^{\prime}\right) \cap h_{r}^{s}, \widehat{z} \in h_{c^{\prime}}^{s}$,

$$
\left\|A_{C}(z)\right\|_{s+1} \lesssim_{s}\left\|z_{\perp}\right\|_{s}\left\|z_{\perp}\right\|_{0}, \quad\left\|\mathrm{~d} A_{C}(z)[\widehat{Z}]\right\|_{s+1} \lesssim_{s}\left\|z_{\perp}\right\|_{0}\|\widehat{Z}\|_{s}+\left\|z_{\perp}\right\|_{s}\|\widehat{Z}\|_{0}
$$


and for any $k \geq 2, \widehat{z}_{1}, \ldots, \widehat{z}_{k} \in h_{c^{\prime}}^{s}$

$$
\left\|\mathrm{d}^{k} A_{C}(z)\left[\widehat{z}_{1}, \ldots, \widehat{z}_{k}\right]\right\|_{s+1} \lesssim_{s, k} \sum_{j=1}^{k}\left\|\widehat{z}_{j}\right\|_{s} \prod_{i \neq j}\left\|\widehat{z}_{i}\right\|_{0}+\left\|z_{\perp}\right\|_{s} \prod_{j=1}^{k}\left\|\widehat{z}_{j}\right\|_{0} .
$$

Proof. The claimed results are a special case of Lemma 4.4, since $\Psi_{C}=\Psi_{X}^{0,1}$ and $\Psi_{C}^{-1}=$ $\Psi_{X}^{1,0}$.

An immediate consequence of Corollary 4.1 is the following result, needed in Section 5.2.

Corollary 4.2. The Taylor expansion of the map $B_{C}=\Psi_{C}-\iota d$ around $\Pi_{S} z$ up to order three is of the form

$$
B_{C}(z)=B_{2}^{C}(z)+B_{3}^{C}(z), \quad z \in \mathcal{V}_{\delta}^{\prime} \cap h_{r}^{0}
$$

where

$$
B_{2}^{C}(z):=\frac{1}{2} \mathrm{~d}^{2} B_{C}\left(\Pi_{S} z\right)\left[\Pi_{\perp} z, \Pi_{\perp} z\right]
$$

and $B_{3}^{C}(z)$ is the Taylor remainder term

$$
B_{3}^{C}(z):=\frac{1}{2} \int_{0}^{1}(1-t)^{2} \mathrm{~d}^{3} B_{C}\left(\Pi_{S} z+t \Pi_{\perp} z\right)\left[\Pi_{\perp} z, \Pi_{\perp} z, \Pi_{\perp} z\right] \mathrm{d} t .
$$

The maps $B_{i}^{C}: \mathcal{V}_{\delta}^{\prime} \cap h_{r}^{0} \rightarrow h_{r}^{0}, i=2,3$, are real analytic and $B_{3}^{C}$ satisfies the following estimates: for any $z \in \mathcal{V}_{\delta}^{\prime} \cap h_{r}^{0}, \widehat{z}_{,}, \widehat{z}_{1}, \widehat{z}_{2} \in h_{c^{\prime}}^{0}$

$$
\left\|B_{3}^{C}(z)\right\|_{0} \lesssim\left\|z_{\perp}\right\|_{0}^{3}, \quad\left\|\mathrm{~d} B_{3}^{C}(z)[\widehat{z}]\right\|_{0} \lesssim\left\|z_{\perp}\right\|_{0}^{2}\|\widehat{z}\|_{0}, \quad\left\|\mathrm{~d}^{2} B_{3}^{C}(z)\left[\widehat{z}_{1}, \widehat{z}_{2}\right]\right\|_{0} \lesssim\left\|z_{\perp}\right\|_{0}\left\|\widehat{z}_{1}\right\|_{0}\left\|\widehat{z}_{2}\right\|_{0}
$$

and for any $k \geq 3, \widehat{z}_{1}, \ldots, \widehat{z}_{k} \in h_{c^{\prime}}^{0}$

$$
\left\|\mathrm{d}^{k} B_{3}^{C}(z)\left[\widehat{z}_{1}, \ldots, \widehat{z}_{k}\right]\right\|_{0} \lesssim_{k} \prod_{j=1}^{k}\left\|\widehat{z}_{j}\right\|_{0}
$$

Furthermore, for any $s \in \mathbb{Z}_{\geq 1}, B_{i}^{C}: \mathcal{V}_{\delta}^{\prime} \cap h_{r}^{s} \rightarrow h_{r}^{s+1}, i=2,3$, are real analytic and $B_{3}^{C}$ satisfies the following tame estimates: for any $z \in \mathcal{V}_{\delta}^{\prime} \cap h_{r}^{s}, \widehat{z}, \widehat{z}_{1}, \widehat{z}_{2} \in h_{c^{\prime}}^{s}$

$$
\begin{gathered}
\left\|B_{3}^{C}(z)\right\|_{s+1} \lesssim_{s}\left\|z_{\perp}\right\|_{s}\left\|z_{\perp}\right\|_{0}^{2}, \quad\left\|\mathrm{~d} B_{3}^{C}(z)[\widehat{z}]\right\|_{s+1} \lesssim_{s}\left\|z_{\perp}\right\|_{0}^{2}\|\widehat{z}\|_{s}+\left\|z_{\perp}\right\|_{s}\left\|z_{\perp}\right\|_{0}\|\widehat{z}\|_{0}, \\
\left\|\mathrm{~d}^{2} B_{3}^{C}(z)\left[\widehat{z}_{1}, \widehat{z}_{2}\right]\right\|_{s+1} \lesssim_{s}\left\|z_{\perp}\right\|_{0}\left(\left\|\widehat{z}_{1}\right\|_{0}\left\|\widehat{z}_{2}\right\|_{s}+\left\|\widehat{z}_{1}\right\|_{s}\left\|\widehat{z}_{2}\right\|_{0}\right)+\left\|z_{\perp}\right\|_{s}\left\|\widehat{z}_{1}\right\|_{0}\left\|\widehat{z}_{2}\right\|_{0}
\end{gathered}
$$


and for any $k \geq 3, \widehat{z}, \widehat{z}_{1}, \ldots, \widehat{z}_{k} \in h_{c^{\prime}}^{s}$

$$
\left\|\mathrm{d}^{k} B_{3}^{C}(z)\left[\widehat{Z}_{1}, \ldots, \widehat{\boldsymbol{Z}}_{k}\right]\right\|_{s+1} \lesssim_{s, k} \sum_{j=1}^{k}\left\|\widehat{\boldsymbol{Z}}_{j}\right\|_{s} \prod_{i \neq j}\left\|\widehat{\boldsymbol{Z}}_{i}\right\|_{0}+\left\|\boldsymbol{z}_{\perp}\right\|_{s} \prod_{j=1}^{k}\left\|\widehat{\boldsymbol{Z}}_{j}\right\|_{0}
$$

Proof. By Corollary 4.1, $B_{C}\left(\Pi_{S} z\right)=0$ and $\mathrm{d} B_{C}\left(\Pi_{S} z\right)=0$. Thus $B_{C}(z)=B_{2}^{C}(z)+B_{3}^{C}(z)$ is the Taylor expansion of $B_{C}$ around $\Pi_{S} z$ with Taylor remainder term given by (4.30). The claimed analyticity and tame estimates follow from Corollary 4.1.

\section{Proof of Theorem 1.2}

In this section we prove Theorem 1.2. First we introduce and discuss our new canonical coordinates and then express the Hamiltonian of the dNLS equation in the new coordinates.

\subsection{New canonical coordinates}

Our candidate of the canonical transformation is the map

$$
\Psi:=\Psi_{L} \circ \Psi_{C}: \mathcal{V}_{\delta}^{\prime} \rightarrow H_{C}^{0},
$$

where $\mathcal{V}_{\delta}^{\prime}$ is the neighbourhood introduced in (4.16).

Proposition 5.1. By shrinking $0<\delta<1$, if necessary, it follows that for any $s \in \mathbb{Z}_{\geq 0}$, $\Psi: \mathcal{V}_{\delta}^{\prime} \cap h_{r}^{s} \rightarrow H_{r}^{s}$ is a real analytic symplectic diffeomorphism onto its image with the property that its nonlinear part $B:=\Psi-F_{\mathrm{nls}}^{-1}: \mathcal{V}_{\delta}^{\prime} \cap h_{r}^{0} \rightarrow H_{r}^{0}$ satisfies the following estimates: for any $k \in \mathbb{Z}_{\geq 1}, z \in \mathcal{V}_{\delta}^{\prime} \cap h_{r}^{0}, \widehat{z}_{1}, \ldots, \widehat{z}_{k} \in h_{c}^{0}$,

$$
\|B(z)\|_{0} \lesssim 1, \quad\left\|\mathrm{~d}^{k} B(z)\left[\widehat{z}_{1}, \ldots, \widehat{z}_{k}\right]\right\|_{0} \lesssim k \prod_{j=1}^{k}\left\|\widehat{z}_{j}\right\|_{0}
$$

Furthermore, $B$ is one smoothing, meaning that for any $s \in \mathbb{Z}_{\geq 1}$, the map $B: \mathcal{V}_{\delta}^{\prime} \cap h_{r}^{s} \rightarrow H_{r}^{s+1}$ is real analytic, and it satisfies the following tame estimates: for any $k \in \mathbb{Z}_{\geq 1}, z \in \mathcal{V}_{\delta}^{\prime} \cap h_{r}^{s}$, and $\widehat{z}_{1}, \ldots, \widehat{z}_{k} \in h_{c}^{s}$,

$$
\|B(z)\|_{s+1} \lesssim_{s} 1+\left\|z_{\perp}\right\|_{s}, \quad\left\|\mathrm{~d}^{k} B(z)\left[\widehat{z}_{1}, \ldots, \widehat{z}_{k}\right]\right\|_{s+1} \lesssim s, k \sum_{j=1}^{k}\left\|\widehat{z}_{j}\right\|_{s} \prod_{i \neq j}\left\|\widehat{z}_{i}\right\|_{0}+\left\|z_{\perp}\right\|_{s} \prod_{j=1}^{k}\left\|\widehat{z}_{j}\right\|_{0} .
$$


Proof. By Proposition 3.1 and Corollary 4.1 one has that for any $s \in \mathbb{Z}_{\geq 0}$, the map $\Psi=\Psi_{L} \circ \Psi_{C}: \mathcal{V}_{\delta}^{\prime} \cap h_{r}^{s} \rightarrow H_{r}^{s}$ is real analytic and

$$
\Psi^{*} \Lambda=\left(\Psi_{L} \circ \Psi_{C}\right)^{*} \Lambda=\Psi_{C}^{*} \Psi_{L}^{*} \Lambda \stackrel{(4.1)}{=} \Psi_{C}^{*} \Lambda_{1} \stackrel{\text { Proposition }}{=} 4.1 \Lambda_{0} \stackrel{(4.1)}{=} \Lambda_{M} ，
$$

implying that $\Psi$ is symplectic. Recalling that $\Psi_{L}=F_{\text {nls }}^{-1}+B_{L}$ (see (3.7)) and using that, by Corollary 4.1, $\Psi_{C}=\imath d+B_{C}$, a direct calculation shows that for any $z \in \mathcal{V}_{\delta}^{\prime} \cap h_{r}^{0}$

$$
B(z)=\Psi(z)-F_{\mathrm{nls}}^{-1}(z)=F_{\mathrm{nls}}^{-1}\left(B_{C}(z)\right)+B_{L}\left(\Psi_{C}(z)\right) .
$$

The claimed estimates for $B$ then follow from the estimates of Proposition 3.1 and the ones of Corollary 4.1.

Substituting formula (3.7) for $B_{L}$ one gets

$$
\Psi(z)=F_{\mathrm{nls}}^{-1}(z)+F_{\mathrm{nls}}^{-1}\left(B_{C}(z)\right)+B^{\mathrm{nls}}\left(\Pi_{S} z+\Pi_{S} B_{C}(z)\right)+\mathrm{d}_{\perp} B^{\mathrm{nls}}\left(\Pi_{S} z+\Pi_{S} B_{C}(z)\right)\left[z_{\perp}+\pi_{\perp} B_{C}(z)\right],
$$

where according to Corollary 4.2,

$$
B_{C}(z)=\frac{1}{2} \mathrm{~d}^{2} B_{C}\left(\Pi_{S} z\right)\left[\Pi_{\perp} z, \Pi_{\perp} z\right]+\frac{1}{2} \int_{0}^{1}(1-t)^{2} \mathrm{~d}^{3} B_{C}\left(\Pi_{S} z+t \Pi_{\perp} z\right)\left[\Pi_{\perp} z, \Pi_{\perp} z, \Pi_{\perp} z\right] \mathrm{d} t .
$$

Next, we state and prove the one smoothing property and tame estimates for the map

$$
\mathcal{A}(z):=\mathrm{d} \Psi(z)^{-1}-F_{\text {nls }}, \quad z \in \mathcal{V}_{\delta}^{\prime} \cap h_{r}^{0}
$$

By the chain rule,

$$
\mathrm{d} \Psi(z)^{-1}=\mathrm{d} \Psi_{C}(z)^{-1}\left(\mathrm{~d} \Psi_{L}\left(\Psi_{C}(z)\right)\right)^{-1} .
$$

By Corollary 4.1,

$$
\mathrm{d} \Psi_{C}(z)^{-1}=\mathrm{d} \Psi_{C}^{-1}\left(\Psi_{C}(z)\right)=\mathrm{Id}+\mathrm{d} A_{C}\left(\Psi_{C}(z)\right),
$$

and that by (3.10), $\mathrm{d} \Psi_{L}(z)^{-1}=F_{\mathrm{nls}}+\mathcal{A}_{L}(z)$. Hence (5.6) can be written as

$$
\mathrm{d} \Psi(z)^{-1}=F_{\mathrm{nls}}+\mathcal{A}(z), \quad \mathcal{A}(z):=\mathcal{A}_{L}\left(\Psi_{C}(z)\right)+\mathrm{d} A_{C}\left(\Psi_{C}(z)\right) \mathrm{d} \Psi_{L}\left(\Psi_{C}(z)\right)^{-1} .
$$


1506 T. Kappeler and R. Montalto

Proposition 5.2 (Tame estimates for $\mathcal{A})$. For any $s \in \mathbb{Z}_{\geq 1}$, the map $\mathcal{A}: \mathcal{V}_{\delta}^{\prime} \cap h_{r}^{s} \rightarrow \mathcal{L}\left(H_{c}^{s}, h_{c}^{s+1}\right)$ is real analytic and satisfies the following tame estimates: for any $z \in \mathcal{V}_{\delta}^{\prime} \cap h_{r}^{0}, \widehat{w} \in H_{c}^{0}$,

$$
\|\mathcal{A}(z)[\widehat{W}]\|_{0} \lesssim\|\widehat{W}\|_{0}
$$

and for any $k \geq 1, \widehat{z}_{1}, \ldots, \widehat{z}_{k} \in h_{c}^{0}$

$$
\left\|\mathrm{d}^{k}(\mathcal{A}(z)[\widehat{w}])\left[\widehat{z}_{1}, \ldots, \widehat{z}_{k}\right]\right\|_{0} \lesssim k\|\widehat{w}\|_{0} \prod_{j=1}^{k}\left\|\widehat{z}_{j}\right\|_{0}
$$

Moreover, for any $s \in \mathbb{Z}_{\geq 1}, z \in \mathcal{V}_{\delta}^{\prime} \cap h_{r}^{s}, w \in H_{c}^{s}$,

$$
\|\mathcal{A}(z)[\widehat{W}]\|_{s+1} \lesssim s\left\|z_{\perp}\right\|_{s}\|\widehat{W}\|_{0}+\|\widehat{W}\|_{s}
$$

and for any $k \geq 1, \widehat{z}_{1}, \ldots, \widehat{z}_{k} \in h_{c^{\prime}}^{s}$

$$
\left\|\mathrm{d}^{k}(\mathcal{A}(\boldsymbol{z})[\widehat{W}])\left[\widehat{Z}_{1}, \ldots, \widehat{z}_{k}\right]\right\|_{s+1} \lesssim_{s, k}\left(\left\|z_{\perp}\right\|_{s}\|\widehat{W}\|_{0}+\|\widehat{W}\|_{s}\right) \prod_{j=1}^{k}\left\|\widehat{Z}_{j}\right\|_{0}+\|\widehat{W}\|_{0} \sum_{j=1}^{k}\left\|\widehat{Z}_{j}\right\|_{s} \prod_{i \neq j}\left\|\widehat{z}_{i}\right\|_{0} .
$$

Proof. The claimed estimates for $\mathcal{A}$ follow by Lemma 3.2 and Corollary 4.1 by the chain and product rules.

\subsection{The dNLS Hamiltonian in new coordinates}

In this subsection we prove the expansion of $\mathcal{H}^{\text {nls }} \circ \Psi$, stated in $(C 3)$ of Theorem 1.2. Recall from (1.2) that the Hamiltonian of the dNLS equation is given by

$$
\mathcal{H}^{\mathrm{nls}}(w)=\int_{0}^{1}\left(\partial_{x} u \partial_{X} V+u^{2} v^{2}\right) d x, \quad w=(u, v) \in H_{r}^{1} .
$$

By Theorem 1.1, $H^{\text {nls }}:=\mathcal{H}^{\text {nls }} \circ \Psi^{\text {nls }}$ only depends on the actions. By a slight abuse of notation we write

$$
H^{\mathrm{nls}}=H^{\mathrm{nls}}(I), \quad I=\left(I_{k}\right)_{k \in \mathbb{Z}} \in \ell_{+}^{1,2}, \quad I_{k} \equiv I_{k}(z)=\left|z_{k}\right|^{2} / 2=\left(x_{k}^{2}+y_{k}^{2}\right) / 2 \quad \forall k \in \mathbb{Z}
$$

and denote by $\omega_{k}^{\text {nls }}(I)$ the dNLS frequencies,

$$
\omega_{k}^{\mathrm{nls}}(I):=\partial_{I_{k}} H^{\mathrm{nls}}(I), \quad k \in \mathbb{Z} .
$$


The properties of the frequency map $I \mapsto \omega(I):=\left(\omega_{k}(I)\right)_{k \in \mathbb{Z}}$, needed in the sequel, are summarized in the following.

Proposition 5.3. (dNLS frequencies) The map

$$
\ell_{+}^{1,2} \rightarrow \ell^{\infty},\left(I_{k}\right)_{k \in \mathbb{Z}} \mapsto\left(\omega_{n}^{\mathrm{nls}}(I)-4 \pi^{2} n^{2}\right)_{n \in \mathbb{Z}}
$$

is real analytic and bounded.

Proof. See, for example, Theorem 3.2 in [2].

With the notation introduced above, the $L^{2}$-gradient $\nabla H^{\text {nls }}(z)$ is then given by

$$
\nabla H^{\mathrm{nls}}(z)=\Omega^{\mathrm{nls}}(I)[z], \quad z \in h_{r}^{1}, I \equiv I(z)=\left(I_{n}(z)\right)_{n \in \mathbb{Z},}
$$

where for any $I \in \ell_{+}^{1,2}, \quad \Omega^{\text {nls }}(I): h_{r}^{1} \rightarrow h_{r}^{-1}$ is the diagonal operator

$$
\Omega^{\text {nls }}(I):=\left(\begin{array}{cc}
\operatorname{diag}_{k \in \mathbb{Z}} \omega_{k}^{\text {nls }}(I) & 0 \\
0 & \operatorname{diag}_{k \in \mathbb{Z}} \omega_{k}^{\text {nls }}(I)
\end{array}\right)
$$

Further note that since $H^{\text {nls }}(z)=\mathcal{H}^{\text {nls }}\left(\Psi^{\text {nls }}(z)\right)$ one has by the chain rule

$$
\Omega^{\mathrm{nls}}(I)[z]=\nabla H^{\mathrm{nls}}(z)=\left(d \Psi^{\mathrm{nls}}(z)\right)^{t} \nabla \mathcal{H}^{\mathrm{nls}}\left(\Psi^{\mathrm{nls}}(z)\right), \quad \forall z \in \mathcal{V} \cap h_{r}^{1}
$$

where $\mathcal{V}$ is the neighbourhood of $h_{r}^{0}$ in $h_{c}^{0}$ of Theorem 1.1, $\mathcal{V}=\Phi^{\text {nls }}(\mathcal{W})$. For later use we record that (5.12), evaluated at $z$ with $z=\Pi_{S} z$, reads

$$
\Omega^{\mathrm{nls}}\left(I_{S}, 0\right)\left[\Pi_{S} Z\right]=\left(\mathrm{d} \Psi^{\mathrm{nls}}\left(\Pi_{S} Z\right)\right)^{t} \nabla \mathcal{H}^{\mathrm{nls}}\left(\Psi^{\mathrm{nls}}\left(\Pi_{S} Z\right)\right)
$$

implying that

$$
\Pi_{\perp}\left(\mathrm{d} \Psi^{\mathrm{nls}}\left(\Pi_{S} z\right)\right)^{t} \nabla \mathcal{H}^{\mathrm{nls}}\left(\Psi^{\mathrm{nls}}\left(\Pi_{S} z\right)\right)=0
$$

The equations of motion, associated with the Hamiltonian $H^{\text {nls }}$ are given by

$$
\partial_{t} z=J \Omega^{\mathrm{nls}}(I)[z], \quad J=\left(\begin{array}{cc}
0 & -\mathrm{Id} \\
\mathrm{Id} & 0
\end{array}\right) .
$$


According to the splitting $z=\left(z_{S}, z_{\perp}\right) \in \mathbb{C}^{S} \times \mathbb{C}^{S} \times h_{\perp C}^{0}$, we can decompose the equation (5.14) as

$$
\left\{\begin{array}{l}
\partial_{t} z_{S}=J \Omega_{S}^{\mathrm{nls}}(I)\left[z_{S}\right] \\
\partial_{t} z_{\perp}=J \Omega_{\perp}^{\mathrm{nls}}(I)\left[z_{\perp}\right]
\end{array}\right.
$$

where

$$
\Omega_{S}^{\text {nls }}(I):=\left(\begin{array}{cc}
\operatorname{diag}_{k \in S} \omega_{k}^{\text {nls }}(I) & 0 \\
0 & \operatorname{diag}_{k \in S} \omega_{k}^{\text {nls }}(I)
\end{array}\right), \Omega_{\perp}^{\text {nls }}(I):=\left(\begin{array}{cc}
\operatorname{diag}_{k \in S^{\perp}} \omega_{k}^{\text {nls }}(I) & 0 \\
0 & \operatorname{diag}_{k \in S^{\perp}} \omega_{k}^{\text {nls }}(I)
\end{array}\right) .
$$

Similarly, by a slight abuse of terminology, we identify $I=\left(I_{k}\right)_{k \in \mathbb{Z}}$ with $\left(I_{S}, I_{\perp}\right)$,

$$
I=\left(I_{S}, I_{\perp}\right), \quad I_{S}:=\left(I_{k}\right)_{k \in S}, \quad I_{\perp}:=\left(I_{k}\right)_{k \in S^{\perp}} .
$$

Although the frequencies $\omega_{k}(I)$ are functions of all the action variables $I_{n}, n \in Z$, the system (5.15) decouples since the action variables are invariant in time and depend only on the initial data. Now let us assume that $z(t)=\left(z_{S}(t), 0\right)$ is a solution of (5.15) with initial data $z(0)=\left(z_{S}^{(0)}, 0\right)$ and consider the equation obtained from (5.15) by linearizing it along $\left(z_{S}(t), 0\right)$ with initial data given by $\widehat{z}^{(0)}=\left(0, \widehat{z}_{\perp}^{(0)}\right)$ and $\widehat{z}_{\perp}^{(0)} \in h_{\perp r}^{1}$ and denote by $\widehat{z}(t)$ the corresponding solution which evolves in $h_{r}^{1}$. By a straightforward computation one verifies that the differential of $\Omega^{\text {nls }}(I)$ at $\left(z_{S}^{(0)}, 0\right)$ in direction $\left(0, \widehat{z}_{\perp}^{(0)}\right)$ vanishes, implying that $\widehat{z}(t)=\left(0, \widehat{z}_{\perp}(t)\right)$ where $\widehat{z}_{\perp}(t)$ is the solution of

$$
\partial_{t} \widehat{z}_{\perp}(t)=J \Omega_{\perp}\left(I_{S}, 0\right)\left[\widehat{z}_{\perp}(t)\right], \quad \widehat{z}_{\perp}(0)=\widehat{z}_{\perp}^{(0)} .
$$

Since by Theorem 1.1, $\Psi^{\text {nls }}: h_{r}^{1} \rightarrow H_{r}^{1}$ is symplectic it follows that

$$
\widehat{W}(t):=\mathrm{d} \Psi^{\mathrm{nls}}\left(z_{S}(t), 0\right)\left[\left(0, \widehat{z}_{\perp}(t)\right)\right]=\mathrm{d}_{\perp} \Psi^{\mathrm{nls}}\left(z_{S}(t), 0\right)\left[\widehat{z}_{\perp}(t)\right]
$$

is a solution of the equation obtained by linearizing the dNLS equation along $\Psi^{\mathrm{nls}}\left(z_{S}(t), 0\right)$. More precisely,

$$
\partial_{t} \widehat{W}(t)=\mathrm{i} \rrbracket \mathrm{d} \nabla \mathcal{H}^{\mathrm{nls}}\left(\Psi^{\mathrm{nls}}\left(z_{S}(t), 0\right)\right)[\widehat{W}(t)], \quad \widehat{W}(0)=\mathrm{d} \Psi^{\mathrm{nls}}\left(z_{S}^{(0)}, 0\right)\left[\left(0, \widehat{z}_{\perp}^{(0)}\right)\right]
$$

On the other hand, by differentiating formula (5.19) with respect to $t$, one gets

$$
\begin{aligned}
\partial_{t} \widehat{W}(t) & =\mathrm{d}_{\perp} \Psi^{\mathrm{nls}}\left(z_{S}(t), 0\right)\left[\partial_{t} \widehat{z}_{\perp}(t)\right]+\mathrm{d}_{S}\left(\mathrm{~d}_{\perp} \Psi^{\mathrm{nls}}\left(z_{S}(t), 0\right)\left[\widehat{z}_{\perp}(t)\right]\right)\left[\partial_{t} z_{S}(t)\right] \\
& =\mathrm{d}_{\perp} \Psi^{\mathrm{nls}}\left(z_{S}(t), 0\right)\left[J \Omega_{\perp}^{\mathrm{nls}}\left(I_{S}, 0\right) \widehat{z}_{\perp}(t)\right]+\mathrm{d}_{S}\left(\mathrm{~d}_{\perp} \Psi^{\mathrm{nls}}\left(z_{S}(t), 0\right)\left[\widehat{z}_{\perp}(t)\right]\right)\left[J \Omega_{S}^{\mathrm{nls}}\left(I_{S}, 0\right) z_{S}(t)\right]
\end{aligned}
$$


Comparing (5.20) and (5.21) one gets

$$
\begin{aligned}
\mathrm{i} \rrbracket d \nabla \mathcal{H}^{\mathrm{nls}}\left(\Psi^{\mathrm{nls}}\left(z_{S}(t), 0\right)\right)\left[\mathrm{d}_{\perp} \Psi^{\mathrm{nls}}\left(z_{S}(t), 0\right) \widehat{z}_{\perp}\right]=\mathrm{d}_{\perp} \Psi^{\mathrm{nls}}\left(z_{S}(t), 0\right)\left[J \Omega_{\perp}^{\mathrm{nls}}\left(I_{S}, 0\right) \widehat{z}_{\perp}(t)\right] \\
+\mathrm{d}_{S}\left(\mathrm{~d}_{\perp} \Psi^{\mathrm{nls}}\left(z_{S}(t), 0\right)\left[\widehat{z}_{\perp}(t)\right]\right)\left[J \Omega_{S}^{\mathrm{nls}}\left(I_{S}, 0\right) z_{S}(t)\right] .
\end{aligned}
$$

The latter identity implies that for any $z_{S} \in \mathbb{R}^{S} \times \mathbb{R}^{S}, \widehat{z}_{\perp} \in h_{\perp r}^{1}$

$$
\begin{gathered}
\mathrm{i} \rrbracket \mathrm{d} \nabla \mathcal{H}^{\mathrm{nls}}\left(\Psi^{\mathrm{nls}}\left(z_{S}, 0\right)\right)\left[\mathrm{d} \Psi^{\mathrm{nls}}\left(z_{S}, 0\right)\left[\left(0, \widehat{z}_{\perp}\right)\right]\right]=\mathrm{d} \Psi^{\mathrm{nls}}\left(z_{S}, 0\right) J \Omega^{\mathrm{nls}}\left(I_{S}, 0\right)\left[\left(0, \widehat{z}_{\perp}\right)\right] \\
+\mathrm{d}_{S}\left(\mathrm{~d}_{\perp} \Psi^{\mathrm{nls}}\left(z_{S}, 0\right)\left[\widehat{z}_{\perp}\right]\right)\left[J \Omega_{S}^{\mathrm{nls}}\left(I_{S}, 0\right) z_{S}\right] .
\end{gathered}
$$

Solving for $J \Omega^{\text {nls }}\left(I_{S}, 0\right)\left[\left(0, \widehat{z}_{\perp}\right)\right]$, one gets

$$
\begin{aligned}
J \Omega^{\mathrm{nls}}\left(I_{S}, 0\right)\left[\left(0, \widehat{z}_{\perp}\right)\right]=\left(\mathrm{d} \Psi^{\mathrm{nls}}\left(z_{S}, 0\right)\right)^{-1} \mathrm{i} \rrbracket \mathrm{d} \nabla \mathcal{H}^{\mathrm{nls}}\left(\Psi^{\mathrm{nls}}\left(z_{S}, 0\right)\right)\left[\mathrm{d} \Psi^{\mathrm{nls}}\left(z_{S}, 0\right)\left(0, \widehat{z}_{\perp}\right)\right] \\
-\left(\mathrm{d} \Psi^{\mathrm{nls}}\left(z_{S}, 0\right)\right)^{-1} \mathrm{~d}_{S}\left(\mathrm{~d}_{\perp} \Psi^{\mathrm{nls}}\left(z_{S}, 0\right)\left[\widehat{z}_{\perp}\right]\right)\left[J \Omega_{S}^{\mathrm{nls}}\left(I_{S}, 0\right) z_{S}\right] .
\end{aligned}
$$

Since $\Psi^{\text {nls }}$ is symplectic, one has

$$
\left(\mathrm{d} \Psi^{\mathrm{nls}}\left(z_{S}, 0\right)\right)^{-1} \mathrm{i} \mathbb{J}=J\left(\mathrm{~d} \Psi^{\mathrm{nls}}\left(z_{S}, 0\right)\right)^{t}, \quad\left(\mathrm{~d} \Psi^{\mathrm{nls}}\left(z_{S}, 0\right)\right)^{-1}=J\left(\mathrm{~d} \Psi^{\mathrm{nls}}\left(z_{S}, 0\right)\right)^{t} \mathbf{i} \mathbb{J}
$$

and hence (5.24) reads

$$
\Omega^{\mathrm{nls}}\left(I_{S}, 0\right)\left[\left(0, \widehat{z}_{\perp}\right)\right]=\left(\mathrm{d} \Psi^{\mathrm{nls}}\left(z_{S}, 0\right)\right)^{t} \mathrm{~d} \nabla \mathcal{H}^{\mathrm{nls}}\left(\Psi^{\mathrm{nls}}\left(z_{S}, 0\right)\right) \mathrm{d} \Psi^{\mathrm{nls}}\left(z_{S}, 0\right)\left[\left(0, \widehat{z}_{\perp}\right)\right]-\mathcal{R}^{(1)}\left(z_{S}\right)\left[\widehat{z}_{\perp}\right],
$$

where $\mathcal{R}^{(1)}\left(z_{S}\right): h_{\perp c}^{0} \rightarrow h_{c}^{0}$ is the bounded linear operator, defined by

$$
\mathcal{R}^{(1)}\left(z_{S}\right)\left[\widehat{z}_{\perp}\right]:=\mathrm{d} \Psi^{\mathrm{nls}}\left(z_{S}, 0\right)^{t} \mathrm{i} \rrbracket \mathrm{d}_{S}\left(\mathrm{~d}_{\perp} \Psi^{\mathrm{nls}}\left(z_{S}, 0\right)\left[\widehat{z}_{\perp}\right]\right)\left[J \Omega_{S}^{\mathrm{nls}}\left(I_{S}, 0\right) z_{S}\right]
$$

For later use we record the following estimates for $\mathcal{R}^{(1)}\left(z_{S}\right)$.

Lemma 5.1. The map $\mathcal{V}_{S} \cap\left(\mathbb{R}^{S} \times \mathbb{R}^{S}\right) \rightarrow \mathcal{L}\left(h_{\perp c}^{0}, h_{c}^{0}\right), z_{S} \mapsto \mathcal{R}^{(1)}\left(z_{S}\right)$ is real analytic and bounded. Moreover it is one smoothing, meaning that for any $s \in \mathbb{Z}_{\geq 1}, \mathcal{V}_{S} \cap\left(\mathbb{R}^{S} \times \mathbb{R}^{S}\right) \rightarrow$ $\mathcal{L}\left(h_{\perp c}^{s}, h_{c}^{s+1}\right), z_{S} \mapsto \mathcal{R}^{(1)}\left(z_{S}\right)$ is real analytic. Furthermore, for any $s \in \mathbb{Z}_{\geq 1}, \alpha, \beta \in \mathbb{Z}_{\geq 0}^{S}$, $z_{S} \in \mathcal{V}_{S} \cap\left(\mathbb{R}^{S} \times \mathbb{R}^{S}\right)$,

$$
\left\|\partial_{S}^{\alpha, \beta} \mathcal{R}^{(1)}\left(z_{S}\right)\right\|_{\mathcal{L}\left(h_{\perp C}^{0}, h_{C}^{0}\right)} \lesssim_{\alpha, \beta} \quad 1, \quad\left\|\partial_{S}^{\alpha, \beta} \mathcal{R}^{(1)}\left(z_{S}\right)\right\|_{\mathcal{L}\left(h_{\perp C}^{s}, h_{C}^{s+1}\right)} \lesssim_{s, \alpha, \beta} \quad 1
$$

Proof. By Theorem 1.1, $\Psi^{\mathrm{nls}}=F_{\mathrm{nls}}^{-1}+B^{\mathrm{nls}}$ and hence $\mathrm{d}_{S}\left(\mathrm{~d}_{\perp} \Psi^{\mathrm{nls}}\left(z_{S}, 0\right)\left[\widehat{z}_{\perp}\right]\right)=$ $\mathrm{d}_{S}\left(\mathrm{~d}_{\perp} B^{\mathrm{nls}}\left(z_{S}, 0\right)\left[\widehat{z}_{\perp}\right]\right)$. The claimed statements then follow from Theorem 1.1. 
We also need to record some properties of the operator $\Omega_{\perp}^{\text {nls }}(I)$ for $I=\left(I_{S}, 0\right)$. Write

$$
\Omega_{\perp}^{\mathrm{nls}}\left(I_{S}, 0\right)=D_{\perp}^{2}+\Omega_{\perp}^{(0)}\left(I_{S}, 0\right)
$$

where

$$
D_{\perp}:=\left(\begin{array}{cc}
\operatorname{diag}_{n \in S^{\perp}}(2 \pi n) & 0 \\
0 & \operatorname{diag}_{n \in S^{\perp}}(2 \pi n)
\end{array}\right)
$$

and

$$
\Omega_{\perp}^{(0)}\left(I_{S}, 0\right):=\left(\begin{array}{cc}
\operatorname{diag}_{n \in S^{\perp}}\left(\omega_{n}^{\mathrm{nls}}\left(I_{S}, 0\right)-4 \pi^{2} n^{2}\right) & 0 \\
0 & \operatorname{diag}_{n \in S^{\perp}}\left(\omega_{n}^{\mathrm{nls}}\left(I_{S}, 0\right)-4 \pi^{2} n^{2}\right)
\end{array}\right)
$$

Lemma 5.2. For any $s \in \mathbb{Z}_{\geq 0}$, the $\operatorname{map} \mathcal{V}_{S} \cap\left(\mathbb{R}^{S} \times \mathbb{R}^{S}\right) \rightarrow \mathcal{L}\left(h_{\perp c}^{s}, h_{\perp c}^{s}\right), z_{S} \mapsto \Omega_{\perp}^{(0)}\left(I_{S}\left(z_{S}\right), 0\right)$ is real analytic and bounded.

Proof. The lemma is a straightforward application of Proposition 5.3, since for any $\alpha, \beta \in \mathbb{Z}_{\geq 0}^{S}$

$$
\sup _{n \in S^{\perp}}\left|\partial_{S}^{\alpha, \beta}\left(\omega_{n}^{\text {nls }}\left(I_{S}, 0\right)-4 \pi^{2} n^{2}\right)\right| \lesssim_{\alpha, \beta} \quad 1
$$

and

$$
\left\|\partial_{S}^{\alpha, \beta} \Omega_{\perp}^{(0)}\left(I_{S}, 0\right)\right\|_{\mathcal{L}\left(h_{\perp C^{\prime}}^{s}, h_{\perp C}^{s}\right)} \lesssim \sup _{n \in S^{\perp}}\left|\partial_{S}^{\alpha, \beta}\left(\omega_{n}^{\mathrm{nls}}\left(I_{S}, 0\right)-4 \pi^{2} n^{2}\right)\right| \lesssim \alpha, \beta \quad 1
$$

uniformly on $\mathcal{V}_{S} \cap\left(\mathbb{R}^{S} \times \mathbb{R}^{S}\right)$.

After this preliminary discussion, we can now study the transformed Hamiltonian $\mathcal{H}^{\text {nls }}$ 。 $\Psi$ where $\Psi=\Psi_{L} \circ \Psi_{C}$ is the symplectic transformation introduced in Section 5.1. We split the analysis into two parts. First we expand $\mathcal{H}^{(1)}:=\mathcal{H}^{\text {nls }} \circ \Psi_{L}$ and then we analyse $\mathcal{H}^{(2)}=\mathcal{H}^{(1)} \circ \Psi_{C}$.

\subsubsection{Expansion of $\mathcal{H}^{\text {nls }} \circ \Psi_{L}$}

To expand $\mathcal{H}^{\text {nls }} \circ \Psi_{L}$, it is useful to write $\mathcal{H}^{\text {nls }}$ in the form

$$
\mathcal{H}^{\text {nls }}(w)=\mathcal{H}_{2}^{\text {nls }}(w)+\mathcal{H}_{4}^{\text {nls }}(w),
$$


where

$$
\mathcal{H}_{2}^{\mathrm{nls}}(w):=\frac{1}{2}\left\langle\mathcal{D}_{2} w, w\right\rangle_{r^{\prime}} \quad \mathcal{H}_{4}^{\mathrm{nls}}(w):=\int_{\mathbb{T}} u^{2} v^{2} \mathrm{~d} x
$$

and the operator $\mathcal{D}_{2}$ is defined as

$$
\mathcal{D}_{2}:=\left(\begin{array}{cc}
0 & -\partial_{X X} \\
-\partial_{X X} & 0
\end{array}\right)
$$

Note that $\mathcal{D}_{2}=\mathcal{D}_{2}^{t}$. The Hamiltonian equations associated with (5.30) can be written as

$$
\partial_{t} w=\mathrm{i} \mathbb{J} \nabla \mathcal{H}^{\mathrm{nls}}(w), \quad \mathbb{J}=\left(\begin{array}{cc}
0 & -\mathrm{Id} \\
\mathrm{Id} & 0
\end{array}\right), \quad \nabla \mathcal{H}^{\mathrm{nls}}=\left(\nabla_{u} \mathcal{H}^{\mathrm{nls}}, \nabla_{\mathrm{v}} \mathcal{H}^{\mathrm{nls}}\right),
$$

where

$$
\nabla \mathcal{H}^{\mathrm{nls}}(w)=\mathcal{D}_{2} w+\nabla \mathcal{H}_{4}^{\mathrm{nls}}(w), \quad \mathrm{d} \nabla \mathcal{H}^{\mathrm{nls}}(w)=\mathcal{D}_{2}+\mathrm{d} \nabla \mathcal{H}_{4}^{\mathrm{nls}}(w)
$$

The Taylor expansion of $\mathcal{H}^{\text {nls }}$ around $\Psi^{\text {nls }}\left(\Pi_{S} Z\right)$ up to order three reads

$$
\begin{aligned}
\mathcal{H}^{\mathrm{nls}}\left(\Psi^{\mathrm{nls}}\left(\Pi_{S} z\right)+w\right)= & \mathcal{H}^{\mathrm{nls}}\left(\Psi^{\mathrm{nls}}\left(\Pi_{S} z\right)\right)+\left\langle\nabla \mathcal{H}^{\mathrm{nls}}\left(\Psi^{\mathrm{nls}}\left(\Pi_{S} z\right)\right), w\right\rangle_{r} \\
& +\frac{1}{2}\left\langle\mathrm{~d} \nabla \mathcal{H}^{\mathrm{nls}}\left(\Psi^{\mathrm{nls}}\left(\Pi_{S} z\right)\right)[w], w\right\rangle_{r}+\mathcal{T}_{3}^{(1)}\left(z_{S}, w\right),
\end{aligned}
$$

where $\mathcal{T}_{3}^{(1)}\left(z_{S}, w\right)$ is the Taylor remainder term of order three, given by

$$
\begin{aligned}
& \mathcal{T}_{3}^{(1)}\left(z_{S}, w\right):=\frac{1}{2} \int_{0}^{1}(1-t)^{2} \mathrm{~d}^{3} \mathcal{H}^{\mathrm{nls}}\left(\Psi^{\mathrm{nls}}\left(\Pi_{S} z\right)+t w\right)[w, w, w] \mathrm{d} t \\
& \stackrel{(5.30),(5.31)}{=} \frac{1}{2} \int_{0}^{1}(1-t)^{2} \mathrm{~d}^{3} \mathcal{H}_{4}^{\mathrm{nls}}\left(\Psi^{\mathrm{nls}}\left(\Pi_{S} z\right)+t w\right)[w, w, w] \mathrm{d} t
\end{aligned}
$$

For later use we record that the third derivative of $\mathcal{H}_{4}^{\text {nls }}$ at $w_{0}=\left(u_{0}, v_{0}\right) \in H_{r}^{1}$ in direction $w=(u, v)$ in $H_{r}^{1}$ can be computed as

$$
\mathrm{d}^{3} \mathcal{H}_{4}^{\mathrm{nls}}\left(w_{0}\right)[w, w, w]=12 \int_{0}^{1}\left(u_{0} u v^{2}+u^{2} v_{0} v\right) \mathrm{d} x
$$

Substituting for $w$ the function $\mathrm{d}_{\perp} \Psi^{\mathrm{nls}}\left(\Pi_{S} z\right)\left[z_{\perp}\right]\left(=\mathrm{d} \Psi^{\mathrm{nls}}\left(\Pi_{S} z\right)\left[\Pi_{\perp} z\right]\right)$ and taking into account that by (3.3), $\Psi_{L}(z)=\Psi^{\mathrm{nls}}\left(\Pi_{S} z\right)+\mathrm{d}_{\perp} \Psi^{\mathrm{nls}}\left(\Pi_{S} z\right)\left[z_{\perp}\right]$ yields

$$
\mathcal{H}^{(1)}(z)=\mathcal{H}^{\mathrm{nls}}\left(\Psi_{L}(z)\right)=\mathcal{H}^{\mathrm{nls}}\left(\Psi^{\mathrm{nls}}\left(\Pi_{S} z\right)\right)+\left\langle\nabla \mathcal{H}^{\mathrm{nls}}\left(\Psi^{\mathrm{nls}}\left(\Pi_{S} z\right)\right), \mathrm{d} \Psi^{\mathrm{nls}}\left(\Pi_{S} z\right)\left[\Pi_{\perp} z\right]\right\rangle_{r}
$$


1512 T. Kappeler and R. Montalto

$$
\begin{aligned}
+ & \frac{1}{2}\left\langle\mathrm{~d} \nabla \mathcal{H}^{\mathrm{nls}}\left(\Psi^{\mathrm{nls}}\left(\Pi_{S} z\right)\right)\left[\mathrm{d} \Psi^{\mathrm{nls}}\left(\Pi_{S} z\right)\left[\Pi_{\perp} z\right]\right], \mathrm{d} \Psi^{\mathrm{nls}}\left(\Pi_{S} z\right)\left[\Pi_{\perp} z\right]\right\rangle_{r} \\
& +\mathcal{T}_{3}^{(1)}\left(z_{S}, \mathrm{~d} \Psi^{\mathrm{nls}}\left(\Pi_{S} z\right)\left[\Pi_{\perp} z\right]\right)
\end{aligned}
$$

Writing the right-hand side of the latter identity in a more convenient form one gets

$$
\begin{aligned}
\mathcal{H}^{(1)}(z)= & \mathcal{H}^{\mathrm{nls}}\left(\Psi^{\mathrm{nls}}\left(\Pi_{S} z\right)\right)+\left(\Pi_{\perp}\left(\mathrm{d} \Psi^{\mathrm{nls}}\left(\Pi_{S} z\right)\right)^{t} \nabla \mathcal{H}^{\mathrm{nls}}\left(\Psi^{\mathrm{nls}}\left(\Pi_{S} z\right)\right), \Pi_{\perp} z\right)_{r} \\
+ & \frac{1}{2}\left(\Pi_{\perp}\left(\mathrm{d} \Psi^{\mathrm{nls}}\left(\Pi_{S} z\right)\right)^{t} \mathrm{~d} \nabla \mathcal{H}^{\mathrm{nls}}\left(\Psi^{\mathrm{nls}}\left(\Pi_{S} z\right)\right) \mathrm{d} \Psi^{\mathrm{nls}}\left(\Pi_{S} z\right)\left[\Pi_{\perp} z\right], \Pi_{\perp} z\right)_{r} \\
& +\mathcal{T}_{3}^{(1)}\left(z_{S}, \mathrm{~d} \Psi^{\mathrm{nls}}\left(\Pi_{S} z\right)\left[\Pi_{\perp} z\right]\right) .
\end{aligned}
$$

Recall that $H^{\text {nls }}=\mathcal{H}^{\text {nls }} \circ \Psi^{\text {nls }}$. Hence by Theorem 1.1 one gets

$$
\mathcal{H}^{\mathrm{nls}}\left(\Psi^{\mathrm{nls}}\left(\Pi_{S} Z\right)\right)=H^{\mathrm{nls}}\left(I_{S}, 0\right)
$$

Furthermore by (5.13),

$$
\Pi_{\perp}\left(\mathrm{d} \Psi^{\mathrm{nls}}\left(\Pi_{S} Z\right)\right)^{t} \nabla \mathcal{H}^{\mathrm{nls}}\left(\Psi^{\mathrm{nls}}\left(\Pi_{S} Z\right)\right)=0
$$

Next, the term in (5.37), which is quadratic in $z_{\perp}$, can be written as

$$
\begin{aligned}
& \frac{1}{2}\left(\Pi_{\perp}\left(\mathrm{d} \Psi^{\mathrm{nls}}\left(\Pi_{S} z\right)\right)^{t} \mathrm{~d} \nabla \mathcal{H}^{\mathrm{nls}}\left(\Psi^{\mathrm{nls}}\left(\Pi_{S} z\right)\right) \mathrm{d} \Psi^{\mathrm{nls}}\left(\Pi_{S} z\right)\left[\Pi_{\perp} z\right], \Pi_{\perp} z\right)_{r} \\
& \stackrel{(5.25)}{=} \frac{1}{2}\left(\Omega^{\mathrm{nls}}\left(I_{S}, 0\right)\left[\Pi_{\perp} z\right], \Pi_{\perp} z\right)_{r}+\frac{1}{2}\left(\mathcal{R}^{(1)}\left(z_{S}\right)\left[z_{\perp}\right], z_{\perp}\right)_{r} .
\end{aligned}
$$

Substituting (5.38-5.40) into (5.37) then yields

$$
\mathcal{H}^{(1)}(z)=H^{\mathrm{nls}}\left(I_{S}, 0\right)+\frac{1}{2}\left(\Omega_{\perp}^{\mathrm{nls}}\left(I_{S}, 0\right)\left[z_{\perp}\right], z_{\perp}\right)_{r}+\mathcal{P}_{2}^{(1)}(z)+\mathcal{P}_{3}^{(1)}(z)
$$

where

$$
\mathcal{P}_{2}^{(1)}(z):=\frac{1}{2}\left(\mathcal{R}^{(1)}\left(z_{S}\right)\left[z_{\perp}\right], z_{\perp}\right)_{r^{\prime}} \quad \mathcal{P}_{3}^{(1)}(z):=\mathcal{T}_{3}^{(1)}\left(z_{S}, \mathrm{~d} \Psi^{\mathrm{nls}}\left(\Pi_{S} z\right)\left[\Pi_{\perp} z\right]\right) .
$$

Lemma 5.3. (i) For any $s \in \mathbb{Z}_{\geq 0}, \mathcal{P}_{2}^{(1)}: \mathcal{V} \cap h_{r}^{s} \rightarrow \mathbb{R}$ is real analytic and the following estimates hold: for any $s \in \mathbb{Z}_{\geq 0}, z \in \mathcal{V} \cap h_{r}^{s}$,

$$
\left\|\nabla \mathcal{P}_{2}^{(1)}(z)\right\|_{s} \lesssim_{s}\left\|z_{\perp}\right\|_{s}
$$


and for any $k \in \mathbb{Z}_{\geq 1}, \widehat{z}_{1}, \ldots, \widehat{z}_{k} \in h_{c^{\prime}}^{s}$

$$
\left\|\mathrm{d}^{k} \nabla \mathcal{P}_{2}^{(1)}(z)\left[\widehat{z}_{1}, \ldots, \widehat{,}_{k}\right]\right\|_{s} \lesssim_{s, k} \sum_{j=1}^{k}\left\|\widehat{z}_{j}\right\|_{s} \prod_{i \neq j}\left\|\widehat{z}_{i}\right\|_{0}+\left\|z_{\perp}\right\|_{s} \prod_{j=1}^{k}\left\|\widehat{z}_{j}\right\|_{0} .
$$

(ii) For any $s \in \mathbb{Z}_{\geq 0}, \mathcal{P}_{3}^{(1)}: \mathcal{V} \cap h_{r}^{s} \rightarrow \mathbb{R}$ is real analytic and the following estimates hold: for any $s \in \mathbb{Z}_{\geq 0}, z \in \mathcal{V} \cap h_{r}^{s}, \widehat{z} \in h_{c}^{s}$,

$$
\left\|\nabla \mathcal{P}_{3}^{(1)}(z)\right\|_{s} \lesssim_{s}\left\|z_{\perp}\right\|_{s}\left\|z_{\perp}\right\|_{0}, \quad\left\|\mathrm{~d} \nabla \mathcal{P}_{3}^{(1)}(z)[\widehat{Z}]\right\|_{s} \lesssim_{s}\left\|z_{\perp}\right\|_{s}\|\widehat{Z}\|_{0}+\left\|z_{\perp}\right\|_{0}\|\widehat{Z}\|_{s}
$$

and for any $k \in \mathbb{Z}_{\geq 2}, \widehat{z}_{1}, \ldots, \widehat{z}_{k} \in h_{c^{\prime}}^{s}$

$$
\left\|\mathrm{d}^{k} \nabla \mathcal{P}_{3}^{(1)}(z)\left[\widehat{z}_{1}, \ldots, \widehat{z}_{k}\right]\right\|_{s} \grave{s}_{s, k} \sum_{j=1}^{k}\left\|\widehat{z}_{j}\right\|_{s} \prod_{i \neq j}\left\|\widehat{z}_{i}\right\|_{0}+\left\|z_{\perp}\right\|_{s} \prod_{j=1}^{k}\left\|\widehat{z}_{j}\right\|_{0} .
$$

Proof. Item (i) follows from Lemma 5.1 and item (ii) from (5.42), (5.35), (5.36), and Theorem 1.1.

\subsubsection{Expansion of $\mathcal{H}^{(2)}:=\mathcal{H}^{(1)} \circ \Psi_{C}$}

To study the expansion of the composition $\mathcal{H}^{(2)}=\mathcal{H}^{(1)} \circ \Psi_{C}$ of the Hamiltonian $\mathcal{H}^{(1)}$ with the symplectic corrector $\Psi_{C}$, constructed in Section 4, we separately expand the compositions of the terms on the right-hand side of the identity (5.41) with $\Psi_{C}$. In addition to the projectors $\Pi_{S}, \Pi_{\perp}$, defined in (3.1), (3.2), we also introduce the following versions of them,

$$
\begin{gathered}
\pi_{S}: \mathbb{C}^{S} \times \mathbb{C}^{S} \times h_{\perp c}^{0} \rightarrow \mathbb{C}^{S} \times \mathbb{C}^{S}, \quad z=\left(z_{S}, z_{\perp}\right) \rightarrow z_{S}, \\
\pi_{\perp}: \mathbb{C}^{S} \times \mathbb{C}^{S} \times h_{\perp c}^{0} \rightarrow h_{\perp c^{\prime}}^{0} \quad z=\left(z_{S}, z_{\perp}\right) \rightarrow z_{\perp} .
\end{gathered}
$$

5.2.2.1 Term $H^{\text {nls }}\left(I_{S}, 0\right)$. It is convenient to define

$$
h^{\mathrm{nls}}\left(z_{S}\right):=H^{\mathrm{nls}}\left(I_{S}, 0\right) \text {, }
$$

where we recall that by (5.8), (5.17)

$$
I_{S}=I_{S}\left(z_{S}\right)=\left(\frac{1}{2}\left(x_{j}^{2}+y_{j}^{2}\right)\right)_{j \in S}, \quad z_{S}=\left(\left(x_{j}\right)_{j \in S},\left(y_{j}\right)_{j \in S}\right) \in \mathbb{R}^{S} \times \mathbb{R}^{S} .
$$

By Corollaries 4.1, 4.2 $\Psi_{C}(z)$, defined for $z \in \mathcal{V}_{\delta}^{\prime} \cap h_{r}^{0}$, is of the form $\Psi_{C}(z)=z+B_{C}(z)=$ $z+B_{2}^{C}(z)+B_{3}^{C}(z)$. Hence the Taylor expansion of $h^{\text {nls }}\left(\pi_{S} \Psi_{C}(z)\right)$ around $z_{S}$ reads

$$
h^{\mathrm{nls}}\left(\pi_{S} \Psi_{C}(z)\right)=h^{\mathrm{nls}}\left(z_{S}\right)+\nabla_{S} h^{\mathrm{nls}}\left(z_{S}\right) \cdot \pi_{S} B_{2}^{C}(z)+\mathcal{P}_{3}^{(2 a)}(z),
$$


1514 T. Kappeler and R. Montalto

where $\mathcal{P}_{3}^{(2 a)}(z)$ is the Taylor remainder term of order three, given by

$$
\mathcal{P}_{3}^{(2 a)}(z):=\nabla_{S} h^{\mathrm{nls}}\left(z_{S}\right) \cdot \pi_{S} B_{3}^{C}(z)+\int_{0}^{1}(1-t) \mathrm{d}_{S} \nabla_{S} h^{\mathrm{nls}}\left(z_{S}+t \pi_{S} B_{C}(z)\right)\left[\pi_{S} B_{C}(z)\right] \cdot \pi_{S} B_{C}(z) \mathrm{d} t .
$$

In the next lemma we provide estimates for the Hamiltonian $\mathcal{P}_{3}^{(2 a)}(z)$.

Lemma 5.4. For any $s \in \mathbb{Z}_{\geq 0}, \mathcal{P}_{3}^{(2 a)} \circ \Psi_{C}: \mathcal{V}_{\delta}^{\prime} \cap h_{r}^{s} \rightarrow \mathbb{R}$ is real analytic. Furthermore, $\nabla \mathcal{P}_{3}^{(2 a)}$ satisfies the following tame estimates: for any $s \in \mathbb{Z}_{\geq 0}, z \in \mathcal{V}_{\delta}^{\prime} \cap h_{r}^{s}, \widehat{z} \in h_{c^{\prime}}^{s}$

$$
\left\|\nabla \mathcal{P}_{3}^{(2 a)}(z)\right\|_{s} \lesssim s\left\|z_{\perp}\right\|_{s}\left\|z_{\perp}\right\|_{0}, \quad\left\|\mathrm{~d} \nabla \mathcal{P}_{3}^{(2 a)}(z)[\widehat{z}]\right\|_{s} \lesssim_{s}\left\|z_{\perp}\right\|_{s}\|\widehat{Z}\|_{0}+\left\|z_{\perp}\right\|_{0}\|\widehat{Z}\|_{s}
$$

and for any $k \in \mathbb{Z}_{\geq 2}, \widehat{z}_{1}, \ldots, \widehat{z}_{k} \in h_{c}^{s}$

$$
\left\|\mathrm{d}^{k} \nabla \mathcal{P}_{3}^{(2 a)}(z)\left[\widehat{z}_{1}, \ldots, \widehat{z}_{k}\right]\right\|_{s} \lesssim_{s, k} \sum_{j=1}^{k}\left\|\widehat{z}_{j}\right\|_{s} \prod_{i \neq j}\left\|\widehat{z}_{i}\right\|_{0}+\left\|z_{\perp}\right\|_{s} \prod_{j=1}^{k}\left\|\widehat{z}_{j}\right\|_{0} .
$$

Proof. The lemma follows by differentiating $\mathcal{P}_{3}^{(2 a)}$ and applying the estimates of Corollaries 4.1 and 4.2 .

5.2.2.2 Term $\mathcal{H}_{\Omega}(z):=\frac{1}{2}\left(\Omega_{\perp}^{\text {nls }}\left(I_{S}, 0\right) z_{\perp}, z_{\perp}\right)_{r}$. To begin with let us point out that the expansion of the composition of the term $\mathcal{H}_{\Omega}(z)$ with the transformation $\Psi_{C}$ needs special care. To explain this in more detail, write $2 \mathcal{H}_{\Omega}(z)$ in the form

$$
\left(\Omega_{\perp}^{\mathrm{nls}}\left(I_{S}, 0\right) z_{\perp}, z_{\perp}\right)_{r}=\left(D_{\perp}^{2} z_{\perp}, z_{\perp}\right)_{r}+\left(\Omega_{\perp}^{(0)}\left(I_{S}, 0\right) z_{\perp}, z_{\perp}\right)_{r^{\prime}}
$$

where $D_{\perp}$ is the diagonal operator defined in (5.28). When composed with $\Psi_{C}=\iota d+B_{C}$, the term $\left(D_{\perp}^{2} z_{\perp}, z_{\perp}\right)_{r}$ becomes

$$
\begin{gathered}
\left(D_{\perp}^{2}\left[z_{\perp}+\pi_{\perp} B_{C}(z)\right], z_{\perp}+\pi_{\perp} B_{C}(z)\right)_{r}=\left(D_{\perp}^{2}\left[z_{\perp}\right], z_{\perp}\right)_{r}+\left(D_{\perp}^{2}\left[z_{\perp}\right], \pi_{\perp} B_{C}(z)\right)_{r} \\
+\left(D_{\perp}^{2}\left[\pi_{\perp} B_{C}(z)\right], z_{\perp}\right)_{r}+\left(D_{\perp}^{2}\left[\pi_{\perp} B_{C}(z)\right], \pi_{\perp} B_{C}(z)\right)_{r^{\prime}}
\end{gathered}
$$

where $\pi_{\perp}$ is defined in (5.44). By (4.29) and (4.30), it then follows that the difference

$$
\frac{1}{2}\left(D_{\perp}^{2}\left[z_{\perp}+\pi_{\perp} B_{C}(z)\right], z_{\perp}+\pi_{\perp} B_{C}(z)\right)_{r}-\frac{1}{2}\left(D_{\perp}^{2}\left[z_{\perp}\right], z_{\perp}\right)_{r}
$$

belongs to the error term $\mathcal{P}_{3}(z)$ in Theorem 1.2. Since $B_{C}$ is only one smoothing, the two terms

$$
\left(D_{\perp}^{2}\left[z_{\perp}\right], \pi_{\perp} B_{C}(z)\right)_{r^{\prime}} \quad\left(D_{\perp}^{2}\left[\pi_{\perp} B_{C}(z)\right], z_{\perp}\right)_{r}
$$

could prevent that $\mathcal{P}_{3}$ satisfies the estimates (1.7), stated in Theorem 1.2. 
To proceed, recall that $\Psi_{C}=\Psi_{X}^{0,1}$ where $\Psi_{X}^{\tau_{0}, \tau}$ is the flow map, defined in (4.16). We have

$$
\mathcal{H}_{\Omega}\left(\Psi_{C}(z)\right)=\mathcal{H}_{\Omega}(z)+\mathcal{P}_{3}^{(2 b)}(z), \quad \mathcal{P}_{3}^{(2 b)}(z):=\mathcal{H}_{\Omega}\left(\Psi_{C}(z)\right)-\mathcal{H}_{\Omega}(z)
$$

Using the mean value theorem and recalling (4.16), one has

$$
\mathcal{P}_{3}^{(2 b)}(z)=\int_{0}^{1} \mathcal{P}_{\Omega}\left(\Psi_{X}^{0, \tau}(z), \tau\right) \mathrm{d} \tau,
$$

where for any $\tau \in[0,1]$, the Hamiltonian $\mathcal{P}_{\Omega}(z, \tau)$ is defined by

$$
\mathcal{P}_{\Omega}(z, \tau):=\left(\nabla \mathcal{H}_{\Omega}(z), X(z, \tau)\right)_{r}
$$

One has that

$$
\left(\nabla \mathcal{H}_{\Omega}(z), X(z, \tau)\right)_{r}=\frac{1}{2} \nabla_{S} \mathcal{H}_{\Omega}(z) \cdot \pi_{S} X(z, \tau)+\left(\Omega_{\perp}^{\mathrm{nls}}\left(I_{S}, 0\right) z_{\perp}, \pi_{\perp} X(z, \tau)\right)_{r}
$$

By (4.12), the vector field $X(z, \tau)$ was chosen to be

$$
X(z, \tau)=-\mathcal{L}_{\tau}(z)^{-1} E(z)
$$

where $E(z)$ is given by (4.10) and $\mathcal{L}_{\tau}(z)^{-1}$ by the Neumann series (4.6) in Lemma 4.1. Hence

$$
\begin{aligned}
X(z, \tau) & =-\mathcal{L}_{\tau}(z)^{-1} E(z)=-J E(z)-\sum_{n \geq 1}(-1)^{n} \tau^{n}(J L(z))^{n} J E(z) \\
& =-J E(z)+\tau J L(z) \sum_{n \geq 0}(-1)^{n} \tau^{n}(J L(z))^{n} J E(z) \\
& =-J E(z)+\tau J L(z) X(z, \tau) .
\end{aligned}
$$

Since $E=\Pi_{S} E$ and $J^{t}=-J$, the last term in (5.52) becomes

$$
\begin{aligned}
\left(\Omega_{\perp}^{\mathrm{nls}}\left(I_{S}, 0\right) z_{\perp}, \pi_{\perp} X(z, \tau)\right)_{r} & =\left(\Omega_{\perp}^{\mathrm{nls}}\left(I_{S}, 0\right) z_{\perp}, \pi_{\perp} \tau J L(z) X(z, \tau)\right)_{r} \\
& =-\tau\left(J \Omega_{\perp}^{\mathrm{nls}}\left(I_{S}, 0\right) z_{\perp}, \pi_{\perp} L(z) X(z, \tau)\right)_{r}
\end{aligned}
$$

By (3.40), the component $L_{\perp}^{\perp}(z)$ of $L(z)$ vanishes. Hence using the projections introduced in (5.43) and (5.44), one has

$$
\pi_{\perp} L(z) X(z, \tau)=L_{\perp}^{S}(z) \pi_{S} X(z, \tau) .
$$


Substituting the latter expression into (5.54) then leads to

$$
\begin{aligned}
\left(\Omega_{\perp}^{\mathrm{nls}}\left(I_{S}, 0\right) z_{\perp}, \pi_{\perp} X(z, \tau)\right)_{r} & =-\tau\left(J \Omega_{\perp}^{\mathrm{nls}}\left(I_{S}, 0\right) z_{\perp}, L_{\perp}^{S}(z) \pi_{S} X(z, \tau)\right)_{r} \\
& =-\tau L_{\perp}^{S}(z)^{t} J \Omega_{\perp}^{\mathrm{nls}}\left(I_{S}, 0\right) z_{\perp} \cdot \pi_{S} X(z, \tau) \\
& \stackrel{(3.46)}{=} \tau L_{S}^{\perp}(z) J \Omega_{\perp}^{\mathrm{nls}}\left(I_{S}, 0\right) z_{\perp} \cdot \pi_{S} X(z, \tau) .
\end{aligned}
$$

By the definition (3.42),

$$
L_{S}^{\perp}(z) J \Omega_{\perp}^{\mathrm{nls}}\left(I_{S}, 0\right) z_{\perp}=\left(\begin{array}{l}
\left(\mathrm{i}\left\langle J \mathrm{~d}_{\perp} \Psi^{\mathrm{nls}}\left(\Pi_{S} z\right)\left[J \Omega_{\perp}^{\mathrm{nls}}\left(I_{S}, 0\right) z_{\perp}\right], \partial_{x_{j}} \mathrm{~d}_{\perp} \Psi^{\mathrm{nls}}\left(\Pi_{S} z\right)\left[z_{\perp}\right]\right\rangle_{r}\right)_{j \in S} \\
\left(\mathrm{i}\left\langle J \mathrm{~d}_{\perp} \Psi^{\mathrm{nls}}\left(\Pi_{S} z\right)\left[J \Omega_{\perp}^{\mathrm{nls}}\left(I_{S}, 0\right) z_{\perp}\right], \partial_{Y_{j}} \mathrm{~d}_{\perp} \Psi^{\mathrm{nls}}\left(\Pi_{S} z\right)\left[z_{\perp}\right]\right\rangle_{r}\right)_{j \in S}
\end{array}\right) .
$$

Let us take a closer look at the expression

$$
\mathrm{d}_{\perp} \Psi^{\mathrm{nls}}\left(\Pi_{S} z\right)\left[J \Omega_{\perp}^{\mathrm{nls}}\left(I_{S}, 0\right) z_{\perp}\right]=\mathrm{d} \Psi^{\mathrm{nls}}\left(\Pi_{S} z\right)\left[J \Omega^{\mathrm{nls}}\left(I_{S}, 0\right)\left(0, z_{\perp}\right)\right] .
$$

Substituting for $J \Omega^{\text {nls }}\left(I_{S}, 0\right)\left(0, z_{\perp}\right)$ the right-hand side of the identity (5.25), one gets

$$
\begin{aligned}
\mathrm{d} \Psi^{\mathrm{nls}}\left(\Pi_{S} z\right)\left[J \Omega^{\mathrm{nls}}\left(I_{S}, 0\right)\left(0, z_{\perp}\right)\right] \\
=\mathrm{d} \Psi^{\mathrm{nls}}\left(\Pi_{S} z\right) J\left(\mathrm{~d} \Psi^{\mathrm{nls}}\left(\Pi_{S} z\right)\right)^{t} \mathrm{~d} \nabla \mathcal{H}^{\mathrm{nls}}\left(\Psi^{\mathrm{nls}}\left(\Pi_{S} z\right) \mathrm{d} \Psi^{\mathrm{nls}}\left(\Pi_{S} z\right)\left[\left(0, \widehat{z}_{\perp}\right)\right]\right. \\
\quad-\mathrm{d} \Psi^{\mathrm{nls}}\left(\Pi_{S} z\right) J \mathcal{R}^{(1)}\left(z_{S}\right)\left[\widehat{z}_{\perp}\right] .
\end{aligned}
$$

The first term on the right-hand side of the latter identity can be simplified. Since $\Psi^{\text {nls }}$ is symplectic,

$$
\mathrm{d} \Psi^{\mathrm{nls}}\left(\Pi_{S} z\right) J\left(\mathrm{~d} \Psi^{\mathrm{nls}}\left(\Pi_{S} z\right)\right)^{t}=\mathrm{i} \rrbracket
$$

one has

$$
\begin{aligned}
& \mathrm{d} \Psi^{\mathrm{nls}}\left(\Pi_{S} Z\right) J\left(\mathrm{~d} \Psi^{\mathrm{nls}}\left(\Pi_{S} z\right)\right)^{t} \mathrm{~d} \nabla \mathcal{H}^{\mathrm{nls}}\left(\Psi^{\mathrm{nls}}\left(\Pi_{S} z\right)\right) \mathrm{d} \Psi^{\mathrm{nls}}\left(\Pi_{S} z\right)\left[\left(0, \widehat{z}_{\perp}\right)\right] \\
& =\mathrm{i} \mathbb{d} \mathrm{d} \nabla \mathcal{H}^{\mathrm{nls}}\left(\Psi^{\mathrm{nls}}\left(\Pi_{S} z\right)\right) \mathrm{d} \Psi^{\mathrm{nls}}\left(\Pi_{S} z\right)\left[\left(0, z_{\perp}\right)\right]=\mathrm{i} \mathbb{J} \mathrm{d} \nabla \mathcal{H}^{\mathrm{nls}}\left(\Psi^{\mathrm{nls}}\left(\Pi_{S} z\right)\right) \mathrm{d}_{\perp} \Psi^{\mathrm{nls}}\left(\Pi_{S} z\right)\left[z_{\perp}\right] .
\end{aligned}
$$

Combining the above identities, the component $\mathrm{i}\left\langle\mathcal{J} \mathrm{d}_{\perp} \Psi^{\mathrm{nls}}\left(\Pi_{S} z\right)\left[J \Omega_{\perp}^{\mathrm{nls}}\left(I_{S}, 0\right) z_{\perp}\right]\right.$, $\left.\partial_{X_{j}} \mathrm{~d}_{\perp} \Psi^{\mathrm{nls}}\left(\Pi_{S} z\right)\left[z_{\perp}\right]\right\rangle_{r}$ on the right-hand side of (5.56) becomes, for $j \in S$ arbitrary,

$$
\begin{aligned}
\mathrm{i}\langle & \left.d \mathrm{~d}_{\perp} \Psi^{\mathrm{nls}}\left(\Pi_{S} z\right)\left[J \Omega_{\perp}^{\mathrm{nls}}\left(I_{S}, 0\right) z_{\perp}\right], \partial_{X_{j}} \mathrm{~d}_{\perp} \Psi^{\mathrm{nls}}\left(\Pi_{S} z\right)\left[z_{\perp}\right]\right\rangle_{r} \\
= & \left\langle\mathrm{d} \nabla \mathcal{H}^{\mathrm{nls}}\left(\Psi^{\mathrm{nls}}\left(\Pi_{S} z\right)\right) \mathrm{d}_{\perp} \Psi^{\mathrm{nls}}\left(\Pi_{S} z\right)\left[z_{\perp}\right], \partial_{x_{j}} \mathrm{~d}_{\perp} \Psi^{\mathrm{nls}}\left(\Pi_{S} z\right)\left[z_{\perp}\right]\right\rangle_{r} \\
& -\mathrm{i}\left\langle\int d \Psi^{\mathrm{nls}}\left(\Pi_{S} z\right) J \mathcal{R}^{(1)}\left(z_{S}\right)\left[z_{\perp}\right], \partial_{x_{j}} \mathrm{~d}_{\perp} \Psi^{\mathrm{nls}}\left(\Pi_{S} z\right)\left[z_{\perp}\right]\right\rangle_{r}
\end{aligned}
$$


which in view of $d \nabla \mathcal{H}^{\text {nls }}(w)=\mathcal{D}_{2}+d \nabla \mathcal{H}_{4}^{\text {nls }}(w)$ (cf. (5.33)) leads to

$$
\begin{aligned}
& \mathrm{i}\left\langle\int \mathrm{d}_{\perp} \Psi^{\mathrm{nls}}\left(\Pi_{S} z\right)\left[J \Omega_{\perp}^{\mathrm{nls}}\left(I_{S}, 0\right) z_{\perp}\right], \partial_{X_{j}} \mathrm{~d}_{\perp} \Psi^{\mathrm{nls}}\left(\Pi_{S} z\right)\left[z_{\perp}\right]\right\rangle_{r} \\
& =\left\langle\mathcal{D}_{2} \mathrm{~d}_{\perp} \Psi^{\mathrm{nls}}\left(z_{S}, 0\right)\left[z_{\perp}\right], \partial_{x_{j}} \mathrm{~d}_{\perp} \Psi^{\mathrm{nls}}\left(\Pi_{S} z\right)\left[z_{\perp}\right]\right\rangle_{r} \\
& +\left\langle\mathrm{d} \nabla \mathcal{H}_{4}^{\mathrm{nls}}\left(\Psi^{\mathrm{nls}}\left(\Pi_{S} Z\right)\right) \mathrm{d}_{\perp} \Psi^{\mathrm{nls}}\left(\Pi_{S} Z\right)\left[z_{\perp}\right], \partial_{x_{j}} \mathrm{~d}_{\perp} \Psi^{\mathrm{nls}}\left(\Pi_{S} Z\right)\left[z_{\perp}\right]\right\rangle_{r} \\
& -\mathrm{i}\left\langle\int \mathrm{d} d \Psi^{\mathrm{nls}}\left(\Pi_{S} z\right) J \mathcal{R}^{(1)}\left(z_{S}\right)\left[z_{\perp}\right], \partial_{X_{j}} \mathrm{~d}_{\perp} \Psi^{\mathrm{nls}}\left(\Pi_{S} z\right)\left[z_{\perp}\right]\right\rangle_{r} .
\end{aligned}
$$

Since $\mathcal{D}_{2}=\mathcal{D}_{2}^{t}$, the first term on the right-hand side on the latter identity can be written as

$$
\begin{aligned}
\left\langle\mathcal{D}_{2} \mathrm{~d}_{\perp} \Psi^{\mathrm{nls}}\left(\Pi_{S} z\right)\left[z_{\perp}\right],\right. & \left.\partial_{x_{j}} \mathrm{~d}_{\perp} \Psi^{\mathrm{nls}}\left(\Pi_{S} z\right)\left[z_{\perp}\right]\right\rangle_{r}=\frac{1}{2} \partial_{x_{j}}\left\langle\mathcal{D}_{2} \mathrm{~d}_{\perp} \Psi^{\mathrm{nls}}\left(\Pi_{S} z\right)\left[z_{\perp}\right], \mathrm{d}_{\perp} \Psi^{\mathrm{nls}}\left(\Pi_{S} z\right)\left[z_{\perp}\right]\right\rangle_{r} \\
& =\frac{1}{2} \partial_{x_{j}}\left\langle\mathcal{D}_{2} \mathrm{~d} \Psi^{\mathrm{nls}}\left(\Pi_{S} z\right)\left[\left(0, z_{\perp}\right)\right], \mathrm{d} \Psi^{\mathrm{nls}}\left(\Pi_{S} z\right)\left[\left(0, z_{\perp}\right)\right]\right\rangle_{r} \\
& =\frac{1}{2} \partial_{x_{j}}\left(\left(\mathrm{~d} \Psi^{\mathrm{nls}}\left(\Pi_{S} z\right)\right)^{t} \mathcal{D}_{2} \mathrm{~d} \Psi^{\mathrm{nls}}\left(\Pi_{S} z\right)\left[\left(0, z_{\perp}\right)\right],\left(0, z_{\perp}\right)\right)_{r^{\prime}}
\end{aligned}
$$

which can be further transformed as follows: using $\mathcal{D}_{2}=\mathrm{d} \nabla \mathcal{H}^{\text {nls }}-\mathrm{d} \nabla \mathcal{H}_{4}^{\text {nls }}$ (cf. (5.33)) and taking into account that by (5.25),

$$
\left(\mathrm{d} \Psi^{\mathrm{nls}}\left(z_{S}, 0\right)\right)^{t} \mathrm{~d} \nabla \mathcal{H}^{\mathrm{nls}}\left(\Psi^{\mathrm{nls}}\left(z_{S}, 0\right)\right) \mathrm{d} \Psi^{\mathrm{nls}}\left(z_{S}, 0\right)\left[\left(0, z_{\perp}\right)\right]=\Omega^{\mathrm{nls}}\left(I_{S}, 0\right)\left[\left(0, z_{\perp}\right)\right]+\mathcal{R}^{(1)}\left(z_{S}\right)\left[z_{\perp}\right]
$$

one is lead to

$$
\begin{aligned}
& \frac{1}{2} \partial_{X_{j}}\left(\left(\mathrm{~d} \Psi^{\mathrm{nls}}\left(\Pi_{S} z\right)\right)^{t} \mathcal{D}_{2} \mathrm{~d} \Psi^{\mathrm{nls}}\left(\Pi_{S} z\right)\left[\left(0, z_{\perp}\right)\right],\left(0, z_{\perp}\right)\right)_{r}=\frac{1}{2} \partial_{x_{j}}\left(\Omega^{\mathrm{nls}}\left(I_{S}, 0\right)\left[\left(0, z_{\perp}\right)\right],\left(0, z_{\perp}\right)\right)_{r} \\
& \left.+\frac{1}{2} \partial_{x_{j}}\left(\mathcal{R}^{(1)}\left(z_{S}\right)\left[z_{\perp}\right],\left(0, z_{\perp}\right)\right)_{r}-\frac{1}{2} \partial_{X_{j}}\left\langle\mathrm{~d} \nabla \mathcal{H}_{4}^{\mathrm{nls}}\left(\Psi^{\mathrm{nls}}\left(\Pi_{S} z\right)\right)\right] \mathrm{d}_{\perp} \Psi^{\mathrm{nls}}\left(z_{S}, 0\right)\left[z_{\perp}\right], \mathrm{d}_{\perp} \Psi^{\mathrm{nls}}\left(\Pi_{S} z\right)\left[z_{\perp}\right]\right\rangle_{r}
\end{aligned}
$$

Let us analyse $\partial_{x_{j}}\left(\Omega^{\text {nls }}\left(I_{S}, 0\right)\left[\left(0, z_{\perp}\right)\right],\left(0, z_{\perp}\right)\right)_{r}=\left(\partial_{x_{j}} \Omega_{\perp}^{\text {nls }}\left(I_{S}, 0\right) z_{\perp}, z_{\perp}\right)_{r}$ in more detail. Substituting for $\Omega_{\perp}^{\text {nls }}\left(I_{S}, 0\right)$ the expression $D_{\perp}^{2}+\Omega_{\perp}^{(0)}\left(I_{S}, 0\right)$ (cf. (5.27)) and using that $\left(\partial_{x_{j}} D_{\perp}^{2} z_{\perp}, z_{\perp}\right)_{r}=0$ for any $j \in S$, one concludes that

$$
\left(\partial_{x_{j}} \Omega_{\perp}^{\mathrm{nls}}\left(I_{S}, 0\right) z_{\perp}, z_{\perp}\right)_{r}=\left(\partial_{x_{j}} \Omega_{\perp}^{(0)}\left(I_{S}, 0\right) z_{\perp}, z_{\perp}\right)_{r^{\prime}} \quad \forall j \in S
$$

The above identities then imply that (5.60) becomes

$$
\begin{aligned}
& \left\langle\mathcal{D}_{2} \mathrm{~d}_{\perp} \Psi^{\mathrm{nls}}\left(\Pi_{S} z\right)\left[z_{\perp}\right], \partial_{x_{j}} \mathrm{~d}_{\perp} \Psi^{\mathrm{nls}}\left(\Pi_{S} z\right)\left[z_{\perp}\right]\right\rangle_{r}=\frac{1}{2}\left(\partial_{X_{j}} \Omega_{\perp}^{(0)}\left(I_{S}, 0\right) z_{\perp}, z_{\perp}\right)_{r} \\
& +\frac{1}{2}\left(\partial_{X_{j}} \mathcal{R}^{(1)}\left(w_{S}\right) z_{\perp},\left(0, z_{\perp}\right)\right)_{r}-\frac{1}{2} \partial_{X_{j}}\left\langle\mathrm{~d} \nabla \mathcal{H}_{4}^{\mathrm{nls}}\left(\Psi^{\mathrm{nls}}\left(\Pi_{S} z\right)\right) \mathrm{d}_{\perp} \Psi^{\mathrm{nls}}\left(\Pi_{S} z\right)\left[z_{\perp}\right], \mathrm{d}_{\perp} \Psi^{\mathrm{nls}}\left(\Pi_{S} z\right)\left[z_{\perp}\right]\right\rangle_{r} .
\end{aligned}
$$


With (5.60) and (5.61), the identity (5.59) becomes

$$
\mathrm{i}\left\langle\int \mathrm{d}_{\perp} \Psi^{\mathrm{nls}}\left(\Pi_{S} z\right)\left[J \Omega_{\perp}^{\mathrm{nls}}\left(I_{S}, 0\right) z_{\perp}\right], \partial_{x_{j}} \mathrm{~d}_{\perp} \Psi^{\mathrm{nls}}\left(\Pi_{S} z\right)\left[z_{\perp}\right]\right\rangle_{r}=\left(\mathcal{R}_{x_{j}}\left(z_{S}\right)\left[z_{\perp}\right], z_{\perp}\right)_{r^{\prime}}
$$

where for any $j \in S, \mathcal{R}_{X_{j}}\left(z_{S}\right): h_{\perp c}^{0} \rightarrow h_{\perp c}^{0}$ is the linear operator defined by

$$
\begin{aligned}
& \frac{1}{2} \partial_{x_{j}} \Omega_{\perp}^{(0)}\left(I_{S}, 0\right)+\frac{1}{2} \pi_{\perp} \partial_{x_{j}} \mathcal{R}^{(1)}\left(z_{S}\right)-\frac{1}{2} \partial_{x_{j}}\left(\left(\mathrm{~d}_{\perp} \Psi^{\mathrm{nls}}\left(z_{S}, 0\right)\right)^{t} \mathrm{~d} \nabla \mathcal{H}_{4}^{\mathrm{nls}}\left(\Psi^{\mathrm{nls}}\left(\Pi_{S} z\right)\right) \mathrm{d}_{\perp} \Psi^{\mathrm{nls}}\left(\Pi_{S} z\right)\right) \\
& +\left(\partial_{x_{j}} \mathrm{~d}_{\perp} \Psi^{\mathrm{nls}}\left(\Pi_{S} z\right)\right)^{t} \mathrm{~d} \nabla \mathcal{H}_{4}^{\mathrm{nls}}\left(\Psi^{\mathrm{nls}}\left(\Pi_{S} z\right)\right) \mathrm{d}_{\perp} \Psi^{\mathrm{nls}}\left(\Pi_{S} z\right) \\
& -\mathrm{i}\left(\partial_{X_{j}} \mathrm{~d}_{\perp} \Psi^{\mathrm{nls}}\left(\Pi_{S} z\right)\right)^{t} \mathbb{J} \mathrm{d} \Psi^{\mathrm{nls}}\left(\Pi_{S} z\right) J \mathcal{R}^{(1)}\left(z_{S}\right) \text {. }
\end{aligned}
$$

Arguing similarly as above one obtains

$$
\mathrm{i}\left\langle J \mathrm{~d}_{\perp} \Psi^{\mathrm{nls}}\left(\Pi_{S} z\right)\left[J \Omega_{\perp}^{\mathrm{nls}}\left(I_{S}, 0\right) z_{\perp}\right], \partial_{Y_{j}} \mathrm{~d}_{\perp} \Psi^{\mathrm{nls}}\left(\Pi_{S} z\right)\left[z_{\perp}\right]\right\rangle_{r}=\left(\mathcal{R}_{Y_{j}}\left(z_{S}\right)\left[z_{\perp}\right], z_{\perp}\right)_{r^{\prime}}
$$

where $\mathcal{R}_{Y_{j}}\left(z_{S}\right): h_{\perp c}^{0} \rightarrow h_{\perp c}^{0}$ is given by

$$
\begin{aligned}
& \frac{1}{2} \partial_{Y_{j}} \Omega_{\perp}^{(0)}\left(I_{S}, 0\right)+\frac{1}{2} \pi_{\perp} \partial_{Y_{j}} \mathcal{R}^{(1)}\left(z_{S}\right)-\frac{1}{2} \partial_{Y_{j}}\left(\left(\mathrm{~d}_{\perp} \Psi^{\mathrm{nls}}\left(z_{S}, 0\right)\right)^{t} \mathrm{~d} \nabla \mathcal{H}_{4}^{\mathrm{nls}}\left(\Psi^{\mathrm{nls}}\left(\Pi_{S} Z\right)\right) \mathrm{d}_{\perp} \Psi^{\mathrm{nls}}\left(\Pi_{S} Z\right)\right)+ \\
& \left(\partial_{Y_{j}} \mathrm{~d}_{\perp} \Psi^{\mathrm{nls}}\left(\Pi_{S} Z\right)\right)^{t} \mathrm{~d} \nabla \mathcal{H}_{4}^{\mathrm{nls}}\left(\Psi^{\mathrm{nls}}\left(\Pi_{S} z\right)\right) \mathrm{d}_{\perp} \Psi^{\mathrm{nls}}\left(\Pi_{S} Z\right)-\mathrm{i}\left(\partial_{Y_{j}} \mathrm{~d}_{\perp} \Psi^{\mathrm{nls}}\left(\Pi_{S} Z\right)\right)^{t} J \mathrm{~d} \Psi^{\mathrm{nls}}\left(\Pi_{S} Z\right) J \mathcal{R}^{(1)}\left(z_{S}\right)
\end{aligned}
$$

In the next lemma we state estimates for the operators $\mathcal{R}_{X_{j}}\left(z_{S}\right)$ and $\mathcal{R}_{Y_{j}}\left(z_{S}\right)$.

Lemma 5.5. For any $j \in S$ and $s \in \mathbb{Z}_{\geq 0}$, the maps

$$
\begin{gathered}
\mathcal{R}_{x_{j}}: \mathcal{V}_{S} \cap\left(\mathbb{R}^{S} \times \mathbb{R}^{S}\right) \rightarrow \mathcal{L}\left(h_{\perp c}^{s}, h_{\perp c}^{s}\right), z_{S} \mapsto \mathcal{R}_{X_{j}}\left(z_{S}\right), \\
\mathcal{R}_{Y_{j}}: \mathcal{V}_{S} \cap\left(\mathbb{R}^{S} \times \mathbb{R}^{S}\right) \rightarrow \mathcal{L}\left(h_{\perp c}^{s}, h_{\perp c}^{s}\right), z_{S} \mapsto \mathcal{R}_{Y_{j}}\left(z_{S}\right)
\end{gathered}
$$

are real analytic and bounded. Furthermore, for any $\alpha, \beta \in \mathbb{Z}_{\geq 0}^{S}$,

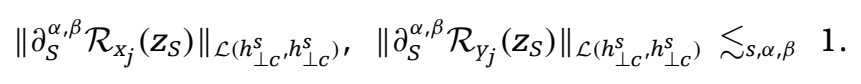

Proof. The lemma follows from Theorem 1.1 and Lemmata 5.1, 5.2.

Finally, by (5.51), (5.52), (5.55), (5.56), (5.62), (5.64) and writing

$$
\pi_{S} X(z, \tau)=\left(\left(X_{j,+}(z, \tau)\right)_{j \in S^{\prime}}\left(X_{j,-}(z, \tau)\right)_{j \in S}\right) \in \mathbb{R}^{S} \times \mathbb{R}^{S}
$$


one sees that the Hamiltonian $\mathcal{P}_{\Omega}(z, \tau)$, defined by (5.51), can be written in the form

$$
\frac{1}{2} \nabla_{S} \mathcal{H}_{\Omega}(z) \cdot \pi_{S} X(z, \tau)+\sum_{j \in S} X_{j,+}(z, \tau)\left(\mathcal{R}_{X_{j}}\left(z_{S}\right)\left[z_{\perp}\right], z_{\perp}\right)_{r}+\sum_{j \in S} X_{j,-}(z, \tau)\left(\mathcal{R}_{Y_{j}}\left(z_{S}\right)\left[z_{\perp}\right], z_{\perp}\right)_{r} .
$$

In the next lemma we state estimates for the Hamiltonian $\mathcal{P}_{3}^{(2 b)}$, defined in (5.50).

Lemma 5.6. For any $s \in \mathbb{Z}_{\geq 0}$, the Hamiltonian $\mathcal{P}_{3}^{(2 b)}: \mathcal{V}_{\delta}^{\prime} \cap h_{r}^{s} \rightarrow \mathbb{R}$ is real analytic. Moreover, it satisfies the following tame estimates: for any $s \in \mathbb{Z}_{\geq 0}, z \in \mathcal{V}_{\delta}^{\prime} \cap h_{r}^{s}, \widehat{z}, \widehat{z}_{1}, \widehat{z}_{2} \in$ $h_{c^{\prime}}^{s}$

$$
\begin{gathered}
\left\|\nabla \mathcal{P}_{3}^{(2 b)}(z)\right\|_{s} \lesssim_{s}\left\|z_{\perp}\right\|_{s}\left\|z_{\perp}\right\|_{0}^{2}, \quad\left\|\mathrm{~d} \nabla \mathcal{P}_{3}^{(2 b)}(z)[\widehat{z}]\right\|_{s} \lesssim_{s}\left\|z_{\perp}\right\|_{s}\left\|z_{\perp}\right\|_{0}\|\widehat{Z}\|_{0}+\left\|z_{\perp}\right\|_{0}^{2}\|\widehat{\boldsymbol{Z}}\|_{s}, \\
\left\|\mathrm{~d}^{2} \nabla \mathcal{P}_{3}^{(2 b)}(z)\left[\widehat{Z}_{1}, \widehat{Z}_{2}\right]\right\|_{s} \lesssim_{s}\left\|z_{\perp}\right\|_{s}\left\|\widehat{Z}_{1}\right\|_{0}\left\|\widehat{Z}_{2}\right\|_{0}+\left\|z_{\perp}\right\|_{0}\left(\left\|\widehat{Z}_{1}\right\|_{s}\left\|\widehat{Z}_{2}\right\|_{0}+\left\|\widehat{Z}_{1}\right\|_{0}\left\|\widehat{Z}_{2}\right\|_{s}\right),
\end{gathered}
$$

and for any $k \in \mathbb{Z}_{\geq 3}, \widehat{z}_{1}, \ldots, \widehat{z}_{k} \in h_{c}^{s}$

$$
\left\|\mathrm{d}^{k} \nabla \mathcal{P}_{3}^{(2 b)}(z)\left[\widehat{z}_{1}, \ldots, \widehat{z}_{k}\right]\right\|_{s+1} \lesssim s, k \sum_{j=1}^{k}\left\|\widehat{Z}_{j}\right\|_{s} \prod_{i \neq j}\left\|\widehat{Z}_{i}\right\|_{0}+\left\|z_{\perp}\right\|_{s} \prod_{j=1}^{k}\left\|\widehat{Z}_{j}\right\|_{0}
$$

Proof. The lemma follows by (5.50), (5.66), and Lemmata 4.3, 4.4, 5.2, 5.5.

5.2.2.3 Term $\mathcal{P}_{2}^{(1)}$. Recall that the Hamiltonian $\mathcal{P}_{2}^{(1)}$ was introduced in (5.42). For $z \in$ $\mathcal{V}_{\delta}^{\prime} \cap h_{r}^{0}$ one has $\Psi_{C}(z)=z+B_{C}(z)$ and hence the Taylor expansion of $\mathcal{P}_{2}^{(1)}\left(\Psi_{C}(z)\right)$ around $z$ reads

$$
\mathcal{P}_{2}^{(1)}\left(\Psi_{C}(z)\right)=\mathcal{P}_{2}^{(1)}(z)+\mathcal{P}_{3}^{(2 c)}(z), \quad \mathcal{P}_{3}^{(2 c)}(z):=\int_{0}^{1}\left(\nabla \mathcal{P}_{2}^{(1)}\left(z+t B_{C}(z)\right), B_{C}(z)\right)_{r} \mathrm{~d} t .
$$

The following lemma holds:

Lemma 5.7. For any $s \in \mathbb{Z}_{\geq 0}$, the Hamiltonian $\mathcal{P}_{2}^{(1)} \circ \Psi_{C}: \mathcal{V}_{\delta}^{\prime} \cap h_{r}^{s} \rightarrow \mathbb{R}$ is real analytic. Moreover, the Hamiltonian $\mathcal{P}_{3}^{(2 c)}$, defined in (5.67), satisfies the following tame estimates: for any $s \in \mathbb{Z}_{\geq 0}, z \in \mathcal{V}_{\delta}^{\prime} \cap h_{r}^{s}, \widehat{z} \in h_{c^{\prime}}^{s}$

$$
\left\|\nabla \mathcal{P}_{3}^{(2 c)}(z)\right\|_{s} \lesssim_{s}\left\|z_{\perp}\right\|_{s}\left\|z_{\perp}\right\|_{0}, \quad\left\|\mathrm{~d} \nabla \mathcal{P}_{3}^{(2 c)}(z)[\widehat{z}]\right\|_{s} \lesssim_{s}\left\|z_{\perp}\right\|_{s}\|\widehat{Z}\|_{0}+\left\|z_{\perp}\right\|_{0}\|\widehat{Z}\|_{s}
$$

and for any $k \in \mathbb{Z}_{\geq 2}, \widehat{z}_{1}, \ldots, \widehat{z}_{k} \in h_{c}^{s}$,

$$
\left\|\mathrm{d}^{k} \nabla \mathcal{P}_{3}^{(2 c)}(\boldsymbol{z})\left[\widehat{\boldsymbol{z}}_{1}, \ldots, \widehat{\boldsymbol{z}}_{k}\right]\right\|_{s} \lesssim s, k \sum_{j=1}^{k}\left\|\widehat{\boldsymbol{Z}}_{j}\right\|_{s} \prod_{i \neq j}\left\|\widehat{\boldsymbol{z}}_{i}\right\|_{0}+\left\|\boldsymbol{z}_{\perp}\right\|_{s} \prod_{j=1}^{k}\left\|\widehat{\boldsymbol{z}}_{j}\right\|_{0}
$$


Proof. The lemma follows by differentiating $\mathcal{P}_{3}^{(2 c)}$ and applying Corollary 4.1 and Lemma 5.3(i).

5.2.2.4 Term $\mathcal{P}_{3}^{(1)}$. By $(5.42), \mathcal{P}_{3}^{(1)}$ is given by $\mathcal{T}_{3}^{(1)}\left(z_{S}, \mathrm{~d} \Psi^{\mathrm{nls}}\left(\Pi_{S} z\right)\left[\Pi_{\perp} z\right]\right)$ where $\mathcal{T}_{3}^{(1)}$ is the Taylor remainder term of order three, introduced in (5.35). Using the estimates of $\mathcal{P}_{3}^{(1)}$ of Lemma 5.3(ii), the Hamiltonian $\mathcal{P}_{3}^{(1)} \circ \Psi_{C}$ can be estimated as follows:

Lemma 5.8. For any $s \in \mathbb{Z}_{\geq 0}, \mathcal{P}_{3}^{(1)} \circ \Psi_{C}: \mathcal{V}_{\delta}^{\prime} \cap h_{r}^{s} \rightarrow \mathbb{R}$ is real analytic. Moreover, the following tame estimates hold: for any $s \in \mathbb{Z}_{\geq 1}, z \in \mathcal{V}_{\delta}^{\prime} \cap h_{r}^{s}, \widehat{z} \in h_{c^{\prime}}^{s}$,

$$
\left\|\nabla\left(\mathcal{P}_{3}^{(1)} \circ \Psi_{C}\right)(z)\right\|_{s} \lesssim_{s}\left\|z_{\perp}\right\|_{s}\left\|z_{\perp}\right\|_{0}, \quad\left\|\mathrm{~d} \nabla\left(\mathcal{P}_{3}^{(1)} \circ \Psi_{C}\right)(z)[\widehat{Z}]\right\|_{s} \lesssim_{s}\left\|z_{\perp}\right\|_{s}\|\widehat{Z}\|_{0}+\left\|z_{\perp}\right\|_{0}\|\widehat{Z}\|_{s},
$$

and for any $k \in \mathbb{Z}_{\geq 2}, \widehat{z}_{1}, \ldots, \widehat{z}_{k} \in h_{c}^{s}$,

$$
\left\|\mathrm{d}^{k} \nabla\left(\mathcal{P}_{3}^{(1)} \circ \Psi_{C}\right)(z)\left[\widehat{Z}_{1}, \ldots, \widehat{\boldsymbol{Z}}_{k}\right]\right\|_{s} \lesssim s, k \sum_{j=1}^{k}\left\|\widehat{\boldsymbol{Z}}_{j}\right\|_{s} \prod_{i \neq j}\left\|\widehat{\boldsymbol{Z}}_{i}\right\|_{0}+\left\|\boldsymbol{z}_{\perp}\right\|_{s} \prod_{j=1}^{k}\left\|\widehat{\boldsymbol{Z}}_{j}\right\|_{0} .
$$

Proof. The lemma follows by differentiating the Hamiltonian $\mathcal{P}_{3}^{(1)} \circ \Psi_{C}$ and applying Corollary 4.1 and Lemma 5.3(ii).

By (5.41), (5.46), (5.49), (5.67) one gets that the Hamiltonian $\mathcal{H}^{(2)}:=\mathcal{H}^{(1)} \circ \Psi_{C}=\mathcal{H}^{\text {nls }} \circ \Psi_{L} \circ \Psi_{C}$ has the form

$$
\mathcal{H}^{(2)}(z)=H^{\mathrm{nls}}\left(I_{S}, 0\right)+\frac{1}{2}\left(\Omega_{\perp}^{\mathrm{nls}}\left(I_{S}, 0\right)\left[z_{\perp}\right], z_{\perp}\right)_{r}+\mathcal{P}_{2}(z)+\mathcal{P}_{3}(z)
$$

where for any $z \in \mathcal{V}_{\delta}^{\prime} \cap h_{r}^{0}$,

$$
\begin{array}{r}
\mathcal{P}_{2}(z):=\nabla_{S} h^{\mathrm{nls}}\left(z_{S}\right) \cdot \pi_{S} B_{2}^{C}(z)+\mathcal{P}_{2}^{(1)}(z), \\
\mathcal{P}_{3}(z):=\mathcal{P}_{3}^{(2 a)}(z)+\mathcal{P}_{3}^{(2 b)}(z)+\mathcal{P}_{3}^{(2 c)}(z)+\mathcal{P}_{3}^{(1)}\left(\Psi_{C}(z)\right) .
\end{array}
$$

Note that $\mathcal{P}_{2}$ is quadratic with respect to $z_{\perp}$, whereas $\mathcal{P}_{3}$ is a remainder term of order three in $z_{\perp}$. Being quadratic with respect to $z_{\perp}, \mathcal{P}_{2}$ can be written as

$$
\mathcal{P}_{2}(z)=\frac{1}{2}\left(\mathrm{~d}_{\perp}\left(\nabla_{\perp} \mathcal{P}_{2}\left(\Pi_{S} z\right)\right)\left[z_{\perp}\right], z_{\perp}\right)_{r}
$$

We prove the following

Lemma 5.9. The Hamiltonian $\mathcal{P}_{2}$ vanishes on $\mathcal{V}_{\delta}^{\prime} \cap h_{r}^{0}$. 
Proof. By Corollary 4.1, $\Psi_{C}\left(\Pi_{S} z\right)=\Pi_{S} z$ and $\mathrm{d} \Psi_{C}\left(\Pi_{S} z\right)=\mathrm{Id}$. Hence by the chain rule and formula (3.5), the map $\Psi=\Psi_{L} \circ \Psi_{C}$ satisfies

$$
\mathrm{d} \Psi\left(\Pi_{S} z\right)=\mathrm{d} \Psi_{L}\left(\Pi_{S} z\right)=\mathrm{d} \Psi^{\mathrm{nls}}\left(\Pi_{S} z\right)
$$

Recall that we denoted by $\widehat{w}(t)$ the solution of equation (5.20), obtained by linearizing the dNLS equation along $w(t)=\Psi^{\text {nls }}\left(\Pi_{S} z(t)\right)$ with initial data $\widehat{W}(0)=\mathrm{d} \Psi^{(n l s)}\left(\Pi_{S} z(t)\right)\left(0, \widehat{z}_{\perp}^{(0)}\right)$ and by $\widehat{z}(t)=\left(0, \widehat{z}_{\perp}(t)\right)$ the one of the equations obtained by linearizing the dNLS equation, expressed in Birkhoff coordinates (cf. (5.14)), along $\left(z_{S}(t), 0\right)=\Pi_{S} z(t)$ with initial data $\left(0, \widehat{z}_{\perp}^{(0)}\right)$. Since $\Psi^{\mathrm{nls}}$ is symplectic, $\widehat{W}(t)=\mathrm{d} \Psi^{\mathrm{nls}}\left(\Pi_{S} z(t)\right)[\widehat{z}(t)]$. We remark that $\left(z_{S}(t), 0\right)=\Pi_{S} z(t)$ is also a solution of the Hamiltonian equation $\partial_{t} z^{(2)}=J \nabla \mathcal{H}^{(2)}\left(z^{(2)}\right)$ with $\mathcal{H}^{(2)}$ given by (5.68). Denote by $\widehat{Z}^{(2)}(t)=\left(0, \widehat{z}_{\perp}^{(2)}(t)\right)$ the solution of the equation obtained by linearizing $\partial_{t} z^{(2)}=J \nabla \mathcal{H}^{(2)}\left(z^{(2)}\right)$ along $\Pi_{S} z(t)$ with the same initial data $\left(0, \widehat{z}_{\perp}^{(0)}\right)$ as above. Since $\Psi$ is symplectic, $\widehat{W}(t)=\mathrm{d} \Psi\left(\Pi_{S} Z(t)\right)\left[\widehat{Z}^{(2)}(t)\right]$, implying together with $\mathrm{d} \Psi\left(\Pi_{S} z\right)=\mathrm{d} \Psi^{\mathrm{nls}}\left(\Pi_{S} z\right)$ (cf. (5.71) above) that $\widehat{z}^{(2)}(t)=\widehat{z}(t)$ for any $t$. By $(5.18), \widehat{z}_{\perp}(t)$ satisfies

$$
\partial_{t} \widehat{z}_{\perp}(t)=J \Omega_{\perp}^{\mathrm{nls}}\left(I_{S}, 0\right)\left[\widehat{z}_{\perp}(t)\right]
$$

whereas by (5.68), one has

$$
\partial_{t} \widehat{z}_{\perp}^{(2)}(t)=J \mathrm{~d}_{\perp} \nabla \mathcal{H}^{(2)}\left(\Pi_{S} z(t)\right)\left[z_{\perp}^{(2)}(t)\right]=J \Omega_{\perp}^{\mathrm{nls}}\left(I_{S}, 0\right)\left[\widehat{z}_{\perp}^{(2)}(t)\right]+J \mathrm{~d}_{\perp} \nabla_{\perp} \mathcal{P}_{2}\left(\Pi_{S} z(t)\right)\left[\widehat{z}_{\perp}^{(2)}(t)\right]
$$

In particular, it follows that $\mathrm{d}_{\perp} \nabla_{\perp} \mathcal{P}_{2}\left(\Pi_{S} z(0)\right)\left[\widehat{z}_{\perp}^{(0)}\right]=0$. Since $\mathcal{P}_{2}(z)$ is quadratic in $z_{\perp}$ and the initial data $z_{S}(0) \in \pi_{S}\left(\mathcal{V}_{\delta}^{\prime} \cap h_{r}^{0}\right), \widehat{z}_{\perp}^{(0)} \in h_{\perp c}^{0}$ are arbitrary, it follows that $\mathcal{P}_{2}(z)=0$ for any $z \in \mathcal{V}_{\delta}^{\prime} \cap h_{r}^{0}$, which proves the claimed statement.

As a consequence of Lemma 5.9, formula (5.68) becomes

$$
\mathcal{H}^{(2)}(z)=H^{\mathrm{nls}}\left(I_{S}, 0\right)+\frac{1}{2}\left(\Omega_{\perp}^{\mathrm{nls}}\left(I_{S}, 0\right)\left[z_{\perp}\right], z_{\perp}\right)_{r}+\mathcal{P}_{3}(z)
$$

The Hamiltonian $\mathcal{P}_{3}$, introduced in (5.70), satisfies the following tame estimates.

Lemma 5.10 (Tame estimates of $\mathcal{P}_{3}$ ). For any $s \in \mathbb{Z}_{\geq 0}$, the Hamiltonian $\mathcal{P}_{3}: \mathcal{V}_{\delta}^{\prime} \cap h_{r}^{s} \rightarrow \mathbb{R}$ is real analytic and satisfies the following tame estimates: for any $z \in \mathcal{V}_{\delta}^{\prime} \cap h_{r}^{s}, \widehat{z} \in h_{c^{\prime}}^{s}$

$$
\left\|\nabla \mathcal{P}_{3}(z)\right\|_{s} \lesssim_{s}\left\|z_{\perp}\right\|_{s}\left\|z_{\perp}\right\|_{0}, \quad\left\|\mathrm{~d} \nabla \mathcal{P}_{3}(z)[\widehat{z}]\right\|_{s} \lesssim_{s}\left\|z_{\perp}\right\|_{s}\|\widehat{Z}\|_{0}+\left\|z_{\perp}\right\|_{0}\|\widehat{Z}\|_{s}
$$


1522 T. Kappeler and R. Montalto

and for any $k \in \mathbb{Z}_{\geq 2}, \widehat{z}_{1}, \ldots, \widehat{z}_{k} \in h_{c}^{s}$,

$$
\left\|\mathrm{d}^{k} \nabla \mathcal{P}_{3}(z)\left[\widehat{z}_{1}, \ldots, \widehat{z}_{k}\right]\right\|_{s} \lesssim s \sum_{j=1}^{k}\left\|\widehat{z}_{j}\right\|_{s} \prod_{i \neq j}\left\|\widehat{z}_{i}\right\|_{0}+\left\|z_{\perp}\right\|_{s} \prod_{j=1}^{k}\left\|\widehat{z}_{j}\right\|_{0} .
$$

Proof. The claimed statements follow from Lemmata 5.4 and 5.6-5.8.

\subsection{Summary of the proof of Theorem 1.2}

Theorem 1.2 is a direct consequence of Propositions 5.1, 5.2, formula (5.74), and Lemma 5.10. The neighbourhood $\mathcal{V}$ in the statement of the theorem is given by $\mathcal{V}_{\delta}^{\prime}$ introduced in (4.16).

\section{Proof of Theorem 1.3}

Within this proof, it is convenient to use complex Birkhoff coordinates, given by $\zeta_{n}:=$ $\left(x_{n}-\mathrm{i} y_{n}\right) / \sqrt{2}, n \in \mathbb{Z}$. A solution $z(t)=(x(t), y(t))$ of the dNLS equation in Birkhoff coordinates then satisfies the equations

$$
\partial_{t} \zeta_{n}=-\mathrm{i} \omega_{n}^{\mathrm{nls}} \zeta_{n}, \quad n \in \mathbb{Z}
$$

where

$$
\omega_{n}^{\mathrm{nls}} \equiv \omega_{n}^{\mathrm{nls}}\left(I_{S}, I_{\perp}\right)=\partial_{I_{n}} H^{\mathrm{nls}}\left(I_{S}, I_{\perp}\right)
$$

Linearize (6.1) at a solution $\zeta(t)$ of the form $\left(\zeta_{S}(t), 0\right)$. For initial data of the form $\widehat{\zeta}(0)=\left(0, \widehat{\zeta}_{\perp}(0)\right)$, the corresponding solution $\widehat{\zeta}(t)=\left(\widehat{\zeta}_{S}(t), \widehat{\zeta}_{\perp}(t)\right)$ of the linearized equation satisfies

$$
\widehat{\zeta}_{S}(t) \equiv 0, \quad \partial_{t} \widehat{\zeta}_{n}(t)=-\mathrm{i} \omega_{n}^{\mathrm{nls}}\left(I_{S}, 0\right) \widehat{\zeta}_{n}(t), \quad n \in S^{\perp}
$$

The latter equation is reduced to constant coefficients and hence

$$
\widehat{\zeta}_{\perp}(t)=\left(\mathrm{e}^{-\mathrm{i} \omega_{n}\left(I_{S}, 0\right) t} \widehat{\zeta}_{n}(0)\right)_{n \in S^{\perp}}
$$

Since $\Psi^{\text {nls }}$ is symplectic, the solution of the equation, obtained by linearizing the dNLS equation along $\Psi^{\text {nls }}\left(z_{S}(t), 0\right)$, with initial data $d \Psi^{\text {nls }}\left(0, \widehat{\zeta}_{\perp}(0)\right)$, is given by

$$
\widehat{W}(t)=\mathrm{d} \Psi^{\mathrm{nls}}\left(z_{S}(t), 0\right)\left[0, \widehat{\zeta}_{\perp}(t)\right]
$$


We now consider the special solutions $\widehat{\zeta}^{ \pm, j}(t)=\mathrm{e}^{ \pm \mathrm{i} \omega_{j}\left(I_{S}, 0\right) t} \widehat{\zeta}^{ \pm, j}(0), j \in S^{\perp}$, corresponding to the initial data

$$
\widehat{\zeta}^{ \pm j}(0)=\left(\mathrm{e}^{(1, j)} \pm \mathrm{i}^{(2, j)}\right) / \sqrt{2}, \quad \mathrm{e}^{(1, j)}=\left(\left(\delta_{n j}\right)_{n \in \mathbb{Z}}, 0\right), \quad \mathrm{e}^{(2, j)}=\left(0,\left(\delta_{n j}\right)_{n \in \mathbb{Z}}\right) .
$$

These solutions are periodic in time and that $d \Psi^{\mathrm{nls}}(z(t))\left[\widehat{\zeta}^{ \pm, j}(t)\right]$ can be written as

$$
\widehat{W}^{ \pm, j}(t)=\mathrm{e}^{ \pm \mathrm{i} \omega_{j}\left(I_{S}, 0\right) t} \mathrm{~d} \Psi^{\mathrm{nls}}\left(z_{S}(t), 0\right)\left[\widehat{\zeta}^{ \pm, j}(0)\right]
$$

In the terminology of [16], $\widehat{W}^{+, j}(t), \widehat{w}^{-, j}(t), j \in S^{\perp}$, are Floquet solutions with Floquet exponents $\pm \omega_{j}\left(I_{S}, 0\right)$. By Theorem 1.1 one then concludes that up to normalizations (cf. Appendix 2) and natural identifications (such as the identifications of action angle with Birkhoff coordinates), the map $\Phi_{1}$, obtained by applying the scheme of construction of [16] to the dNLS equation, coincides with the map

$$
\mathbb{R}^{S} \times \mathbb{R}^{S} \rightarrow \mathcal{L}\left(h_{\perp r}^{s}, H_{r}^{s}\right),\left.\quad z_{S} \mapsto \mathrm{d} \Psi^{\mathrm{nls}}\left(z_{S}, 0\right)\right|_{h_{\perp r}^{s}}
$$

Since according to [16], the map $\Phi(z)$ can be chosen of the form $\Psi^{\text {nls }}\left(z_{S}, 0\right)+\Phi_{1}(z)$ and since the symplectic corrector $\Psi_{C}$ is constructed following the scheme in [16], one concludes that again up to normalizations and natural identifications, $\Psi=\Psi_{L} \circ \Psi_{C}$ coincides with the map $\Phi \circ \phi$ obtained by applying the scheme of [16] to the dNLS equation.

Remark 6.1. In the terminology of [16], the system of the Floquet exponents $\pm \omega_{j}\left(I_{S}, 0\right)$, $j \in S^{\perp}$, is nonresonant-see for example [2] where the relevant properties of the dNLS frequencies are discussed.

\section{Restrictions of $\Psi$}

In this section we present results concerning potentials $\varphi \in H_{r}^{0}$ which are even, odd, or real valued. To describe them, introduce the operator $T: H^{0}(\mathbb{T}, \mathbb{C}) \rightarrow H^{0}(\mathbb{T}, \mathbb{C})$ where for any $u \in H^{0}(\mathbb{T}, \mathbb{C}), T(u)$ is given by

$$
T(u)(x):=u(-x), \quad x \in \mathbb{R} \text { a.e. }
$$

(Here and in the sequel we identify an element in $H^{0}(\mathbb{T}, \mathbb{C})$ with a representative $f: \mathbb{R} \rightarrow \mathbb{C}$ of its lift, obtained by extending $f:[0,1) \rightarrow \mathbb{C}$ periodically in $x$ to $\mathbb{R}, f(x+n)=f(x), n \in \mathbb{Z}$. If this element is in $H^{s}(\mathbb{T}, \mathbb{C})$ with $s \in \mathbb{Z}_{\geq 1}$, then $f$ will be chosen to be the representative 
of period 1 which is in $\mathcal{C}^{s-1}(\mathbb{R}, \mathbb{C})$.) Let $T_{1}, T_{2}, T_{3}: H_{c}^{s}(\mathbb{T}, \mathbb{C}) \rightarrow H_{c}^{s}(\mathbb{T}, \mathbb{C}), s \in \mathbb{Z}_{s \geq 0}$, denote the involutions,

$$
T_{1}(u, v):=(T(u), T(v)), \quad T_{2}(u, v):=-(T(u), T(v)), \quad T_{3}(u, v):=(v, u)
$$

and $H_{r, j}^{s}$ the following subspaces of $H_{r}^{s}$,

$$
\begin{gathered}
H_{r, 1}^{s}:=\left\{(u, \bar{u}) \in H_{r}^{s}: T(u)=u\right\}, \\
H_{r, 2}^{s}:=\left\{(u, \bar{u}) \in H_{r}^{s}: T(u)=-u\right\},
\end{gathered}
$$

and

$$
H_{r, 3}^{s}:=\left\{(u, \bar{u}) \in H_{r}^{s}: u \text { real valued }\right\}
$$

For any $1 \leq j \leq 3$ and $s \in \mathbb{Z}_{s \geq 0}, T_{j}(u, v)=(u, v)$ on $H_{r, j}^{s}$. It is straightforward to verify that $\mathcal{H}^{\text {nls }}$ is left invariant by $T_{j}$,

$$
\mathcal{H}^{\mathrm{nls}}\left(T_{j} u\right)=\mathcal{H}^{\mathrm{nls}}(u) \quad \forall u \in H_{r}^{1}, 1 \leq j \leq 3,
$$

$T_{1}, T_{2}$ are canonical, and hence the subspaces $H_{r, 1}^{s}, H_{r, 2}^{s}$ are symplectic. In contrast, $T_{3}$ is not canonical and the subspace $H_{r, 3}^{s}$ Lagrangian. To describe how the involutions $T_{j}$ act on Birkhoff coordinates, we define the operator $\tilde{T}: h_{\mathbb{C}}^{0} \rightarrow h_{\mathbb{C}}^{0}$, defined for $x=\left(x_{k}\right)_{k \in \mathbb{Z}} \in h_{\mathbb{C}}^{0}$ by $(\tilde{T} x)_{k}:=x_{-k}, k \in \mathbb{Z}$, and introduce the involutions $\tilde{T}_{j}$ on $h_{r}^{s}, s \in \mathbb{Z}_{\geq 0}$, given by

$$
\tilde{T}_{1}(x, y):=(\tilde{T}(x), \tilde{T}(y)), \quad \tilde{T}_{2}(x, y):=-(\tilde{T}(x), \tilde{T}(y)), \quad \tilde{T}_{3}(x, y):=(\tilde{T}(x),-\tilde{T}(y))
$$

as well as the subspaces $h_{r, j}^{s}$ of $h_{r}^{s}$, defined by

$$
h_{r, j}^{s}:=\left\{(x, y) \in h_{r}^{s}: \tilde{T}_{j}(x, y)=(x, y)\right\}
$$

For any $1 \leq j \leq 3$ and $s \in \mathbb{Z}_{\geq 0}$, it follows from Theorem 1.2 in [8] that $\Phi^{\text {nls }} \circ T_{j}=\tilde{T}_{j} \circ \Phi^{\text {nls }}$ on $H_{r}^{s}$ implying that on $h_{r}^{s}$,

$$
\Psi^{\mathrm{nls}} \circ \tilde{T}_{j}=T_{j} \circ \Psi^{\mathrm{nls}} .
$$

Since elements in the subspaces $H_{r, j}^{s}$ and $h_{r, j}^{s}$ are kept fixed by the corresponding involutions introduced above, one then concludes from Theorem 1.1 that for any such $j$ and $s$, $\Psi^{\mathrm{nls}}: h_{r, j}^{s} \rightarrow H_{r, j}^{s}$ is a real analytic diffeomorphism. Furthermore, by (7.1) and (7.2), and 
the fact that $I\left(\tilde{T}_{j}(z)\right)=\tilde{T}(I(z))$ for any $1 \leq j \leq 3$, it follows that $H^{\text {nls }}(\tilde{T}(I))=H^{\text {nls }}(I)$ for any $I \equiv I(z)=\left(\left(x_{k}^{2}+y_{k}^{2}\right) / 2\right)_{k \in \mathbb{Z}}$ with $z=\left(\left(x_{k}\right)_{k \in \mathbb{Z},}\left(y_{k}\right)_{k \in \mathbb{Z}}\right) \in h_{r}^{1}$. Thus for any $k \in \mathbb{Z}$ ，

$$
\omega_{k}^{\mathrm{nls}}(\tilde{T}(I))=\left(\partial_{I_{k}} H^{\mathrm{nls}}\right)(\tilde{T}(I))=\partial_{I_{-k}}\left(H^{\mathrm{nls}}(\tilde{T}(I))\right)=\partial_{I_{-k}}\left(H^{\mathrm{nls}}(I)\right)=\omega_{-k}(I)
$$

In particular, $h_{r, 1}^{s}$ and $h_{r, 2}^{s}$ are left invariant by the dNLS flow (in Birkhoff coordinates). We remark that $h_{r, 3}^{s}$ is left invariant by the flow of the defocusing mKdV equation (cf. e.g., [13]). Furthermore note that for any $u \in H^{0}(\mathbb{T}, \mathbb{C})$, the Fourier coefficients $(T(u))_{n}$, $n \in \mathbb{Z}$, of $T(u)$ satisfy

$$
(T(u))_{n}=u_{-n}=\left(\tilde{T}\left(\left(u_{k}\right)_{k \in \mathbb{Z}}\right)\right)_{n} .
$$

The following proposition will be applied in subsequent work:

Proposition 7.1. In addition to the setup of Theorem 1.2, assume that $S \subset \mathbb{Z}$ is symmetric, $S=-S$, and $1 \leq j \leq 3$ and that the complex neighbourhood $\mathcal{V} \subset h_{c}^{0}$ of Theorem 1.2 is invariant with respect to $\tilde{T}_{j}, \tilde{T}_{j}(\mathcal{V})=\mathcal{V}$. Then for any $s \in \mathbb{Z}_{\geq 0}$ ，

$$
\Psi \circ \tilde{T}_{j}=T_{j} \circ \Psi \quad \text { on } \mathcal{V} \cap h_{r}^{s}
$$

As a consequence $\Psi: \mathcal{V} \cap h_{r, j}^{s} \rightarrow H_{r, j}^{s}$ is a real analytic diffeomorphism on to its image. Furthermore, on $\mathcal{V} \cap h_{r}^{1}$, the Hamiltonian $\mathcal{H}=\mathcal{H}^{\text {nls }} \circ \Psi$ is invariant under $\tilde{T}_{j}, \mathcal{H} \circ \tilde{T}_{j}=\mathcal{H}$, and in the expansion (1.6),

$$
\mathcal{H}(z)=H^{\mathrm{nls}}\left(I_{S}, 0\right)+\sum_{n \in S^{\perp}} \omega_{n}^{\mathrm{nls}}\left(I_{S}, 0\right) I_{n}(z)+\mathcal{P}_{3}(z)
$$

the three terms on the right-hand side, when restricted to $\mathcal{V} \cap h_{r}^{1}$, are in view of (7.3) invariant under $\tilde{T}_{j}$. Since in the case $1 \leq j \leq 2, \tilde{T}_{j}$ is canonical, it then follows that the Hamiltonian vector fields $X_{\mathcal{H}}$ and $X_{\mathcal{P}_{3}}$ (cf. (2.6)) satisfy on $\mathcal{V} \cap h_{r}^{1}$ and for $1 \leq j \leq 2$

$$
X_{\mathcal{H}}\left(\tilde{T}_{j}(z)\right)=\tilde{T}_{j} X_{\mathcal{H}}(z), \quad X_{\mathcal{P}_{3}}\left(\tilde{T}_{j}(z)\right)=\tilde{T}_{j} X_{\mathcal{P}_{3}}(z) .
$$

It implies that for $s \in \mathbb{Z}_{\geq 1}$,

$$
X_{\mathcal{H}}: \mathcal{V} \cap h_{r, j}^{s+2} \rightarrow h_{r, j}^{s}, \quad X_{\mathcal{P}_{3}}: \mathcal{V} \cap h_{r, j}^{s} \rightarrow h_{r, j}^{s}
$$

Proof. Since the proofs for $j=1,2$, and 3 are similar, we concentrate on the case $j=1$ only. Recall that $\Psi=\Psi_{L} \circ \Psi_{C}$ where the maps $\Psi_{L}$ and $\Psi_{C}$ were introduced in Sections 
3 and 4, respectively. In the latter section, the neighbourhood $\mathcal{V}$ in the statement of Theorem 1.2 is actually denoted by $\mathcal{V}_{\delta}^{\prime}$ (cf. Section 5.3). By (4.16), $\mathcal{V}_{\delta}^{\prime}$ is contained in the neighbourhood $\mathcal{V}$, which was introduced in Section 3. In the course of this proof we use the notation established in these two sections.

By (1.8), $\Psi_{L}\left(z_{S}, z_{\perp}\right)=\Psi^{\text {nls }}\left(z_{S}, 0\right)+\mathrm{d} \Psi^{\text {nls }}\left(z_{S}, 0\right)\left[0, z_{\perp}\right]$ for any $z=\left(z_{S}, z_{\perp}\right)$ in $\mathcal{V}$. Since $S=-S, T_{1}(\mathcal{V})=\mathcal{V},(7.2)$ applies to $\Psi^{\text {nls }}\left(z_{S}, 0\right)$ and $\mathrm{d} \Psi^{\text {nls }}\left(z_{S}, 0\right)\left[0, z_{\perp}\right]$ and hence

$$
T_{1} \circ \Psi_{L}=\Psi_{L} \circ \tilde{T}_{1}
$$

Next we show that $\tilde{T}_{1} \circ \Psi_{C}=\Psi_{C} \circ \tilde{T}_{1}$. Recall that $\Psi_{C}$, defined on $\mathcal{V}_{\delta}^{\prime} \cap h_{r}^{0}$ by (4.28), is given by the time-one flow of the non-autonomous vector field $X(z, \tau)=-\mathcal{L}_{\tau}(z)^{-1} E(z)$, introduced in (4.12). Here $0 \leq \tau \leq 1$ and for any $z \in \mathcal{V} \cap h_{r}^{0}$, the operators $\mathcal{L}_{\tau}(z)=$ $J^{-1}+\tau L(z): \mathbb{C}^{S} \times \mathbb{C}^{S} \times h_{\perp c}^{0} \rightarrow \mathbb{C}^{S} \times \mathbb{C}^{S} \times h_{\perp c}^{0}$ and $L(z): \mathbb{C}^{S} \times \mathbb{C}^{S} \times h_{\perp c}^{0} \rightarrow \mathbb{C}^{S} \times \mathbb{C}^{S} \times h_{\perp c}^{0}$ are defined in (4.5), respectively (3.39), and the element $E(z)=\left(E_{S}(z), 0\right) \in \mathbb{C}^{S} \times \mathbb{C}^{S} \times h_{\perp c}^{0}$ in (4.9) and (4.10). In a first step we prove that

$$
L\left(\tilde{T}_{1} z\right) \circ \tilde{T}_{1}=\tilde{T}_{1} \circ L(z) \quad \forall z \in \mathcal{V} \cap h_{r}^{0}
$$

Recall from (3.40) that $L(z)$ is of the form

$$
L(z)=\left(\begin{array}{cc}
L_{S}^{S}(z) & L_{S}^{\perp}(z) \\
L_{\perp}^{S}(z) & 0
\end{array}\right)
$$

where the operators $L_{S}^{S}(z), L_{S}^{\perp}(z)$, and $L_{\perp}^{S}(z)$ are defined in (3.41), (3.42), and (3.43), respectively. It is to show that for any $z=\left(z_{S}, z_{\perp}\right) \in \mathcal{V} \cap h_{r}^{0}$ and $\widehat{z}=\left(\widehat{z}_{S}, \widehat{z}_{\perp}\right)$ in $h_{r}^{0}, L_{S}^{S}(z)\left[\widehat{z}_{S}\right]$, $L_{S}^{\perp}(z)\left[\widehat{z}_{\perp}\right]$, and $L_{\perp}^{S}(z)\left[\widehat{z}_{S}\right]$ satisfy the symmetry conditions required for $L\left(\tilde{T}_{1} z\right)\left[\tilde{T}_{1} \widehat{z}\right]=$ $\tilde{T}_{1}(L(z)[\widehat{z}])$ to hold. Since the arguments for each of the vectors $L_{S}^{S}(z)\left[\widehat{z}_{S}\right], L_{S}^{\perp}(z)\left[\widehat{z}_{\perp}\right]$, and $L_{\perp}^{S}(z)\left[\widehat{z}_{S}\right]$ are similar, we consider only $L_{\perp}^{S}(z)\left[\widehat{z}_{S}\right]$. By $(3.43)$, it is given by

$$
L_{\perp}^{S}(z)\left[\widehat{z}_{S}\right]=\mathrm{i}\left(\begin{array}{l}
\left(\left\langle\mathbb{J} \mathrm{d}_{S}\left(\mathrm{~d}_{\perp} \Psi^{\mathrm{nls}}\left(\Pi_{S} z\right)\left[z_{\perp}\right]\right)\left[\widehat{z}_{S}\right], \partial_{x_{j}} \Psi^{\mathrm{nls}}\left(\Pi_{S} z\right)\right\rangle_{r}\right)_{j \in S^{\perp}} \\
\left(\left\langle\mathbb{J} \mathrm{d}_{S}\left(\mathrm{~d}_{\perp} \Psi^{\mathrm{nls}}\left(\Pi_{S} z\right)\left[z_{\perp}\right]\right)\left[\widehat{z}_{S}\right], \partial_{Y_{j}} \Psi^{\mathrm{nls}}\left(\Pi_{S} z\right)\right\rangle_{r}\right)_{j \in S^{\perp}}
\end{array}\right)
$$

The two components of $L_{\perp}^{S}(z)\left[\widehat{z}_{S}\right]$ can be analysed in the same way, so it suffices to look at the first one. Write $z=(x, y)$. Further introduce $G^{(1)}(x, y)\left[\widehat{z}_{S}\right]:=\mathbb{J} \mathrm{d}_{S}\left(\mathrm{~d}_{\perp} \Psi^{\text {nls }}\left(\Pi_{S} z\right)\left[z_{\perp}\right]\right)\left[\widehat{z}_{S}\right]$ and $G^{(2)}(x, y):=\Psi^{\mathrm{nls}}\left(\Pi_{S} z\right)$, which are both elements in $H_{r}^{0}$, and define for any $j \in S^{\perp}$

$$
a_{j}:=\left\langle G^{(1)}(x, y), \partial_{x_{j}} G^{(2)}(x, y)\right\rangle_{r}, \quad b_{j}:=\left\langle G^{(1)}\left(\tilde{T}_{1}(x, y)\right)\left[\tilde{T}_{1} \widehat{z}_{S}\right],\left(\partial_{x_{j}} G^{(2)}\right)\left(\tilde{T}_{1}(x, y)\right)\right\rangle_{r} .
$$


We show that $b_{j}=a_{-j}$ for any $j \in S^{\perp}$. Indeed, by [8] one has

$$
G^{(1)}\left(\tilde{T}_{1}(x, y)\right)\left[\tilde{T}_{1} \widehat{z}_{S}\right]=T_{1}\left(G^{(1)}(x, y)\left[\widehat{z}_{S}\right]\right), \quad G^{(2)}\left(\tilde{T}_{1}(x, y)\right)=T_{1}\left(G^{(2)}(x, y)\right) .
$$

Apply $\partial_{x_{j}}$ to both sides of the latter identity to get

$$
\left(\partial_{x_{j}} G^{(2)}\right)\left(\tilde{T}_{1}(x, y)\right)=\partial_{x_{-j}}\left(G^{(2)}\left(\tilde{T}_{1}(x, y)\right)\right)=\partial_{x_{-j}} T_{1}\left(G^{(2)}(x, y)\right)=T_{1}\left(\partial_{x_{-j}} G^{(2)}(x, y)\right) .
$$

When combined with (7.8), one then concludes that for any $j \in S^{\perp}$,

$$
b_{j}=\left\langle T_{1}\left(G^{(1)}(x, y)\left[\widehat{z}_{S}\right]\right), T_{1}\left(\partial_{x_{-j}} G^{(2)}(x, y)\right)\right\rangle_{r}=\left\langle G^{(1)}(x, y)\left[\widehat{z}_{S}\right], \partial_{x_{-j}} G^{(2)}(x, y)\right\rangle_{r}=a_{-j} .
$$

We thus have verified (7.7). Since $\mathcal{L}_{\tau}(z)=J^{-1}+\tau L(z), 0 \leq \tau \leq 1$, with $J^{-1}=\left(\begin{array}{cc}0 & \operatorname{Id} \\ -\operatorname{Id} & 0\end{array}\right)$, $\mathcal{L}_{\tau}(z)$ is invertible (cf. Lemma 4.1), $\tilde{T}_{1}^{-1}=\tilde{T}_{1}$, and $\tilde{T}_{1}\left(\mathcal{V} \cap h_{r}^{0}\right)=\mathcal{V} \cap h_{r}^{0}$ (by assumption), one then also has $\mathcal{L}_{\tau}^{-1}\left(\tilde{T}_{1} z\right) \circ \tilde{T}_{1}=\tilde{T}_{1} \circ \mathcal{L}_{\tau}^{-1}(z)$ for any $z$ in $\mathcal{V} \cap h_{r}^{0}$. Furthermore, $E(z)=\left(\frac{1}{2} L_{S}^{\perp}(z)\left[z_{\perp}\right], 0\right)$ (cf. (4.10)) satisfies $E\left(\tilde{T}_{1} z\right)=\tilde{T}_{1} E(z)$. Altogether we conclude that the vectorfield $X(z, \tau)=-\mathcal{L}_{\tau}(z)^{-1} E(z)$ (cf. (4.12)) has the property that for any $z \in \mathcal{V} \cap h_{r}^{0}$ and $0 \leq \tau \leq 1, X\left(\tilde{T}_{1} z, \tau\right)=\tilde{T}_{1} X(z, \tau)$. Hence by Lemma 4.4, $\Psi_{C}$, given by the time-one flow of the vector field $X(z, \tau)$, satisfies $\tilde{T}_{1} \circ \Psi_{C}=\Psi_{C} \circ \tilde{T}_{1}$ on $\mathcal{V}_{\delta}^{\prime} \cap h_{r}^{0}$. When combined with the identity (7.6), we therefore have proved that $T_{1} \circ \Psi=\Psi \circ \tilde{T}_{1}$ on $\mathcal{V}_{\delta}^{\prime} \cap h_{r}^{0}$. Clearly, the corresponding identity also holds on $\mathcal{V}_{\delta}^{\prime} \cap h_{r}^{s}$ for any $s \in \mathbb{Z}_{\geq 1}$. Concerning (7.4), note that in view of the definition (2.6) of a Hamiltonian vector field,

$$
X_{\mathcal{H}}\left(\tilde{T}_{j}(z)\right)=J(\nabla H)\left(\tilde{T}_{j}(z)\right)=J \tilde{T}_{j} \nabla\left(H\left(\tilde{T}_{j}(z)\right)\right)=J \tilde{T}_{j}(\nabla H(z))=\tilde{T}_{j} J(\nabla H(z))=\tilde{T}_{j} X_{\mathcal{H}}(z)
$$

A similar computation shows that $X_{\mathcal{P}_{3}}\left(\tilde{T}_{j}(z)\right)=\tilde{T}_{j} X_{\mathcal{P}_{3}}(z)$. The remaining statements of the proposition are an immediate consequence of the proved identities.

\section{Funding}

This work was supported in part by the Swiss National Science Foundation.

\section{Appendix 1: A Version of the Poincaré Lemma}

We follow the general approach of [18, Chapter V], and restrict to the finite-dimensional setup as the extension to infinite dimension is straightforward by restriction, see 
[16, Lemma 1.1]. Let $E=\mathbb{R}^{n}$ and denote by $L_{a}^{r}(E)$ the space of multilinear continuous alternating forms of degree $0 \leq r \leq n$. Let $U \subseteq E$ be an open nonempty set and consider

$$
\omega: U \rightarrow L_{a}^{r}(E)
$$

For any $z \in U$, denote by

$$
\omega(z)\left[\xi_{1}, \ldots, \xi_{r}\right] \in \mathbb{R}
$$

the value of $\omega(z)$ when evaluated at $\xi_{1}, \ldots, \xi_{r} \in E$. Similarly, if $\xi_{j}=\xi_{j}(z) \in E, j=1, \ldots, r$, are vector fields on $U$, then we denote by $\omega\left[\xi_{1}, \ldots, \xi_{r}\right]$ the function

$$
U \rightarrow \mathbb{R}, \quad z \mapsto \omega(z)\left[\xi_{1}(z), \ldots, \xi_{r}(z)\right]
$$

Furthermore, we denote by $\omega^{\prime}(z) \cdot \xi, \xi \in E$, the alternating $r$-form

$$
\left.\partial_{\varepsilon}\right|_{\varepsilon=0} \omega(z+\varepsilon \xi) \in L_{a}^{r}(E)
$$

The exterior differential $\mathrm{d} \omega$ of $\omega$, evaluated at $z \in U, \xi_{1}, \ldots, \xi_{r+1} \in E$, is then given by the formula

$$
\sum_{j=1}^{r+1}(-1)^{j+1} \omega^{\prime}(z) \cdot \xi_{j}\left[\xi_{1}, \ldots, \xi_{j-1}, \xi_{j+1}, \ldots, \xi_{r+1}\right]
$$

also referred to as Cartan's formula. Let us now consider the case where

$$
\begin{gathered}
E=\mathbb{R}^{n_{1}} \times \mathbb{R}^{n_{2}}, \quad n=n_{1}+n_{2}, \quad n_{2} \geq 1, \\
U=U_{1} \times U_{2} \subseteq \mathbb{R}^{n_{1}} \times \mathbb{R}^{n_{2}},
\end{gathered}
$$

and $U_{2}$ is a ball in $\mathbb{R}^{n_{2}}$ centred at 0 . We denote the elements of $U$ by $z=(x, y)$ and the ones of $E$ by $\xi=(V, W) \in \mathbb{R}^{n_{1}} \times \mathbb{R}^{n_{2}}$. For any $r$-form $\omega$ on $U$, denote by $\omega_{\mathcal{C}}$ the $(r-1)$-form on $U$, obtained by the cone construction: for any $x \in U_{1}, y \in U_{2}, V_{1}, \ldots, v_{r-1} \in \mathbb{R}^{n_{1}}$, and $w_{1}, \ldots, w_{r-1} \in \mathbb{R}^{n_{2}}$,

$$
\omega_{\mathcal{C}}(x, y)\left[\left(v_{1}, w_{1}\right), \ldots,\left(v_{r-1}, w_{r-1}\right)\right]=\int_{0}^{1} \omega(x, t y)\left[(0, y),\left(v_{1}, t w_{1}\right), \ldots,\left(V_{r-1}, t w_{r-1}\right)\right] d t
$$

Since $U_{2}$ is a ball in $\mathbb{R}^{n_{2}}$, centred at 0 , for any $0 \leq t \leq 1,(x, t y)$ is in $U_{1} \times U_{2}$ and hence $\omega(x, t y)$ in (A.3) is well defined. 
Lemma A.1 (Poincaré lemma). Assume that $\omega$ is a $r$-form on $U=U_{1} \times U_{2}$, with $1 \leq r \leq n$ and $n_{2} \geq 1$, satisfying

$$
\omega(x, 0)\left[\left(v_{1}, 0\right), \ldots,\left(v_{r}, 0\right)\right]=0, \quad \forall x \in U_{1}, \quad \forall v_{1}, \ldots, v_{r} \in \mathbb{R}^{n_{1}}
$$

Then

$$
\mathrm{d}\left(\omega_{\mathcal{C}}\right)+(\mathrm{d} \omega)_{\mathcal{C}}=\omega
$$

In particular, if in addition $\omega$ is closed, $\mathrm{d} \omega=0$, then $\mathrm{d}\left(\omega_{\mathcal{C}}\right)=\omega$.

\section{Appendix 2: $\quad$ Formulas for $\mathrm{d} \Psi^{\mathrm{nls}}\left(z_{S}, 0\right)\left[\left(0, z_{\perp}\right)\right]$}

Note that for $z=\left(z_{S}, z_{\perp}\right)$ with $z_{S} \in \mathbb{R}^{S} \times \mathbb{R}^{S}$ and $z_{\perp}=\left(\left(x_{j}\right)_{j \in S^{\perp}},\left(y_{j}\right)_{j \in S^{\perp}}\right) \in h_{\perp r^{\prime}}^{0}$

$$
\mathrm{d} \Psi^{\mathrm{nls}}\left(z_{S}, 0\right)\left[\left(0, z_{\perp}\right)\right]=\sum_{j \in S^{\perp}} x_{j} \mathrm{~d} \Psi^{\mathrm{nls}}\left(z_{S}, 0\right)\left[\mathrm{e}^{(1, j)}\right]+\sum_{j \in S^{\perp}} y_{j} \mathrm{~d} \Psi^{\mathrm{nls}}\left(z_{S}, 0\right)\left[\mathrm{e}^{(2, j)}\right],
$$

where for any $j \in S^{\perp}$,

$$
\mathrm{e}^{(1, j)}=\left(\left(\delta_{n j}\right)_{n \in \mathbb{Z}}, 0\right), \quad \mathrm{e}^{(2, j)}=\left(0,\left(\delta_{n j}\right)_{n \in \mathbb{Z}}\right)
$$

It turns out that for $j \in S^{\perp}, \mathrm{d} \Psi^{\mathrm{nls}}\left(z_{S}, 0\right)\left[\mathrm{e}^{(1, j)}\right]$ and $\mathrm{d} \Psi^{\mathrm{nls}}\left(z_{S}, 0\right)\left[\mathrm{e}^{(2, j)}\right]$ can be computed quite explicitly. Consider the Hamiltonian equation with Hamiltonian given by the coordinate function $x_{j}, \partial_{t} w=\mathrm{i} \rrbracket \partial x_{j}$, and denote by $w(t)$ its solution with initial data $w(0)=\Psi^{\mathrm{nls}}\left(z_{S}, 0\right)$. Then $z(t):=\Phi^{\mathrm{nls}}(w(t))$ solves

$$
\partial_{t} z=\mathrm{d} \Phi^{\mathrm{nls}}(w(t)) \partial_{t} w(t)=\mathrm{d} \Phi^{\mathrm{nls}}(w(t)) \mathrm{i} \rrbracket \partial x_{j}
$$

Since by Theorem 1.1, $\Phi^{\text {nls }}$ is symplectic, one has $\partial_{t} z=J \mathrm{e}^{(1, j)}=\mathrm{e}^{(2, j)}$. When combined with (B.1) it implies that $\mathrm{d} \Psi^{\mathrm{nls}}(z(t))\left[\mathrm{e}^{(2, j)}\right]=\mathrm{i} \rrbracket \partial x_{j}$. Similarly, one derives the corresponding identity for the coordinate function $y_{j}$. When evaluated at $t=0$ we then obtain

$$
\mathrm{d} \Psi^{\mathrm{nls}}\left(z_{S}, 0\right)\left[\mathrm{e}^{(2, j)}\right]=\mathrm{i} \rrbracket \partial x_{j}=\left(-\mathrm{i} \partial_{V} x_{j}, \mathrm{i} \partial_{u} x_{j}\right), \quad \mathrm{d} \Psi^{\mathrm{nls}}\left(z_{S}, 0\right)\left[\mathrm{e}^{(1, j)}\right]=\mathrm{i} \rrbracket \partial y_{j}=\left(-\mathrm{i} \partial_{V} y_{j}, \mathrm{i} \partial_{u} y_{j}\right)
$$

By the definition of $x_{j}, y_{j}$ in [10, p. 113], one has for a potential $w \in H_{r}^{0}$ with Birkhoff coordinates $\left(z_{S}, 0\right)$ (referred to as $S$-gap potential)

$$
x_{j}=\frac{\xi_{j}}{\sqrt{8}}\left(\mathrm{e}^{\mathrm{i} \beta_{j}} \mathfrak{z}_{j}+\mathrm{e}^{-\mathrm{i} \beta_{j}} \mathfrak{z}_{j}\right), \quad y_{j}=\frac{\xi_{j}}{\sqrt{8} \mathrm{i}}\left(\mathrm{e}^{\mathrm{i} \beta_{j}} \mathfrak{z}_{j}-\mathrm{e}^{-\mathrm{i} \beta_{j}} \mathfrak{z}_{j}\right),
$$


where $\mathfrak{z}_{j}^{ \pm}=\gamma_{j} \mathrm{e}^{ \pm i \eta_{j}}$ if $\gamma_{j} \neq 0$ and $\mathfrak{z}_{j}^{ \pm}=0$ otherwise. We refer to [10] for the definitions of $\xi_{j}$, $\eta_{j}$, and $\beta_{j}$. Since $w$ is assumed to be a $S$-gap potential, it follows that for any $j \in S^{\perp}$,

$$
\partial x_{j}=\frac{\xi_{j}}{\sqrt{8}}\left(\mathrm{e}^{\mathrm{i} \beta_{j}} \partial \mathfrak{z}_{j}^{+}+\mathrm{e}^{-\mathrm{i} \beta_{j}} \partial \mathfrak{z}_{j}^{-}\right), \quad \partial y_{j}=\frac{\xi_{j}}{\sqrt{8} \mathrm{i}}\left(\mathrm{e}^{\mathrm{i} \beta_{j}} \partial \mathfrak{z}_{j}^{+}-\mathrm{e}^{-\mathrm{i} \beta_{j}} \partial \mathfrak{z}_{j}^{-}\right),
$$

where by formula (17.3) in [10],

$$
\partial \mathfrak{z}_{j}^{ \pm}=2\left(\partial \tau_{j}-\partial \mu_{j}\right) \pm\left(\mathrm{i} 2 \delta\left(\mu_{j}\right) \partial \phi_{j}+2 \phi_{j}\left(\left.\mathrm{i} \partial \delta\right|_{\lambda=\mu_{j}}+\mathrm{i} \dot{\delta}\left(\mu_{j}\right) \partial \mu_{j}\right)\right)
$$

We refer to [10] for the definitions of the various quantities as well as for formulas of the gradients in the latter expression. Each of the two components of these gradients are shown to be a linear combination of quadratic expressions in the entries of the fundamental solution $M=M(x, \lambda)$ of the Zakharov Shabat operator

$$
L:=\left(\begin{array}{cc}
\mathrm{i} & 0 \\
0 & -\mathrm{i}
\end{array}\right) \partial_{X}+\left(\begin{array}{cc}
0 & u \\
\bar{u} & 0
\end{array}\right), \quad w=(u, v)=(u, \bar{u})
$$

In fact, in [11], it has been proved that

$$
\partial \mathfrak{z}_{j}^{ \pm}=\left(\left(K_{j 2} \pm \mathrm{i} H_{j 2}\right)^{2},\left(K_{j 1} \pm \mathrm{i} H_{j 1}\right)^{2}\right)
$$

where

$$
H_{j}=\left(H_{j 1}, H_{j 2}\right)=\left.\frac{1}{\left\|M_{1}+M_{2}\right\|_{L^{2}}}\left(M_{1}+M_{2}\right)\right|_{\lambda=\mu_{j}}
$$

denotes the $L^{2}$-normalized eigenfunction of $L$ for the Dirichlet eigenvalue $\mu_{j}, M_{1}, M_{2}$ are the two columns of $M$, and $K_{j}=\left(K_{j 1}, K_{j 2}\right)$ is the $L^{2}$-normalized solution of $L F=$ $\mu_{j} F$, which is $L^{2}$-orthogonal to $H_{j}$ and satisfies the additional normalization condition $-\mathrm{i}\left(K_{j 1}(0)-K_{j 2}(0)\right)>0$.

\section{References}

[1] Baldi, P., M. Berti, and R. Montalto. "KAM for autonomous quasi-linear perturbations of KdV." Annales de l'Institut H. Poincaré (C) Analyse Non Linéaire. doi:10.1016/j.anihpc.2015.07.003.

[2] Berti, M., T. Kappeler, and R. Montalto. “Large KAM tori for semilinear perturbations of the defocusing NLS equation." (2016), arXiv:1603.09252.

[3] Berti, M., and R. Montalto. "Quasi-periodic standing wave solutions of gravity capillary standing water waves." (2016), arXiv:1602.02411. 
[4] Bourgain, J. "Nonlinear Schrödinger equations." In Hyperbolic Equations and Frequency Interactions, edited by L. Cafferelli and E. Weinan. IAS/Park City Mathematics Series, Providence, American Mathematical Society, 1999.

[5] Craig, W., and C. Wayne. "Periodic solutions of nonlinear Schrödinger equations and Nash Moser method" In Hamiltonian Mechanics, Toruñ, 1993, edited by J. Seimenis, NATO Advanced Science Institutes Series B Physics, 331, 103-22, New York, Plenum Press, 1994.

[6] Geng, J., and J. You. "A KAM theorem for the one dimensional Schrödinger equation with periodic boundary conditions." Journal of Differential Equations 209 (2005): 1-56.

[7] Geng, J., and J. You. "A KAM theorem for Hamiltonian partial differential equations in higher dimensional spaces." Communications in Mathematical Physics 262 (2006): 343-72.

[8] Grébert, B., and T. Kappeler. "Symmetries of the nonlinear Schrödinger equation." Bulletin de la Société Mathématique de France 130, no. 4 (2002): 603-18.

[9] Grébert, B., and T. Kappeler. "Perturbations of the defocusing nonlinear Schrödinger equation." Milan Journal of Mathematics 71 (2003): 141-74.

[10] Grébert, B., and T. Kappeler. The Defocusing NLS Equation and Its Normal Form. Zürich, European Mathematical Society, 2014.

[11] Grébert, B., T. Kappeler, and J. Pöschel. "Normal form theory for the NLS equation: a preliminary report." Unpublished notes, 2003.

[12] Kappeler, T., and Z. Liang. "A KAM theorem for the defocusing NLS equation." Journal of Differential Equations 252, no. 6 (2012): 4068-113.

[13] Kappeler, T., and J. Molnar. On the wellposedness of the defocusing $m K d V$ equation below $L^{2},(2016)$, arXiv: 1606.07052.

[14] Kappeler, T., B. Schaad, and P. Topalov. "Semi-linearity of the nonlinear Fourier transform of the defocusing NLS equation." International Mathematics Research Notices (2016), doi: 10.1093/imrn/rnv397.

[15] Krichever, I. "Perturbation theory in periodic problems for two-dimensional integrable systems." Soviet Scientific Reviews. Section C, Mathematical Physics 9 (1991): 1-103.

[16] Kuksin, S. Analysis of Hamiltonian PDEs, Oxford, Oxford University Press, 2000.

[17] Kuksin, S., and J. Pöschel, "Invariant Cantor manifolds of quasi-periodic oscillations for a nonlinear Schrödinger equation." Annals of Mathematics 143 (1996): 149-79.

[18] Lang, S. Differentiable Manifolds. Addison-Wesley, 1972.

[19] Molnar, J. "New estimates of the nonlinear Fourier transform for the defocusing NLS equation." International Mathematics Research Notices 2015, no. 17 (2015): 8309-52. 